\title{
Link Adaptation for Mitigating Earth-to-Space Propagation Effects on the NASA SCaN Testbed
}

\author{
Deirdre Kathleen Kilcoyne \\ Thesis submitted to the Faculty of the \\ Virginia Polytechnic Institute and State University \\ in partial fulfillment of the requirements for the degree of \\ Master of Science \\ in \\ Electrical Engineering \\ Robert W. McGwier, Chair \\ R. Michael Buehrer \\ A. A. (Louis) Beex \\ William C. Headley \\ April 20, 2016 \\ Blacksburg, Virginia
}

Keywords: link adaptation, adaptive modulation, shadowing, error vector magnitude 


\title{
Link Adaptation for Mitigating Earth-to-Space Propagation Effects on the NASA SCaN Testbed
}

\author{
Deirdre Kathleen Kilcoyne
}

\section{Academic Abstract}

In Earth-to-Space communications, well-known propagation effects such as path loss and atmospheric loss can lead to fluctuations in the strength of the communications link between a satellite and its ground station. Additionally, a less-often considered effect of shadowing due to the geometry of the satellite and its solar panels can also lead to link degradation. As a result of these anticipated channel impairments, NASA communication links have been traditionally designed to handle the worst-case impact of these effects through high link margins and static, lower rate, modulation formats. This thesis first characterizes the propagation environment experienced by a software-defined radio on the NASA SCaN Testbed through a full link-budget analysis. Then, the following chapters propose, design, and model a link adaptation algorithm to provide an improved trade-off between data rate and link margin through varying the modulation format as the received signal-to-noise ratio fluctuates. 


\title{
Link Adaptation for Mitigating Earth-to-Space Propagation Effects on the NASA SCaN Testbed
}

\author{
Deirdre Kathleen Kilcoyne
}

\section{Public Abstract}

As communications signals travel through space, they experience degradation and can encounter obstacles that decrease the signal strength, leaving the receiving party with poor links. The NASA SCaN Testbed on-board the International Space Station (ISS) is one such communications hub that experiences fluctuating signal strength, caused by solar panel obstruction. The ISS solar panels occasionally block the signals from reaching the receiving antenna, causing a blackout or many errors in the received data. To counteract the signal loss, NASA communication links currently operate at the most robust transmitting configuration, ensuring a low error-rate. However, with this worst-case method, the trade-off manifests in the very constrained data rate-although the data is transmitted with minimal errors, the throughput also remains low. This thesis first outlines the propagation environment experienced by a communications signal originating in Cleveland, $\mathrm{OH}$ and traveling to a software-defined radio on the NASA SCaN Testbed. Then, a link adaptation algorithm is designed and simulated to provide an improved trade-off between data rate and bit error rate. This flexibility between transmitting configurations allows for communications to be more efficient, as the transmitter can adapt as the signal strength improves. 


\section{Acknowledgments}

My journey to this point has only been made possible through my extraordinary support group. My thanks, gratitude, and love goes to y'all. Most importantly, to my mother who from the very beginning expected me to do my best at all times and who originally inspired me to be an engineer. To my advisor and fearless leader, Dr. Robert W. McGwier, for opening up his lab and life to me. To Chris Headley, "Chead", for guiding me as I grew from a small, baby sophomore electronics student to a still-small, adolescent Master's student. To Joey Ernst, for constantly brow-beating me for down-playing my technical capabilities; the tough-love was (and is) needed. To Francis Nguyen, my right-hand man, for the many hours spent together in class, lab, dining halls, and homework parties. To all of the Humens, for embracing me as I am and providing the best graduate lab community a gal could ask for. My most heartfelt thanks and appreciation go to the NASA SCaN Testbed team for our bi-weekly meetings and their patient support. And one final thanks to my mom again, because, honestly, who would I be without her? 


\section{Contents}

1 Introduction $\quad 1$

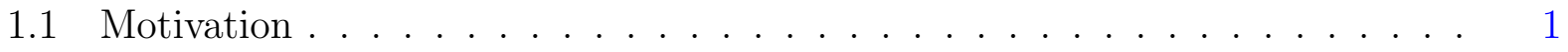

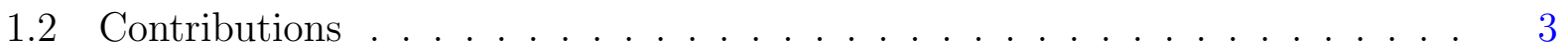

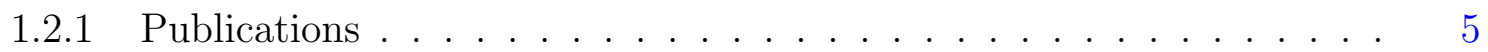

2 System Configuration $\quad 6$

2.1 SCaN Testbed Overview . . . . . . . . . . . . . . . . 6

2.1.1 On-board JPL SDR . . . . . . . . ........ 8

2.2 Glenn Research Center Ground Station . . . . . . . . . . . . . . . 8

2.3 Communications Link Configuration _. . . . . . . . . . . . . . . . . 9

2.3.1 Experimental Path Uplink . . . . . . . . . . . . . . . 10

2.3.2 Decision Logic . . . . . . . . . . . . . . . . 11

2.3.3 Experimental Path Downlink . . . . . . . . . . . . 12

3 Propagation Analysis $\quad 14$

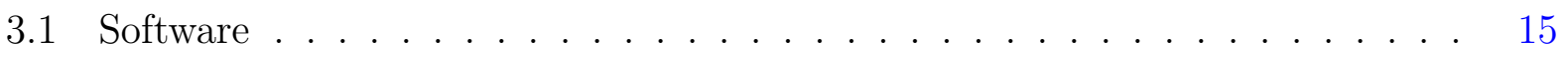

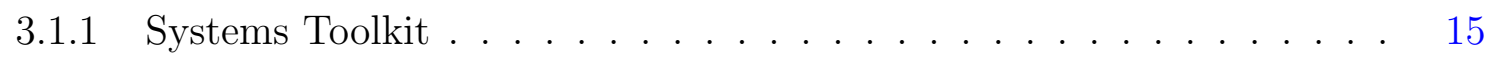

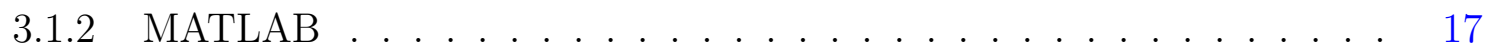

3.2 Path Loss . . . . . . . . . . . . . . . . . . . . . . 18

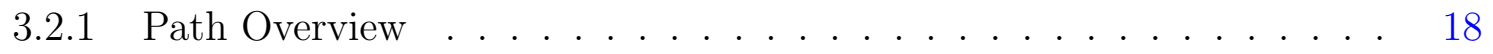

3.2.2 Path Loss Calculation . . . . . . . . . . . . . . . 20

3.3 Shadowing . . . . . . . . . . . . . . . . . . . 22 
3.3 .1 Obscuration Tool . . . . . . . . . . . . . . . . . . . . . . . 23

3.3 .2 Shadowing Loss . . . . . . . . . . . . . . . . . . . . . . . 25

3.4 System Parameters . . . . . . . . . . . . . . . . . . . . . . . . . 26

3.4.1 Transmitter Antenna Gain . . . . . . . . . . . . . . . . . . . . 27

3.4 .2 Receiver Antenna Gain . . . . . . . . . . . . . . . . . . . . 27

3.4 .3 Receiver Noise Temperature . . . . . . . . . . . . . . . . . . . . 28

3.4 .4 System Noise Temperature . . . . . . . . . . . . . . . . . . . . . . 29

3.5 Link Budget . . . . . . . . . . . . . . . . . . . . . . . . . . . . . . . . . . 29

3.5.1 Received Power . . . . . . . . . . . . . . . . . . . 30

3.5.2 Carrier-to-Noise Ratio . . . . . . . . . . . . . . . . . . . . . 31

3.5.3 Best-Case Link Budget . . . . . . . . . . . . . . . . . . . . . 32

3.5.4 Worst-Case Link Budget . . . . . . . . . . . . . . . . . . . . 33

3.6 Doppler Shift . . . . . . . . . . . . . . . . . . . . . . . . . 34

$\begin{array}{lll}4 & \text { Link Adaptation Algorithm } & 37\end{array}$

4.1 Prior Work . . . . . . . . . . . . . . . . . . . . . . . . . . 37

4.2 Decision Metric . . . . . . . . . . . . . . . . . . . 41

4.2 .1 Bit Error Rate . . . . . . . . . . . . . . . . . . . . . . . . . . 42

4.2 .2 Signal-to-Noise Ratio . . . . . . . . . . . . . . . . . . . . . . . 44

4.2 .3 SNR Measurement . . . . . . . . . . . . . . . . . . . 50

4.2 .4 Error Vector Magnitude . . . . . . . . . . . . . . . . . . . 51

4.3 Performance Analysis . . . . . . . . . . . . . . . . . . . . . . . . . 61

5 System Simulation $\quad 65$

6 Current and Future Work $\quad 75$

6.1 Flight Experiment . . . . . . . . . . . . . . . . . 75

6.1 .1 Xilinx System Generator . . . . . . . . . . . . . . . . . 76

6.1 .2 Clock Rate Discussion . . . . . . . . . . . . . . . . . . 76

$6.1 .3 \quad$ FPGA Transmitter . . . . . . . . . . . . . . . . . 76 
6.1.3.1 Matched Filter . . . . . . . . . . . . . . . . 77

$6.1 .4 \quad$ FPGA Receiver . . . . . . . . . . . . . . . . . . . . 80

6.1 .5 GPP Algorithm . . . . . . . . . . . . . . . . . . . . 82

6.2 Future Work . . . . . . . . . . . . . . . . . . . . . . 84

$\begin{array}{llr}7 & \text { Conclusions } & 85\end{array}$

$\begin{array}{ll}\text { Appendix A } & 87\end{array}$

$1.1 \quad$ TLE and SGP $4 \ldots \ldots \ldots \ldots \ldots \ldots \ldots$

1.2 Propagation Modeling Complete Table of Values . . . . . . . . . . . . . 87

1.3 Gray Coded Modulation Schemes . . . . . . . . . . . . . . . . . . . 89

$\begin{array}{ll}\text { Bibliography } & 92\end{array}$ 


\section{List of Figures}

2.1 Position of NASA SCAN Testbed on-board the ISS ELC. . . . . . . . . 7

2.2 S-band ground station antenna at GRC, $2.4 \mathrm{~m}$ dish. . . . . . . . . . . . 9

2.3 System configuration of communications link. . . . . . . . . . . 10

3.1 Flight antenna obstructed by solar panels. . . . . . . . . . . . . . . . 16

3.2 Simulated ground track, path loss, \& elevation for shortest \& longest accesses. 19

3.3 Time-varying path loss for 60 accesses. . . . . . . . . . . . . . . . . . 21

3.4 STK obscuration tool showing stages of a solar panel rotation. . . . . . . . . 23

3.5 Histogram of percentage area-obscured from STK obscuration tool. . . . . . 24

3.6 Shadowing loss curves for all obstructed accesses. . . . . . . . . . . . . 26

3.7 STK RX antenna for gain estimation. . . . . . . . . . . . 28

3.8 RX antenna gain matrix. . . . . . . . . . . . . . . . . . . . . 28

3.9 Ground track of best-case link budget. . . . . . . . . . . . . . . . . 32

3.10 Ground track of worst-case link budget. . . . . . . . . . . . . . . . . 34

3.11 Time-varying Doppler shift for 60 accesses. . . . . . . . . . . . . . . . 36

4.1 Bit error rate curves for analyzed modulation schemes. . . . . . . . . . . . 46

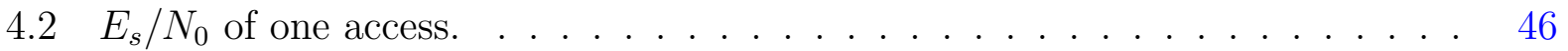

4.3 BER calculations for one access. . . . . . . . . . . . . . . . . 46

4.4 Switching thresholds for SNR. . . . . . . . . . . . . . . . . . . . . 49

4.5 Example of QPSK EVM measurement-closest symbol. . . . . . . . . . . . 52

4.6 Comparison of EVM with and without reference symbols. . . . . . . . . . 53 
4.7 Standard deviation of EVM measurements as SNR increases. . . . . . . . . . 54

4.8 Mean-squared error of EVM-estimated SNR and true SNR for varying N. . . 55

4.9 Linear relationship between EVM-estimated SNR and true SNR. . . . . . . 56

4.10 Comparison of theory BER and EVM-estimated BER for varying $N$. . . . 57

4.11 BER $_{\text {EVM }}$ and BER theory vs. SNR. . . . . . . . . . . . . . . 57

$4.12 \mathrm{BER}_{\mathrm{EVM}}$ vs. EVM. . . . . . . . . . . . . . . . . 57

4.13 Demonstration of QPSK/BPSK EVM thresholds with hysteresis. . . . . . . 60

4.14 BER of LA Algorithms varying across time. . . . . . . . . . . . . . . 62

4.15 Modulation schemes of LA algorithms varying across time. . . . . . . . . 63

4.16 Total number of transmitted bits of each LA algorithm varying across time. . 64

5.1 Flowgraph for the system simulation stages. . . . . . . . . . . . . 66

5.2 Spectral efficiency of LA algorithm compared to fixed-modulation. . . . . . . 68

5.3 Switching scenario with all 5 modes. . . . . . . . . . . . . . 70

5.4 Data throughput for scenario in Fig. 5.3. . . . . . . . . . . . . . 70

5.5 Access with severe shadowing. . . . . . . . . . . . . . 71

5.6 Data throughput for scenario in Fig. 5.5. . . . . . . . . . . . . . 71

5.7 Access that shows jumping modes. . . . . . . . . . . . . . 73

5.8 Data throughput for scenario in Fig. 5.7. . . . . . . . . . . . . 73

5.9 Shadowing and jump in mode. . . . . . . . . . . . . . . . 74

5.10 Data throughput for scenario in Fig. 5.9. . . . . . . . . . . . . . 74

6.1 Time domain response of the root-raised cosine filter coefficients. . . . . . . 77

6.2 System generator transmitter design flowgraph. . . . . . . . . . . . . 78

6.3 "Received" symbols from designed transmitter after matched filtering. . . . . 79

6.4 Comparison of transmitted and received bit values. . . . . . . . . . . . 80

6.5 System Generator receiver design flowgraph. . . . . . . . . . . . . . 81

6.6 DDS-introduced error on received symbols after matched-filtering. . . . . . . 83

A.1 BPSK Gray Code Arrangement. . . . . . . . . . . . . . . . . . . . 89 
A.2 QPSK Gray Code Arrangement. . . . . . . . . . . . . . . . . . 90

A.3 8PSK Gray Code Arrangement. . . . . . . . . . . . . . . . . . . . 90

A.4 16QAM Gray Code Arrangement. . . . . . . . . . . . . . . . . . . . . . 91

A.5 64QAM Gray Code Arrangement. . . . . . . . . . . . . . . . 91 


\section{List of Tables}

3.1 Summary of STK Parameters Applied in Analysis . . . . . . . . . . . . . 17

3.2 Best Case Link Budget . . . . . . . . . . . . . . . . . . . . . . 33

3.3 Worst Case Link Budget . . . . . . . . . . . . . . . . . . . . 35

3.4 Summary of Doppler Frequency Characteristics . . . . . . . . . . . . 36

4.1 Average Time to Measure BER for each Modulation Scheme . . . . . . . . . 43

4.2 Theoretical Bit Error Rate Equations . . . . . . . . . . . . . . . 47

4.3 Minimum $E_{s} / N_{0}$ Necessary to Achieve Selected BER Thresholds . . . . . . . 47

$4.4 E_{s} / N_{0}$ Upper and Lower Boundaries for a BER of $10^{-5} \ldots \ldots$

4.5 EVM-Estimated Bit Error Rate Equations . . . . . . . . . . . . . 56

4.6 EVM Thresholds for BER of $10^{-5} \ldots \ldots \ldots \ldots \ldots \ldots$

4.7 Error Vector Magnitude Bounds for Receiver Switching Scenarios . . . . . 59

A.1 Summary of Propagation Analysis Characteristics and Calculations . . . . 88 


\section{Chapter 1}

\section{Introduction}

\subsection{Motivation}

Traditionally, NASA communication links have been designed to operate during the worst-case, anticipated propagation conditions. These communication links are generally configured with a fixed data rate, a static modulation and/or coding scheme, and high link margins to communicate during the worst-case scenario. This worst-case planning approach ensures that communication links are maintained during even the worst expected Earth-to-Space propagation effects. These propagation effects include path loss, atmospheric loss, attenuation due to rain, and ionospheric loss, among others [1]. Another less-commonly investigated effect that can affect these communication links is antenna obstruction by either the satellite's

body or its solar panels [2]. Generally, a maximum bit error rate (BER) is identified in a communications system, and the link is designed to support that BER threshold. These propagation effects degrade the received signal power and are detrimental to a communications 
link if the BER increases above the acceptable limit for the transmitting waveform as the error correction codes can no longer resolve bit errors.

In the future, NASA envisions a more dynamic, flexible, and efficient communications network which optimizes mission data returns while minimizing the burden and cost of spacecraft resources and maintaining high fidelity (low BER) in the system. The fixed modulation, worst-case design approach often results in an underutilized system and limits flexibility during a variety of receiving scenarios, while ensuring the BER to be within the acceptable threshold. As a step towards this long-term goal, this work develops a link adaptation (LA) technique for the Earth-to-Space communications link between the NASA Space Communications and Navigation (SCaN) Testbed and the Glenn Research Center (GRC) ground station. During a lab-sponsored trip to GRC, the solar panels on the International Space Station (ISS) rotated through the antenna's field of view, causing significant degradation on the link. This observation inspired the application of a LA algorithm: allowing the receiver to select the modulation scheme that can maintain the desired BER and adapt as the signal energy fluctuates.

LA is a broad term to encompass many types of adaptation on a communications link. For instance, common types of LA that have been discussed and implemented are adaptive coding and modulation (ACM) as well as adaptive power allocation (APA) [3-5]. The algorithm suggested in this thesis monitors and switches modulation schemes depending on the signal strength on the communication link as it fluctuates throughout ISS passes. LA is used in a variety of terrestrial applications such as WiFi, land mobile communications, and 
cellular systems [3-8]. LA has also been used in satellite communications applications [9-12], evaluating the performance of an adaptive modulation system in the unique space-based propagation environment. However, these applications either present LA in a broadcasting system with no direct feedback to the ground station or do not consider shadowing effects. Some LA satellite applications consider weather impairments, such as rain-fading in the higher frequencies of Ka- and Ku-band [13] or log-normal shadow fading [14]. This work applies the ACM method of LA to address variations in the channel condition, most especially solar panel obstruction on the receiving antenna which can cause an interruption of data transmission.

\subsection{Contributions}

This work considers the SCaN Testbed system for propagation analysis and development of the LA algorithm. Chapter 2 introduces the communications link structure and configuration of the system to be analyzed. An introduction to the NASA SCaN Testbed and the Glenn Research Center (GRC) ground station are both provided as well. The proposed operation of the link adaptation logic is also introduced.

Chapter 3 defines the tools and propagation channel characterization necessary to develop a link adaptation algorithm. A summary of the software tools used, and the method in which they are applied, begins the chapter. An analysis of the passes visible to the ground station is presented, along with the associated path loss values. Then, a model of the point-to-point 
propagation path for the SCaN Testbed is presented. Common effects such as path loss and atmospheric loss are considered, as well as the less-commonly addressed power loss as a result of solar panel shadowing. Modeling of the solar panel shadowing is discussed, ultimately relating periods of obstruction to a shadowing loss parameter. Many additional system parameters used in the full characterization of the communications link are introduced with their calculations and assumptions. Finally, a full link budget analysis is performed for both the best-case and worst-case situations. The full link budget analysis summarizes the propagation model and includes the shadowing parameter, providing a characterization of the signal strength for application in the LA algorithm.

Chapter 4 introduces prior applications of link adaptation and discusses the broad nature of this area, and justifies the application of LA in this scenario. The LA algorithm is structured to operate the uplink path at a modulation scheme to maximize the data throughput, given a desired BER. An investigation into the decision metric used for this work's algorithm is presented. The focus is primarily on bit error rate (BER), signal-to-noise ratio (SNR), and error vector magnitude (EVM). Ultimately, EVM is the figure-of-merit used in the algorithm development. An analysis of the EVM decision thresholds is discussed and performed. The algorithm is then modeled to demonstrate the performance of EVM decisions versus true BER decisions.

Chapter 5 outlines the performance of the full system simulation using data generated from the analysis shown in Chapter 3 and the algorithm described in Chapter 4. The algorithm performance during periods of both solar-panel shadowing and shadow-free passes 
is presented. Additionally, a comparison to a fixed-modulation link and the link adaptation algorithm is presented, showing that the throughput of a fixed-modulation link can be improved with LA. Simulation analysis shows that the developed algorithm balances the trade-off between bit error rate and data rate as a function of the modulation scheme.

Chapter 6 summarizes the current state of the on-orbit receiver and transmitter design. Additionally, any further analysis or extension of the algorithm designs are outlined. Ultimately, given that the NASA SCaN Testbed on the ISS provides an ideal test environment for new satellite communication waveforms for NASA, the results of this work will be validated by an on-flight experiment on the Testbed as future work.

\subsubsection{Publications}

Kilcoyne, D. K.; Headley, W. C.; Leffke, Z. J.; Rowe, S. A.; Mortensen D. J.; Reinhart, R.C.; McGwier, R. W., "Link Adaptation for Mitigating Earth-to-Space Propagation Effects on the NASA SCaN Testbed," IEEE Aerospace Conference, 2016.

Beggs, D. K.; McGwier, R. W., "Mitigating Earth-to-Space Propagation Effects through Adaptive Waveform Switching," 2015 AMSAT Space Symposium and Annual Meeting. October 2015. Dayton, OH. 


\section{Chapter 2}

\section{System Configuration}

This chapter outlines the system configuration for the research presented in this thesis. An introduction to the SCaN Testbed, the platform used in the analysis, and in-flight experiment is presented. The communications link structure is also outlined, as a solid understanding of the experimental set-up is necessary for the propagation analysis and algorithm development, discussed in future sections.

\subsection{SCaN Testbed Overview}

The Space Communications and Navigation (SCaN) Program Office manages all NASA space communication efforts, such as the Deep Space Network (DSN), Near Earth Network (NEN), and the SCaN Testbed. The NASA SCaN Testbed was developed and proposed as a research initiative to explore the application of software-defined radios in space communications. The Testbed allows NASA, industry, and NASA academic partners to develop and test 


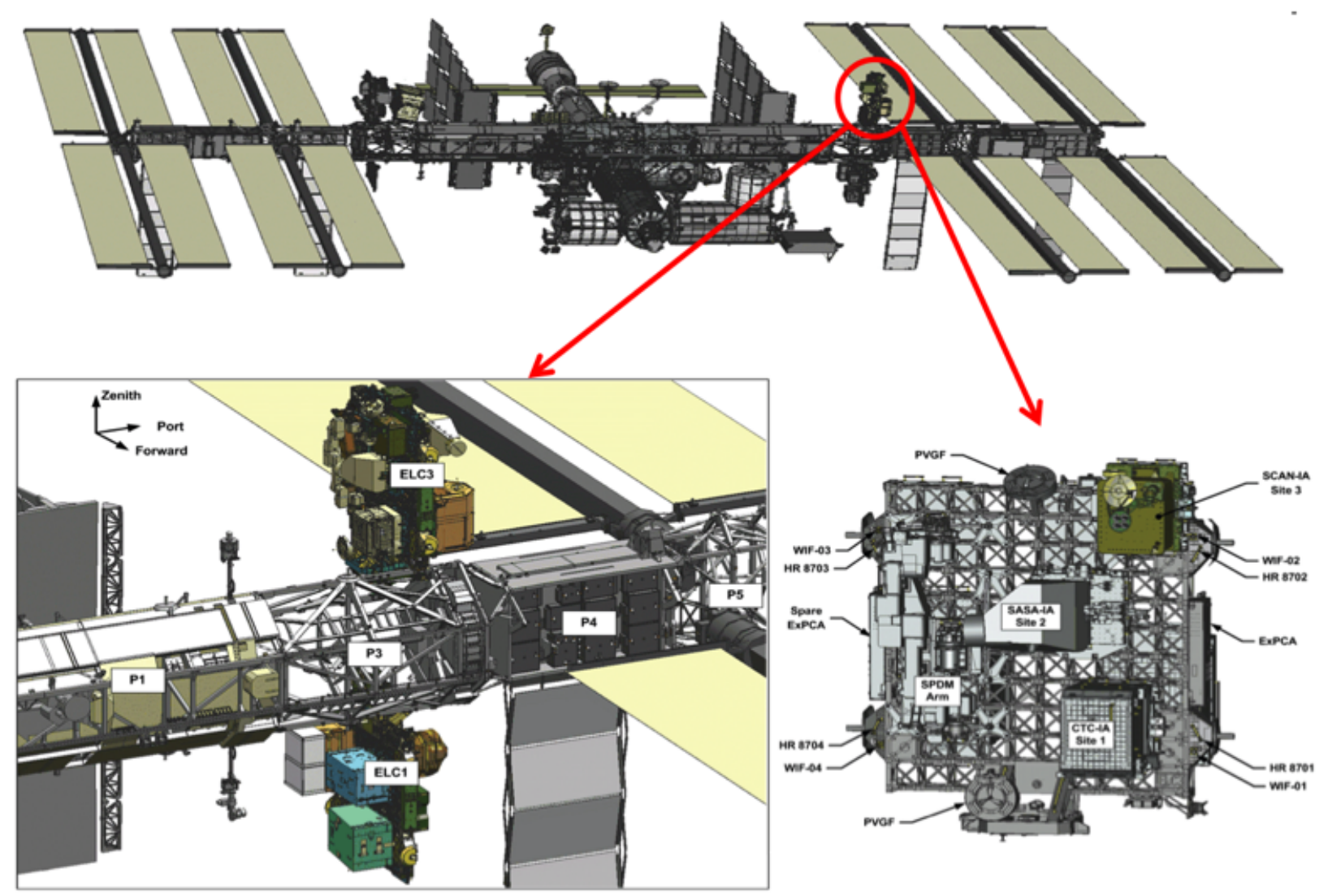

Figure 2.1: Position of NASA SCAN Testbed on-board the ISS ELC.

communications waveforms and networking protocols on a reconfigurable platform. The SDR platform provides a cost-effective method to validate designs and technologies. The SCaN Testbed platform launched and installed on the International Space Station (ISS) Equipment Logistics Carrier-3 (ELC3), as seen in Fig. 2.1 [15].

The Testbed has three SDRs installed on-board, operating at L, S, and Ka-band which correspond to frequency ranges 1-2 GHz, 2-4 GHz, and 27-40 GHz, respectively [16]. Not all these frequency ranges have direct-to-ground capability as many of them communicate directly with NASA's Tracking and Data Relay Satellite System (TDRSS). Two antennas have tracking capability for the TDRSS satellites, with three additional fixed antennas. One 
fixed antenna receives GPS signals, with the other two operating at S-band. The ram-facing ${ }^{1}$ S-band antenna has direct view to the ground station at Glenn Research Center (GRC). This antenna and its associated radio, the Jet Propulsion Laboratory (JPL) Software Defined Radio (SDR), will be the equipment used in this work.

\subsubsection{On-board JPL SDR}

The JPL SDR, launched to the ISS in 2012, is a S-band reconfigurable transceiver used in the propagation modeling and simulation [17]. This software-defined radio is equipped with both a SPARC general purpose processor (GPP) and two Xilinx Virtex II field programmable gate arrays (FPGAs) to perform waveform applications developed by experimenters. The GPP and Xilinx FPGAs are the user-programmable components and will host the receiver and algorithm designed in this work. As the Virtex II FPGAs are limited in the multiplies available, the receiver and algorithm design must factor in computational constraints.

\subsection{Glenn Research Center Ground Station}

The Glenn Research Center, located in Cleveland, OH, brought a ground station on-line in early 2015 to support the research efforts of the SCaN Testbed. As the Testbed has both relay satellite and direct-to-ground capabilities, the facility at Glenn serves as an additional direct-to-ground research station.

\footnotetext{
${ }^{1}$ ram-facing: pointing in the direction of satellite velocity
} 


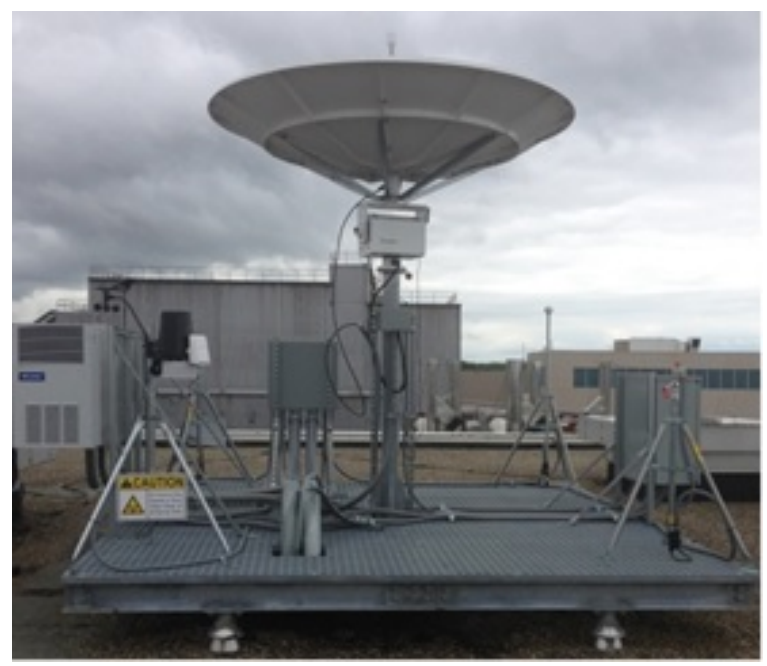

Figure 2.2: S-band ground station antenna at GRC, $2.4 \mathrm{~m}$ dish.

The S-band antenna at the ground station, seen in Fig. 2.2, is a $2.4 \mathrm{~m}$ parabolic antenna. The antenna is networked to the SCaN Testbed operations center, with full orbital prediction used for antenna tracking commands. Additionally, GRC has software tools that estimate system parameters for the communications link including: bit error rate (BER) performance, ISS link obscuration, and power levels. The data returned from this tool is intended to be employed as a performance metric for this work after on-orbit experimentation. The GRC ground station also holds a prototype of the flight SDR for verification and validation testing before on-orbit experimentation.

\subsection{Communications Link Configuration}

For radio signals, propagating to space requires deliberate and meticulous planning, because of the propagation effects that must be considered. In this work, the link between the GRC ground station and the SCaN Testbed flight SDR on-board the ISS is analyzed as the scenario 


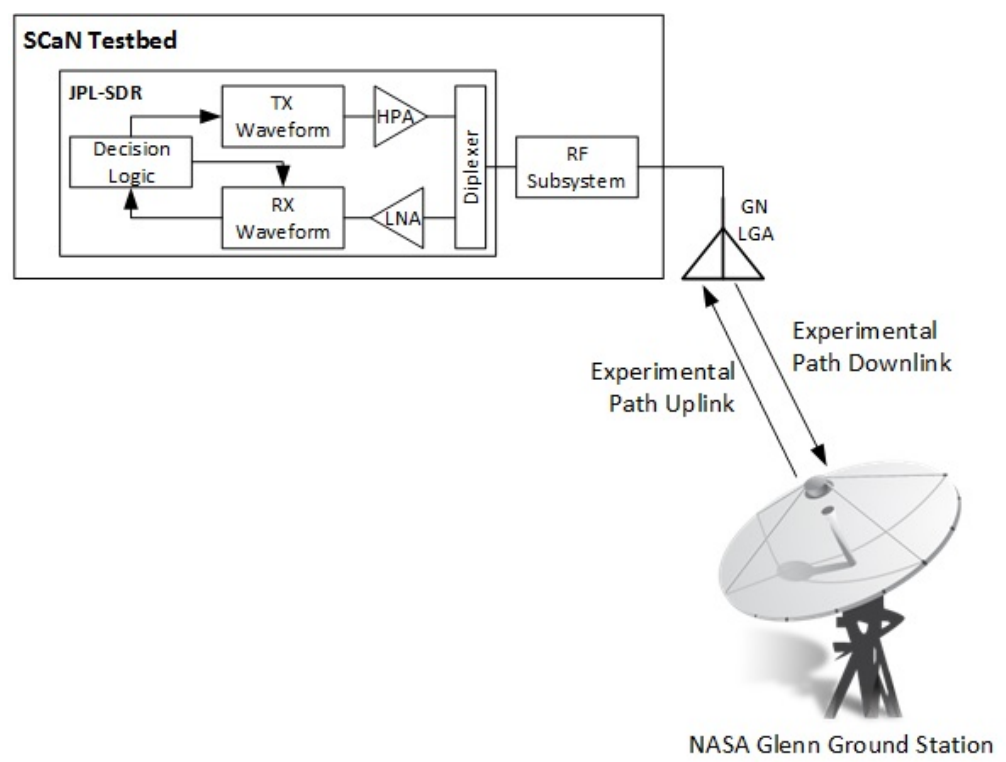

Figure 2.3: System configuration of communications link.

communications link. Fig. 2.3 depicts the configuration of the link assessed in this work. The Experimental Path Uplink originates at the GRC ground station and communicates to the flight SDR antenna. Conversely, the Experimental Path Downlink originates at the flight SDR and is received at the GRC ground station. Within the flight SDR, both receiving and transmitting functionalities are employed with the algorithm decision logic analyzing the status of the link. The sections below expand upon the different stages of the communications link.

\subsubsection{Experimental Path Uplink}

The Experimental Path Uplink originates at the GRC ground station, transmitting data to the flight SDR. The link layer framing protocol CCSDS is tentatively selected because 
it is an accepted standard in space communications. The link will be adaptive; switching modulation schemes depending on the channel conditions. For the uplink, Binary Phase Shift Keying (BPSK), Quadrature PSK (QPSK), 8-PSK, 16-Quadrature Amplitude Modulation (16-QAM) and 64-QAM are the chosen modulation schemes from which the algorithm can select. All modulation schemes are Gray-coded with a fixed symbol rate of $502 \mathrm{kSps}$. These five modulation schemes are the five lowest order modulation schemes and can operate in lower link margins.

While some link adaptation techniques alter both modulation and coding as discussed in Chapter 1, the data will be uncoded in this analysis. Remaining uncoded simplifies the FPGA receiver design and also addresses only one adaptation implementation on the hardware at a time. Future work would incorporate both adaptive coding and modulation.

Additionally, as is the case with many moving receivers, Doppler effect is considered on the uplink. The transmitter at GRC will account for Doppler shift and adapt the uplink frequency as the ISS moves over the ground station.

\subsubsection{Decision Logic}

On-board the SCaN Testbed, the receiver will receive and demodulate the transmitted data. During the demodulation, the FPGA will calculate an error vector that will be used in the channel condition analysis. The on-board GPP will then estimate the bit error rate (BER) through the application of the calculated error vector magnitude. If the estimated BER of the received data stream is above a predetermined threshold, the decision logic within 
the GPP will determine what modulation waveform can deliver the desired fidelity while maximizing data rate for the current channel conditions. Similarly, if the estimated BER is well-below the desired threshold, the decision logic will determine which modulation scheme maximizes data throughput while remaining below the BER threshold.

Information about the currently necessary modulation scheme, along with additional statistics such as the estimated error vector magnitude (EVM), will be modulated and transmitted to the ground station on the Experimental Path Downlink.

\subsubsection{Experimental Path Downlink}

The Experimental Path Downlink will transmit a robust Binary Phase Shift Keying (BPSK) signal so as to maintain high fidelity. As this link acts as the feedback loop, it is necessary to maintain a very low BER regardless of the channel conditions. Thus, BPSK was selected as the modulation scheme as it is the most robust of the digital modulation schemes in low SNR conditions [18]. Additionally, a high data throughput is not a concern for the downlink.

This link transmits the status updates from the flight SDR, specifically the current mode to be operating on. For example, should the GPP determine that the GRC transmitter should switch to QPSK from 64QAM, the BPSK downlink packet stream will adjust to indicate QPSK as the new modulation scheme. The GRC ground station will have a BPSK receiver monitoring the status updates from the JPL SDR on the downlink and will adjust the uplink modulation schemes accordingly. 
As with most feedback loops, there is a delay between when the on-board logic determines that a switch should occur and when the GRC ground station transmitter is able to start transmitting the desired modulation scheme ${ }^{2}$. When the receiver is switching up a modulation scheme, the GRC transmitter will begin transmitting data modulated at the higher modulation scheme directly. However, when the receiver switches down modulation schemes, it is assumed that the bit error rate performance has degraded, causing errors. To address any data that might have been demodulated incorrectly and taking into account the round-trip delay, the transmitter will re-transmit the data that was sent when the channel conditions changed.

\footnotetext{
${ }^{2}$ The longest round-trip delay is $15.6 \mathrm{~ms}$, when the ISS is at a range of $2328.67 \mathrm{~km}$.
} 


\section{Chapter 3}

\section{Propagation Analysis}

Satellite communications provide a unique propagation environment to navigate when transmitting signals to and from an orbiting vehicle. Path loss, atmospheric losses, rain fade, ionospheric effects (such as scintillation), and other effects can greatly affect the received signal strength. The communications link discussed in Chapter 2 experiences many of these effects. Additionally, the solar panel obstruction of the antenna periodically introduced on this link must also be modeled and analyzed. All these considerations lead to a full link budget analysis, which is then leveraged to develop the link adaptation (LA) algorithm. This chapter discusses the tools applied in this analysis and presents calculations of an estimated link budget ${ }^{1}$. The estimated link budget provides the characterization necessary to understand the conditions of the communications link.

\footnotetext{
${ }^{1}$ Due to the export-controlled nature of this work, some parameters within the analysis are estimated or approximated by similar prior work to present the function of the proposed LA system without compromising proprietary information.
} 


\subsection{Software}

This section outlines the tools employed to model the propagation environment. For the purposes of the environment characterization, the scenario modeled was a one month period. This duration allowed for 207 individual views to the GRC ground station. The time stepped in 1 second increments.

\subsubsection{Systems Toolkit}

This work leverages the Analytical Graphics, Inc. (AGI) Systems Toolkit (STK) software for acquiring many of the parameters necessary for characterization of the communication link environment. STK is a tool used by many engineers, mission analysts, and software developers to model systems such as aircraft, missiles, and satellites. These capabilities are supported by databases of flight plans and orbit paths. The International Space Station (ISS) orbit is easily loaded after searching the STK satellite database by common name. STK uses Two Line Elements (TLE) and the SGP4 propagator to model the orbit, see Appendix for definition of TLE and SGP4. On an instantiated satellite, antennas and sensors can be defined. An antenna and sensor are both installed at the JPL SDR location on ELC-3, which allows for accurate simulations of both the uplink and downlink.

The sensor is used in the calculation of accesses. Accesses in STK are defined as times during a satellite orbit when the satellite is visible to a target. In this scenario, the target is the GRC ground station facility. The facility is defined and established at the approximate 


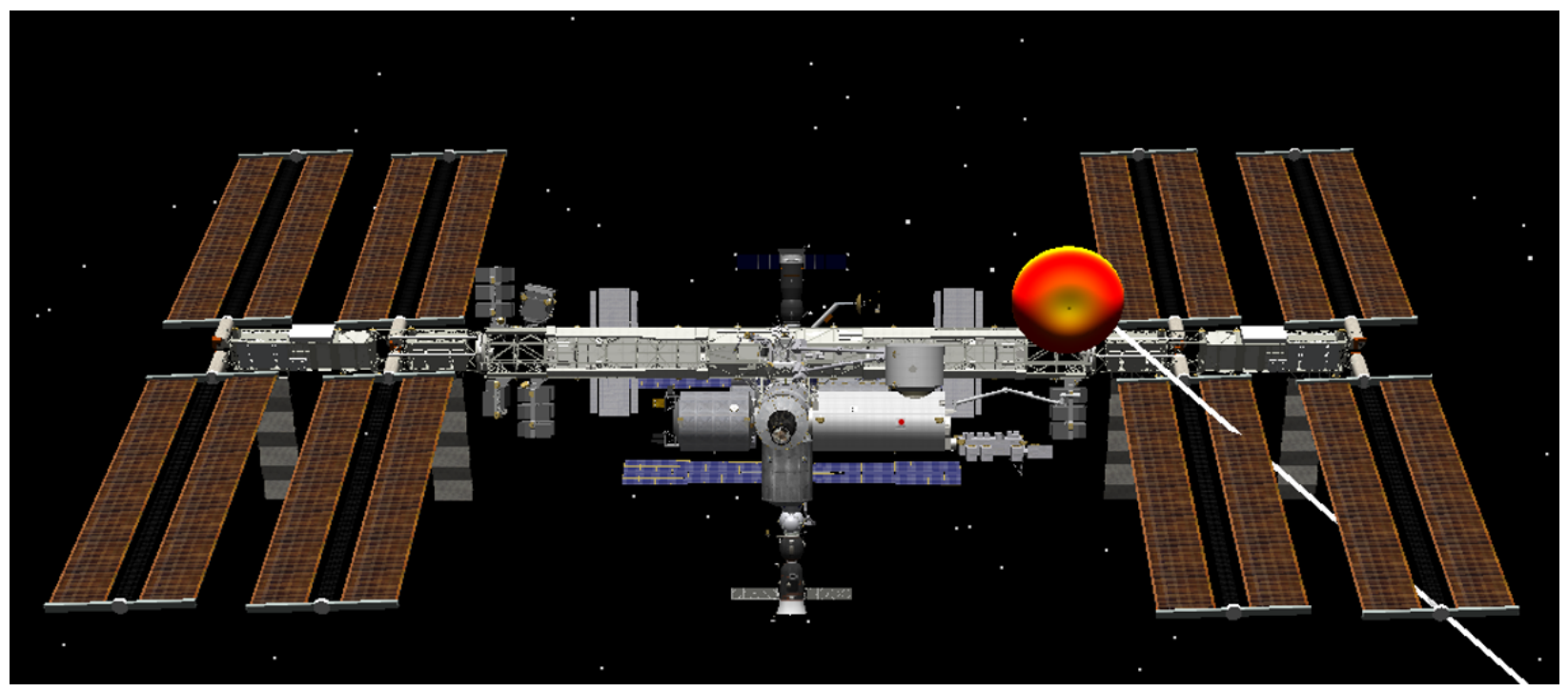

Figure 3.1: Flight antenna obstructed by solar panels.

latitude and longitude of GRC in Cleveland, $\mathrm{OH}$.

A generic STK-provided antenna pattern modeling a GPS Fixed Reception Pattern Antenna (FRPA) is used to approximate the changing receiver antenna gain as the ISS moves through orbit. The FRPA is the closest approximation within STK for the restricted JPL SDR antenna pattern. The FRPA antenna is a low-gain antenna with a hemispherical pattern; similar to the actual SDR antenna. This antenna pattern gives a more realistic model of the anticipated receiver gains which vary with both azimuth and elevation, as seen in the receiver gain matrix in Section 3.4.2.

Additionally, the antenna object is utilized to determine the times during which the solar panel rotated in front of the antenna. As an example, Fig. 3.1 is an STK illustration of the solar panel blocking the line-of-sight of the SCaN Testbed antenna to the ground station. 
Table 3.1: Summary of STK Parameters Applied in Analysis

\begin{tabular}{|c|c|}
\hline \multicolumn{2}{|r|}{ Access Parameters } \\
\hline Access Number & This parameter tracks the current access number of the simulated timeframe. \\
\hline Epoch Time (sec) & This value provides the epoch time at each data point with the access. \\
\hline Range $(\mathrm{km})$ & The distance from the SCaN Testbed onboard the ISS to the ground station. \\
\hline Range Rate (km/sec) & The velocity of the ISS along line-of-sight vector to GRC. \\
\hline Azimuth (deg) & The direction, in degrees, offset from the ISS ram vector to the GRC ground station [19] \\
\hline Elevation (deg) & Angle, in degrees, down from ISS ram vector [19]. \\
\hline Receiver Gain Matrix (dBi) & Gain matrix of flight SDR antenna as a function of azimuth and elevation. \\
\hline \multicolumn{2}{|r|}{ Solar Panel Obstruction } \\
\hline Epoch Time (sec) & This value provides the time at which each obstruction point is measured. \\
\hline Percent Obscured Area & This value is returned from the obscuration tool discussed in Section 3.3.1. \\
\hline
\end{tabular}

STK provided many of the simulated values used in the full propagation analysis. Table 3.1 displays a summary of these values.

\subsubsection{MATLAB}

STK has a MATLAB interface capability, which enables users to instantiate satellites, ground stations, receivers, antennas, etc. and then perform analysis on these objects in MATLAB. A MATLAB-STK interface is used to set-up the ISS, GRC ground station, SCaN Testbed receiver, and antenna and calculate the accesses for the scenario.

The full link budget analysis also uses MATLAB for characterization of shadowing obscuration, individual access parameters, and ultimately the algorithm modeling. The analysis incorporates the values generated through the STK simulation, such as path loss and the receiver antenna gain. Additionally, values such as the effective isotropically radiated power (EIRP), receiver losses, atmospheric losses, etc. are all incorporated into the analysis using MATLAB. 


\subsection{Path Loss}

Path loss is the largest loss experienced during satellite communications. Path loss is critical to include in a full link budget as it causes a variable received signal power across each satellite pass as a function of range. With each pass over the ground station having a different path loss curve, many values of received signal strength are experienced by the flight SDR on board the ISS. Section 3.2.2 describes how path loss was calculated. Before calculating path loss however, a discussion on the variation of passes is presented in Section 3.2.1.

\subsubsection{Path Overview}

First, an understanding of when an access occurs is imperative towards the understanding of the passes analyzed in this work. Passes are assumed to be accesses only as the ISS is visible to the GRC ground station. For the purposes of this analysis, an access is defined as the period of time during the ISS orbit that corresponds to a positive elevation angle, measured relative to GRC. This positive elevation angle indicates when the ISS has crested the local horizon. In this definition, accesses are defined purely on the geometry of the orbit; not the acquisition-of-signal (AoS) and loss-of-signal (LoS). Restrictions on the elevation angle, either as dictated by the National Telecommunications and Information Administration (NTIA) or by local geographic features (mountains, buildings) that obscure the line-of-sight, are not considered [19].

The month-long simulation in STK includes 207 accesses. The shortest pass is 0.2 


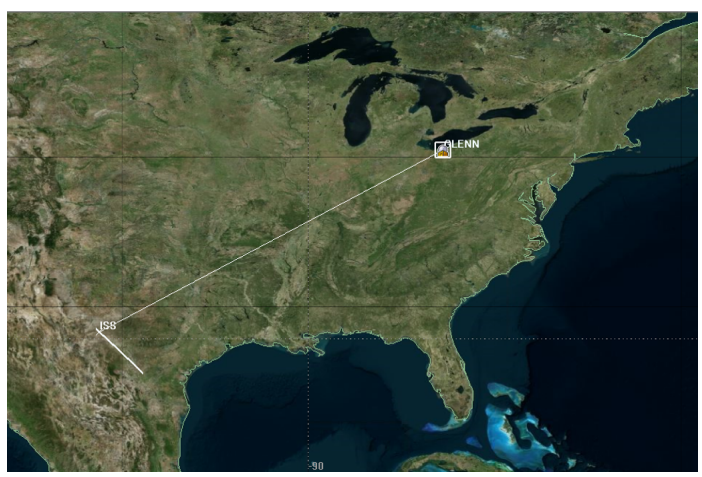

(a) Ground track of shortest access.

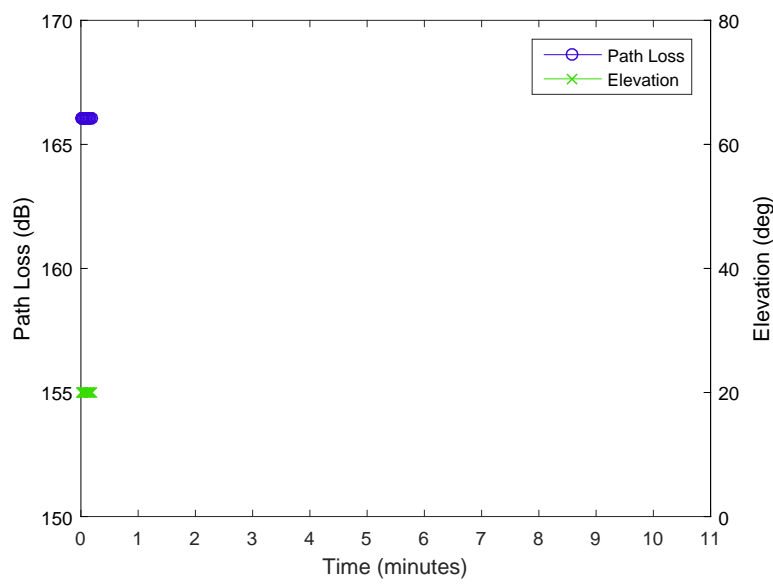

(c) Path loss and elevation of shortest access.

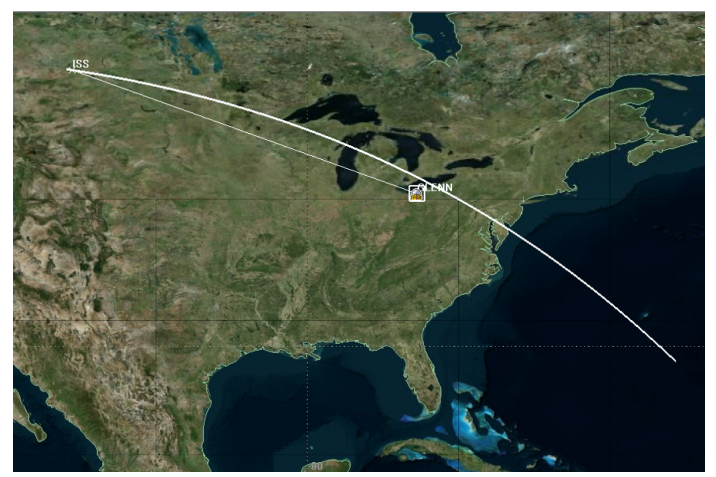

(b) Ground track of longest access.

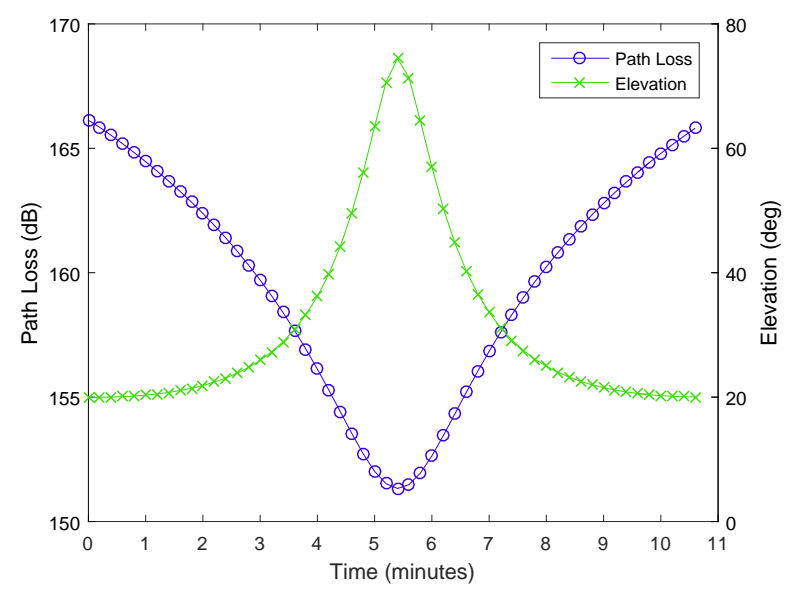

(d) Path loss and elevation of longest access.

Figure 3.2: Simulated ground track, path loss, \& elevation for shortest \& longest accesses.

minutes, the longest is 10.78 minutes. Figure 3.2 displays the ground track for the ISS during both the shortest and longest passes.Observe that the position of the ISS during its orbit and the elevation angle ${ }^{2}$ (plotted on the right axis in Figs. 3.2c and 3.2d) affect the length of the available communication time with the GRC ground station. Out of the 207 considered accesses, $95.6 \%$ of the passes are longer than 4 minutes in duration.

\footnotetext{
${ }^{2}$ The elevation angle is measured from GRC to the ISS
} 


\subsubsection{Path Loss Calculation}

Range data from STK analysis was used to estimate the range value used in calculating path loss. The wavelength of the signal is also used in the determination of path loss. The flight SDR is operating at $2.07 \mathrm{GHz}$ (within S-band) on the uplink so the wavelength, $\lambda$, is found

$$
\lambda=\frac{c}{f},
$$

where $c$ is the speed of light in free space $\left(3 \times 10^{8} \mathrm{~m} / \mathrm{s}\right)$ and $f$ is the transmitting frequency. The resulting wavelength is $0.14 \mathrm{~m}$.

Across each pass, the range of the flight SDR to the ground station is constantly changing as the ISS continues through orbit, as seen in Fig. 3.3. Therefore, the path loss is not a constant value and is calculated at each time increment during the simulated pass. For characterization of the full scope of path loss, the maximum and minimum path loss values are calculated.

The path loss values found in Fig. 3.3 are calculated through

$$
L_{P}=10 \log _{10}\left(\frac{4 d \pi}{\lambda}\right)^{n}
$$

where $n$ is the free-space path loss exponent equal to 2 and $d$ is the range from the ISS to the ground station. The calculated maximum path loss value is $166.1 \mathrm{~dB}$ and the minimum is $151 \mathrm{~dB}$. The path loss characteristics are summarized in the Appendix in Table A.1.

Figure 3.3 displays how the path loss changes over time for a representative 60 out of 


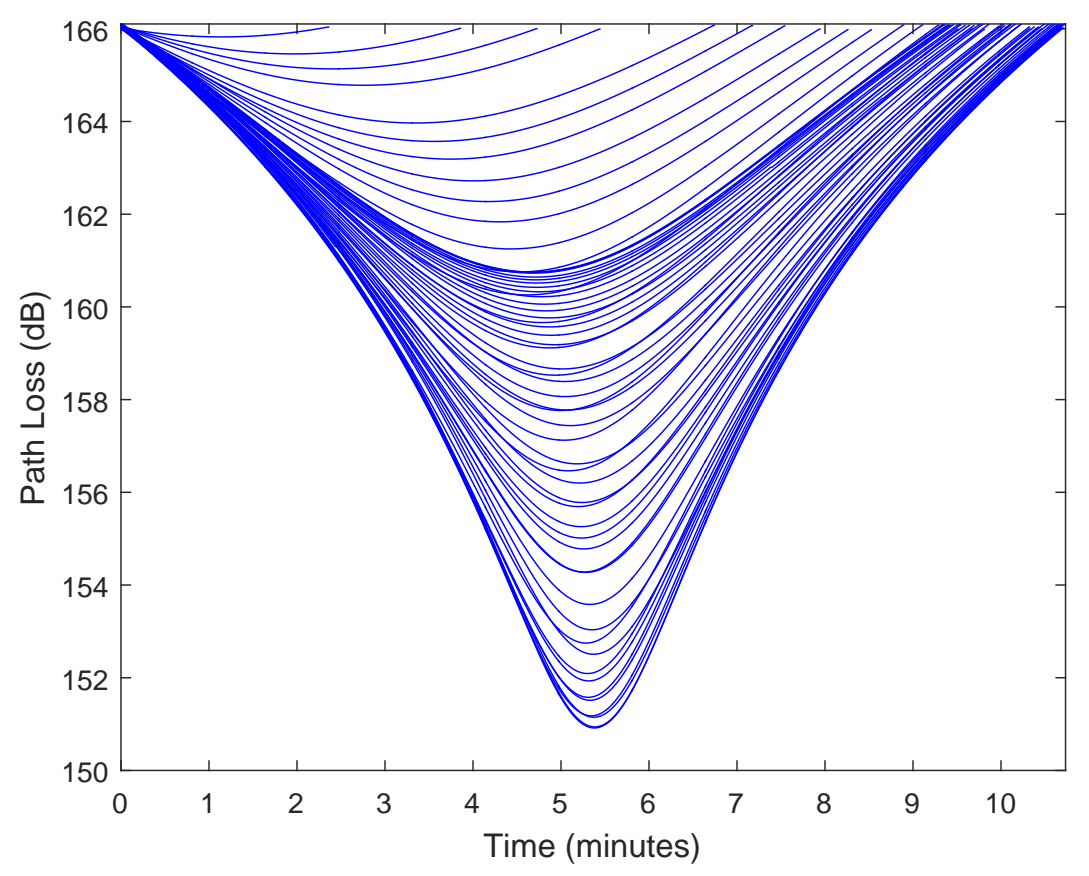

Figure 3.3: Time-varying path loss for 60 accesses.

the 207 accesses over the one month period. This path loss graph depicts the entirety of the pass that the ISS is visible to the ground station; however the flight SDR will not have the same visibility times due to the fixed directional antenna. The flight SDR antenna used in this work is a ram-facing antenna-it faces in the direction of the satellite motion. Once the ISS has passed over the GRC, traveling west to east, the antenna will not be able to track or look-back towards the ground station; the receiver antenna gain matrix discussed in Section 3.4.2 accounts for the roll-off in antenna gain as the elevation angle changes. 


\subsection{Shadowing}

Providing electricity to the ISS is critical for maintaining full operation. To maximize power generation, the solar panels of the ISS are mounted on gimbals so as to track the sun, such that the incident angle of the sun is orthogonal to the panels [20]. The constant motion of the solar panels can cause conflicts with stationary communication antennas. The flight SDR antenna is positioned on the ISS in a way that causes it to be blocked by the solar panels, as was shown in Fig. 3.1. Currently, during periods of obstruction, little transmission occurs, and when it does, engineers only operate the most robust of waveforms. This work addresses the goal to maintain data transmission during periods of solar panel shadowing while, at the same time, maximizing the data throughput. To do so, a model of the ISS solar panel shadowing must first be developed.

Prior work has shown that an antenna blocked by solar panels experiences a degradation loss of $6 \mathrm{~dB}$ [21]. While this prior work investigated an antenna with a different pattern, a similar approach can be applied to this work. When the solar panel is blocking the flight SDR antenna, a fixed degradation as a result of shadowing can be included in the link budget analysis for the LA algorithm development. In order to determine when the degradation is in effect, a method for identifying when the solar panel shadowing occurs must first be developed. 


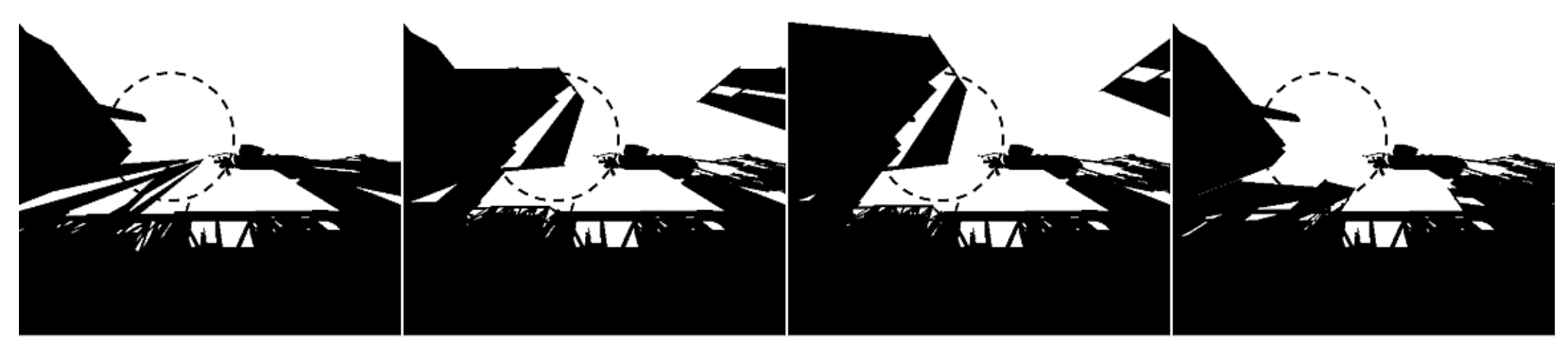

Figure 3.4: STK obscuration tool showing stages of a solar panel rotation.

\subsubsection{Obscuration Tool}

STK incorporates the sun-tracking of solar panels in the simulation, so the rotations are visible as the simulation progresses. In order to identify when the solar panels obstruct the S-band antenna, the STK Obscuration Tool is employed. This tool provides the view down the antenna boresight and calculates the percentage of the boresight view that is blocked. When identifying an area as obscured, the tool does not differentiate between the ISS body or solar panels. A recent work applied the STK obscuration tool in an assessment of ENVISAT antenna obstruction [2] by leveraging the tool's percentage of field-of-view that is obstructed. The shadowing model for this work follows a similar approach, although adjusting for the constant ISS body that should not be classified as shadowing. Fig. 3.4 depicts the tool during a few stages of solar panel rotation, during which the solar panel rotates through the field of view at discrete time steps. The obscuration tool takes into account the pointing angle to the ground station, but solely estimates the percentage of area obstructed. As a result, a threshold for the percentage-obstructed-area was selected to classify the antenna as shadowed and thus incorporate the shadowing loss into the link budget. 


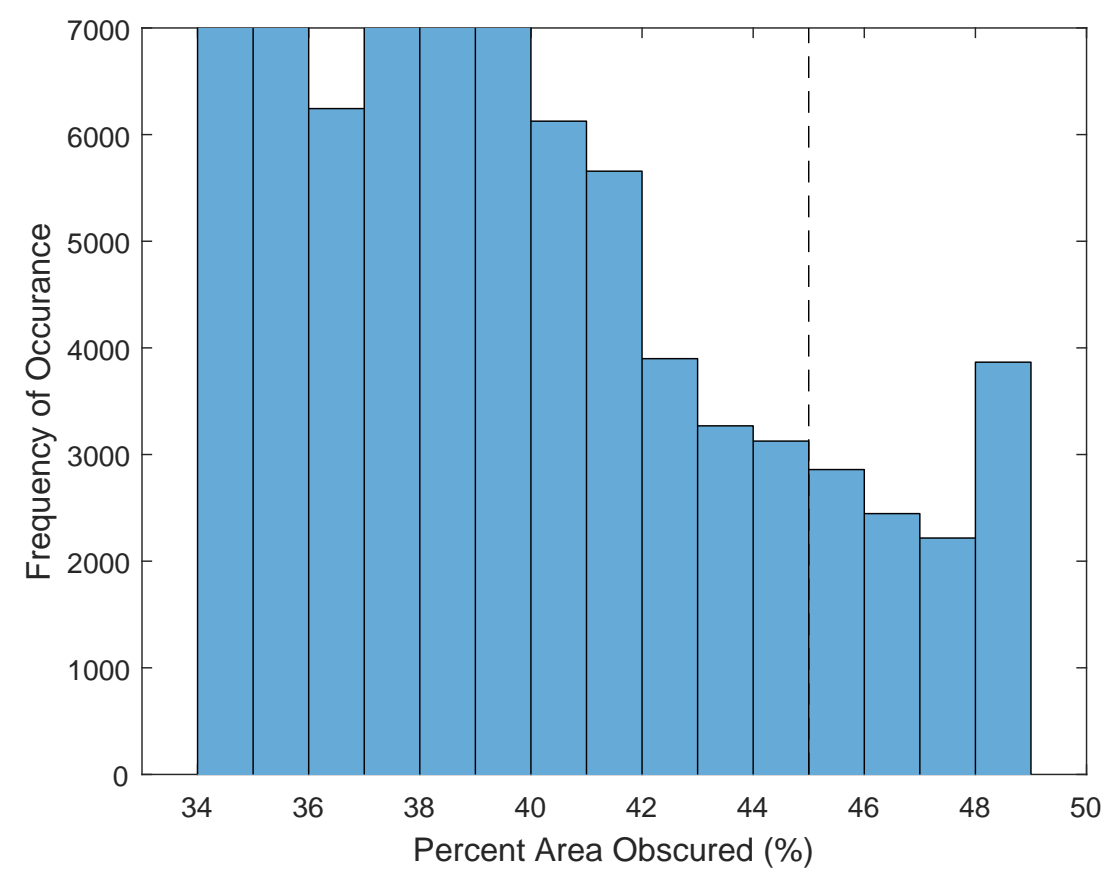

Figure 3.5: Histogram of percentage area-obscured from STK obscuration tool.

Figure 3.5 shows a histogram of the percent-obscured values across all 207 accesses analyzed for this scenario. Because the ISS body is a constant in the obscuration tool, the minimum percent area obscured is $33.6 \%$. As seen in Fig. 3.5, most of the time, the percent-area-obscured is very close to the baseline level. The threshold used to classify the current access as shadowed is $45 \%$ (shown as the dashed line in Fig. 3.5), as that value identifies approximately $20 \%$ of all accesses to have shadowing, an approximate percentage observed during operation at GRC. The maximum obscuration is $49.1 \%$. The threshold allows for a fluctuation of $4 \%$ as the solar panel moves through the antenna boresight.

Within the link budget analysis, a shadowing loss will be incorporated should the pass be identified as "shadowed", using the threshold discussed above. The shadowing loss is a 
function of the obstruction percentage, garnered from the STK obscuration tool. Because the solar panels are not static and they are constantly rotating, the shadowing loss should not be a static variable either, and instead a function of the percentage-area-obscured. The max shadowing loss assumed is $10 \mathrm{~dB}$; a conservative estimate [21] and in line with observations at the GRC ground station. The maximum obscuration is $49.1 \%$, so at that moment, the shadowing loss will be $10 \mathrm{~dB}$. To relate the percentage-area-obscured and shadowing loss

$$
L_{\text {shadowed }}=\frac{C_{\text {shadowed }} \times P_{\text {obscured }}}{100},
$$

where $C_{\text {shadowed }}$ must be $10 \mathrm{~dB}$ at the max value of $P_{\text {obscured }}$ given by the obscuration tool, as that correlates to maximum shadowing loss. The max percentage obscured is $49.1 \%$. Thus, $C_{\text {shadowed }}$ is $20.4 \mathrm{~dB}$.

$$
L_{\text {shadowed }}=\frac{20.4 \mathrm{~dB} \times P_{\text {obscured }}}{100}
$$

\subsubsection{Shadowing Loss}

Figure 3.6 displays all shadowing losses for the 207 accesses. 56 of the passes are identified as having some associated shadowing, all varying with time.

Out of the 56 shadowed passes, two-thirds experience shadowing for $50 \%$ or less of the total access duration. Only $8.9 \%$ of shadowed access are $75 \%$ or more of the total access duration. These statistics show that during a pass, the solar panels do not generally obstruct 


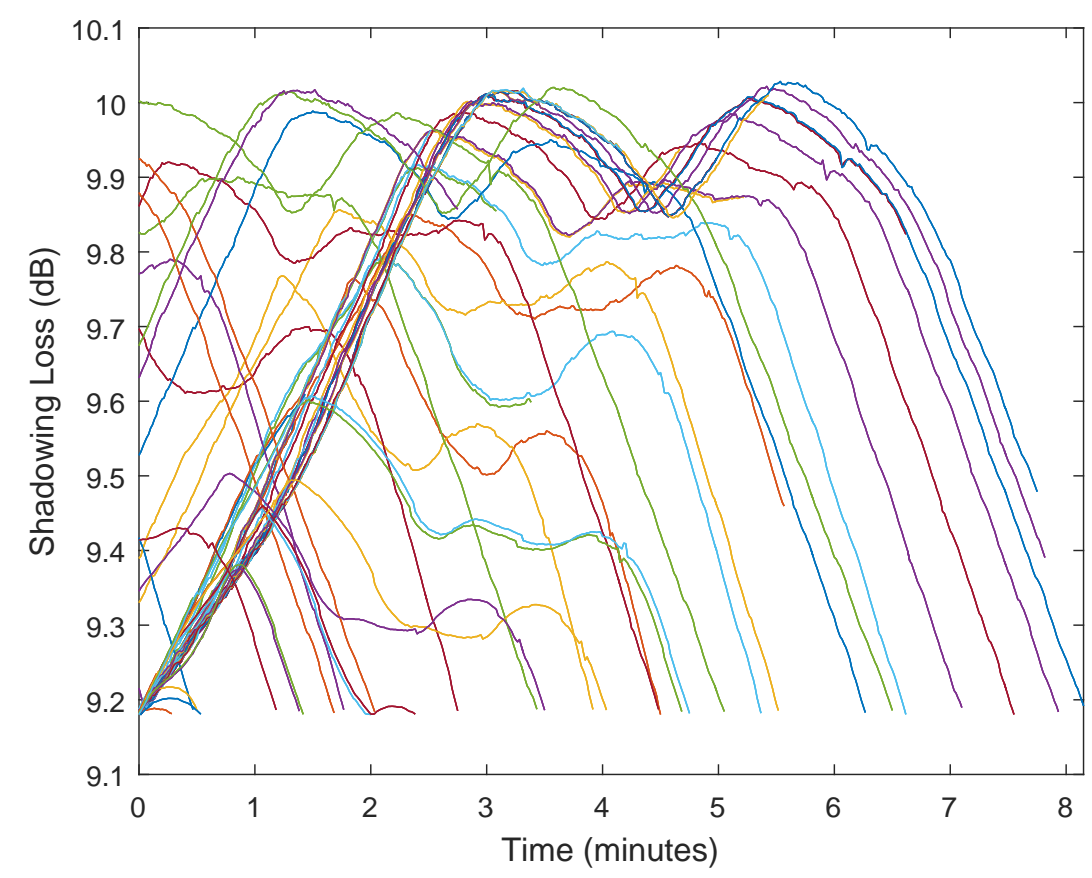

Figure 3.6: Shadowing loss curves for all obstructed accesses.

the antenna through the entire access, but rather rotating in and out of view during an access.

\subsection{System Parameters}

Aside from path loss and shadowing loss, several other parameters must be defined before performing a full link budget calculation. This section introduces the rest of these parameters and the calculations for the link budget. 


\subsubsection{Transmitter Antenna Gain}

The GRC ground station has a $2.4 \mathrm{~m}$ parabolic dish antenna used at the transmitter in the Experimental Path Uplink. The ground station transmitting antenna gain is given by

$$
G_{T X}=10 \log _{10}\left(\eta\left(\frac{d \pi}{\lambda}\right)^{2}\right)
$$

with $d$ defined as the diameter of the antenna and $\lambda$ as the wavelength, numeric values outlined in Table A.1. Given an approximated efficiency, $\eta$, of $70 \%$, a commonly accepted efficiency for a well-designed and optimized parabolic antenna [22], the gain of the transmitter antenna was found to be $32.5 \mathrm{dBi}$.

\subsubsection{Receiver Antenna Gain}

The receiver antenna gain, $G_{R X}$, is dependent on the azimuth and elevation of the satellite as it passes over the ground station due to a fixed, known pointing angle. Figure 3.7 displays the antenna pattern volume modeled in STK. The shading intensity corresponds to magnitude of the receiver antenna gain.. To relate this graphical representation to a format compatible with the link budget calculations, a receiver antenna gain matrix is generated through STK. In STK, a matrix of receiver antenna gain values is generated for azimuth values between -180 and 180 degrees and elevations between -90 and 90 degrees. Each cell within this matrix corresponds to an individual azimuth and elevation pair of the receiving antenna. In simulations of each access, the receiver gain is selected from the matrix using the azimuth 


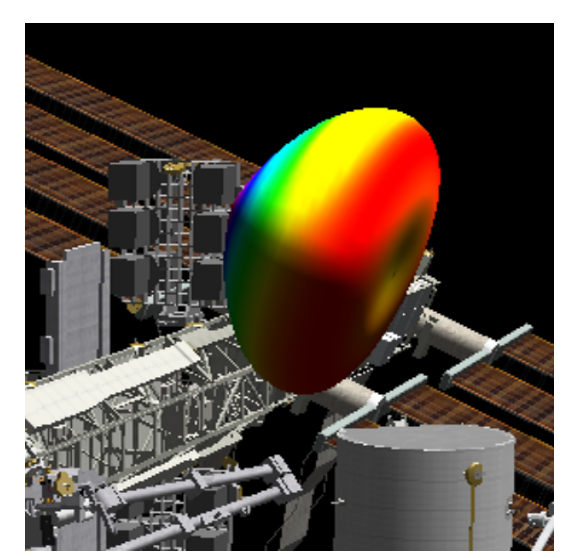

Figure 3.7: STK RX antenna for gain estimation.

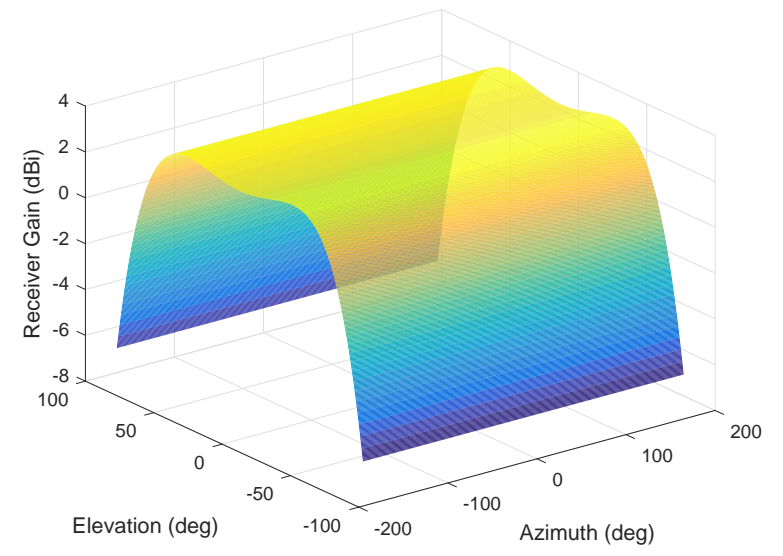

Figure 3.8: RX antenna gain matrix.

and elevation pair for each time step. Figure 3.8 demonstrates the graphical representation of the STK-generated matrix, as a function of both azimuth and elevation.

\subsubsection{Receiver Noise Temperature}

Receivers all have an inherent thermal noise component which introduces additional noise to the signal. Common receivers have a noise figure of $3 \mathrm{~dB}$ [23]. The noise figure of the flight SDR is thus conservatively estimated at $5 \mathrm{~dB}$, to overestimate the actual noise figure. Overestimating provides some allowance in the final model. To determine the receiver noise temperature,

$$
T_{R X}[\mathrm{~K}]=T_{\text {ref }}\left(10^{\frac{N F_{d B}}{10}}-1\right)
$$

where $T_{\text {ref }}$ is the nominally accepted $290 \mathrm{~K}[16]$ and giving a receiver noise temperature of $432.5 \mathrm{~K}$. 


\subsubsection{System Noise Temperature}

The receiver noise temperature in Section 3.4.3 is used in conjunction with the antenna noise temperature to calculate the total system temperature. The antenna temperature is estimated at $200 \mathrm{~K}$ [24] to calculate the system noise temperature for the noise power in the link budget. Using the system temperature values summarized in Table A.1, the receiver noise temperature is calculated through

$$
T_{\text {sys }}[\mathrm{K}]=T_{a n t}+T_{R X},
$$

giving a system temperature of $632.5 \mathrm{~K}$.

As the antenna changes looking angles through passes, it may look into the sun, causing the temperature to increase significantly beyond the $200 \mathrm{~K}$ estimation. This estimation is a general, average value-specific sun effects can be investigated for a future model.

\subsection{Link Budget}

The link-budget calculation is the core analysis tool for the characterization of the propagation channel. Link budgets are employed for radio links that rely on line-of-sight propagation through space [25]. In this model, each link budget of an access varies due to the changing values of range and receiver gain as a function of time. However, initially, the static variables associated with the full link budget calculation are outlined. 


\subsubsection{Received Power}

The Friis Transmission Equation, given by

$$
P_{R}[\mathrm{dBW}]=P_{T}+G_{T}+G_{R}-L_{P}
$$

relates the received power at the flight SDR to the transmitted power from GRC [25]. This equation is modified below to include additional losses that are taken into account for this model.

$$
P_{R}[\mathrm{dBW}]=P_{T X}+G_{T X}-L_{T X}+G_{R}-L_{R X}-L_{P}-L_{\text {atmo }}-L_{\text {iono }}
$$

First, the Effective Isotropic Radiated Power (EIRP) describes the power radiated uniformly in all directions from the transmitter; it is the combination of the transmitter power, transmitter gain, and any losses incurred at the transmitter [25].

The EIRP is found to be $40.5 \mathrm{dBW}$ using the parameters of the transmitting ground station defined in Table A.1 through

$$
\operatorname{EIRP}_{T X}[\mathrm{dBW}]=P_{T X}+G_{T X}-L_{T X} .
$$

Following the calculation of the $\operatorname{EIRP}_{T X}$, the received power is calculated through substituting $\operatorname{EIRP}_{T X}$ into (3.9),

$$
P_{R}[\mathrm{dBW}]=\mathrm{EIRP}_{T X}+G_{R}-L_{R X}-L_{P}-L_{\mathrm{atmo}}-L_{\mathrm{iono}},
$$


with the atmospheric and ionospheric losses each estimated at $1 \mathrm{~dB}[26]$.

\subsubsection{Carrier-to-Noise Ratio}

The carrier-to-noise ratio is the ratio of carrier power to the noise power at the receiver [25]. The carrier power is equivalent to the received power calculated in Section 3.5.1. To determine the noise power,

$$
P_{n}[\mathrm{dBW}]=k+T_{s y s}+B_{n}
$$

where the $k$ is Boltzmann's constant, $T_{S}$ is the system temperature calculated in Section 3.4.4, and $B_{n}$ is the noise bandwidth. $B_{n}$ is equivalent to the system symbol rate, as root raised cosine filtering is assumed at the receiver [27].

Given the received power (3.11) and noise power (3.12), the carrier-to-noise ratio is calculated by

$$
\mathrm{CNR}[\mathrm{dB}]=P_{R}-P_{n}
$$

Finally, in order to perform LA analysis as a function of bit error rates in the following section, the carrier-to-noise ratio is related to the received energy per symbol through

$$
\frac{E_{s}}{N_{0}}=\frac{C T_{s} B_{n}}{N} .
$$

Given that the noise bandwidth $B_{n}$ is equivalent to the symbol rate $R_{S}$ and the symbol 


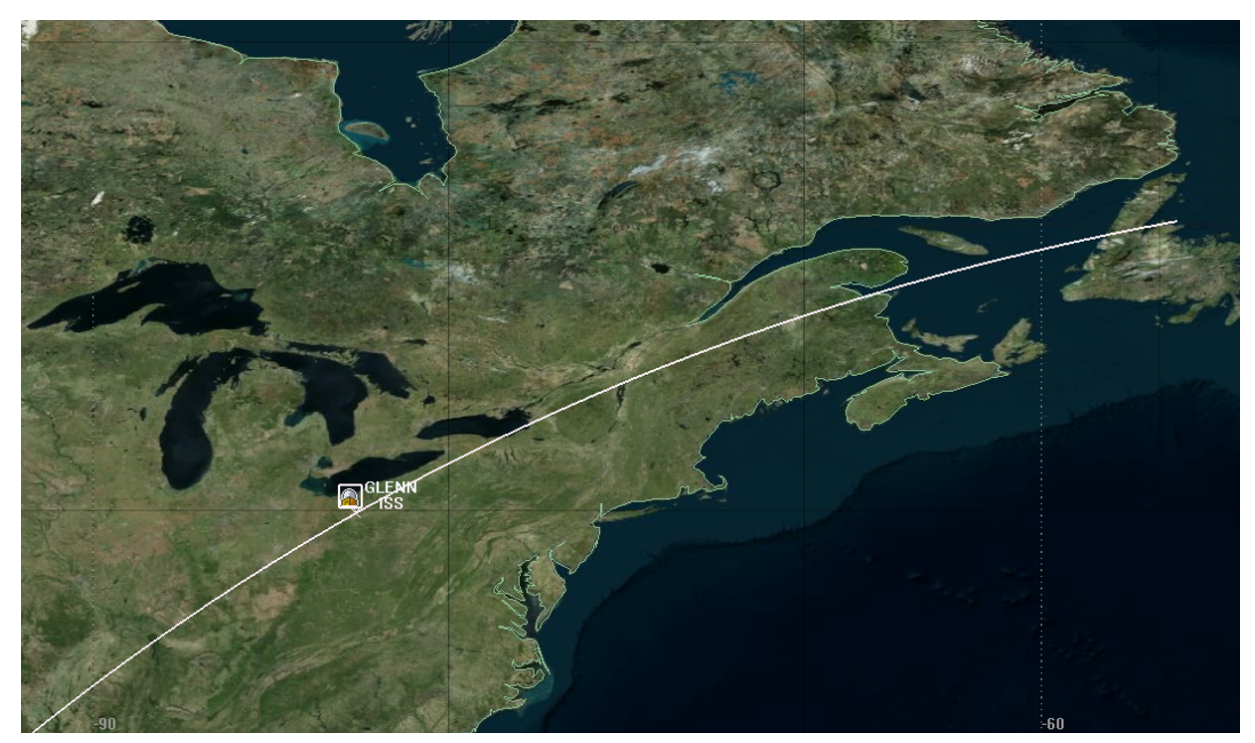

Figure 3.9: Ground track of best-case link budget.

period $T_{S}$ is the reciprocal of the symbol rate, (3.14) simplifies to

$$
\frac{E_{s}}{N_{0}}=\frac{C}{N}
$$

\subsubsection{Best-Case Link Budget}

To understand the full range of carrier-to-noise ratios experienced at the receiver, both a best-case and worst case link budget calculation was performed prior to consideration of any solar-panel obstruction of the receiving antenna. The best-case path loss value of $151 \mathrm{~dB}$ occurs during the scenario when the ISS is at a range of $407.1881 \mathrm{~km}$. This range correlates to the point during the ISS' orbit when it is directly over the ground station. Figure 3.9 outlines the ground track for the entire access period, and shows the point in the orbit that has the lowest path loss. The elevation and azimuth of the ISS at the midpoint of the pass 
Table 3.2: Best Case Link Budget

\begin{tabular}{lr}
\hline Received Power & \\
\hline TX Antenna Gain $(\mathrm{dB})$ & 32.5 \\
TX Power, 10 W (dB) & 10 \\
TX Losses (dB) & 2 \\
Path Loss (dB) & 151 \\
Atmospheric Losses (dB) & 1 \\
Ionospheric Losses (dB) & 1 \\
Rx Antenna Gain $(\mathrm{dB})$ & 1.8 \\
Received Power (dBW) & -110.7 \\
\hline Uplink Noise Power & \\
\hline $\mathrm{k}$, Boltzmanns Constant $(\mathrm{dBW} /(\mathrm{dBK} / \mathrm{Hz})$ & -228.6 \\
System Temperature, $632.5 \mathrm{~K}(\mathrm{dBK})$ & 28 \\
Noise Bandwidth, $502 \mathrm{kHz}(\mathrm{dBHz})$ & 57 \\
Receiver Noise Power $(\mathrm{dBW} / \mathrm{K} / \mathrm{Hz})$ & -140.6 \\
\hline Carrier-to-Noise Ratio $(\mathrm{dB})$ & 32.9 \\
\hline
\end{tabular}

(when directly overhead of the ground station) correspond to a receiver antenna gain of 1.8 dBi. The duration of the pass is 10.7 minutes.

Table 3.2 outlines the full link-budget analysis for the best case pass scenario, before any solar panel obstruction is taken into account. The $\mathrm{C} / \mathrm{N}$ is $32.9 \mathrm{~dB}$.

\subsubsection{Worst-Case Link Budget}

The worst case path loss occurs when the ISS is at a range of $2336 \mathrm{~km}$ from the ground station. This range correlates to the ISS position in orbit when furthest away from the ground station while still visible. Figure 3.10 shows the point during the orbit with the largest path loss; note that at the midpoint of the pass, the ISS is not directly overhead like 


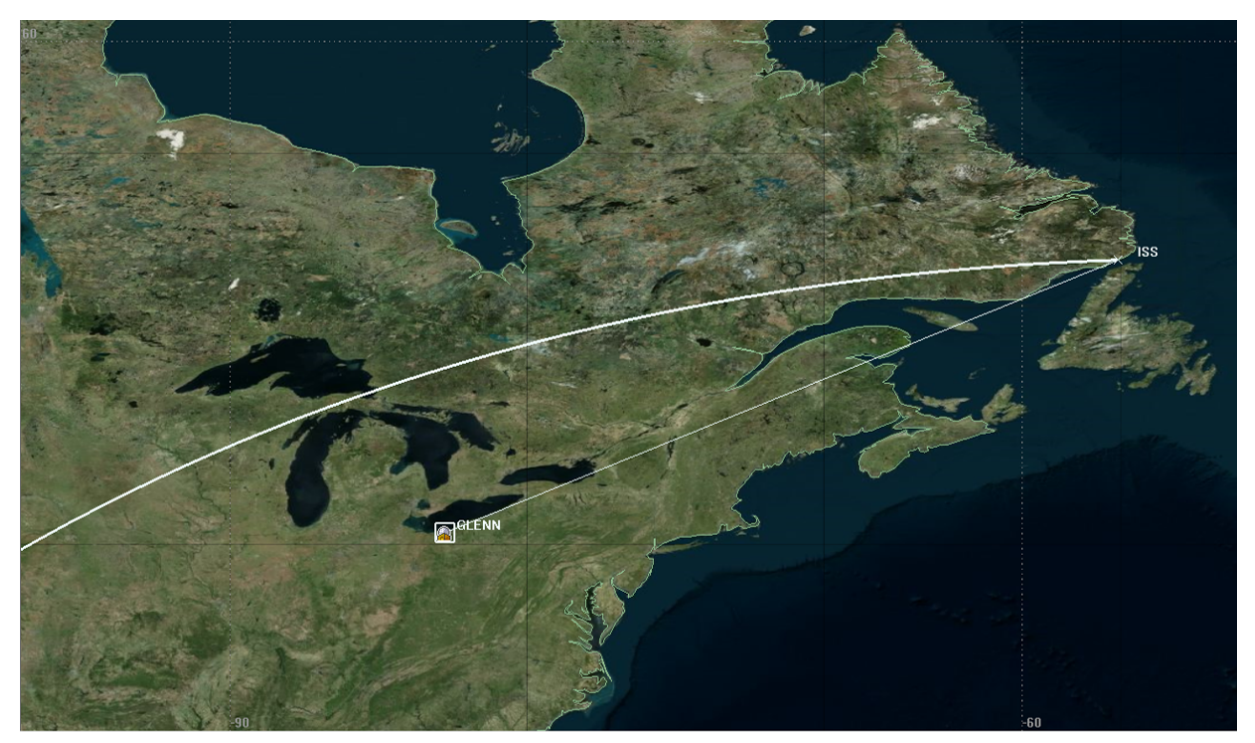

Figure 3.10: Ground track of worst-case link budget.

in Fig. 3.9. The elevation and azimuth of the testbed corresponds to a receiver antenna gain of $2.8 \mathrm{dBi}$. The worst case path loss is $166.1 \mathrm{~dB}$.

Table 3.3 outlines the full link-budget analysis for the worst case pass scenario, before any solar panel obstruction is taken into account. The resulting $\mathrm{C} / \mathrm{N}$ is $18.8 \mathrm{~dB}$.

\subsection{Doppler Shift}

Doppler shift is another time-varying parameter that needs to be addressed in the uplink. While this consideration does not need to be included in the overall link budget analysis, it does need to be addressed in order to avoid frequency offsets. Because Doppler shift can be calculated before a link is established with the satellite, it is assumed here that the ground station transmitter will adjust for the shift as communication begins. This method 
Table 3.3: Worst Case Link Budget

\begin{tabular}{lr}
\hline Received Power & \\
\hline TX Antenna Gain $(\mathrm{dB})$ & 32.5 \\
TX Power, 10 W (dB) & 10 \\
TX Losses (dB) & 2 \\
Path Loss (dB) & 166.1 \\
Atmospheric Losses (dB) & 1 \\
Ionospheric Losses (dB) & 1 \\
Rx Antenna Gain $(\mathrm{dB})$ & 2.8 \\
Received Power (dBW) & -124.8 \\
\hline Uplink Noise Power & \\
\hline $\mathrm{k}$, Boltzmanns Constant $(\mathrm{dBW} /(\mathrm{dBK} / \mathrm{Hz})$ & -228.6 \\
System Temperature, $632.5 \mathrm{~K}(\mathrm{dBK})$ & 28 \\
Noise Bandwidth, $502 \mathrm{kHz}(\mathrm{dBHz})$ & 57 \\
Receiver Noise Power $(\mathrm{dBW} / \mathrm{K} / \mathrm{Hz})$ & -140.6 \\
\hline Carrier-to-Noise Ratio $(\mathrm{dB})$ & 18.8 \\
\hline
\end{tabular}

of adjustment is common among amateur radio operators [19]. Consideration for Doppler at the ground station will relax the design requirements for the flight SDR. The Doppler shift will be calculated before the pass begins using the range rate component from STK. STK returns range rate for each time step in the satellite pass allowing the Doppler shift to be calculated by

$$
\Delta f=\left(\frac{v_{r}}{c}\right) f_{0},
$$

where $v_{r}$ is the range rate $(\mathrm{m} / \mathrm{s})$ of the ISS. The values in Table 3.4 outline the maximum and minimum Doppler shift experienced by the satellite.

The uplink Doppler adjustment will be performed by the ground station. Thus, the frequency offsets must account for the Doppler from the perspective of the ISS. For example, 
Table 3.4: Summary of Doppler Frequency Characteristics

\begin{tabular}{llll}
\hline \multicolumn{2}{l}{ Doppler Frequency } & & \\
\hline & & Minimum & Maximum \\
$v_{r}$ & Velocity of Receiver & $-6.8 \mathrm{~km} / \mathrm{sec}$ & $+6.8 \mathrm{~km} / \mathrm{sec}$ \\
$\Delta f$ & Doppler Frequency & $-50 \mathrm{kHz}$ & $+50 \mathrm{kHz}$ \\
\hline
\end{tabular}

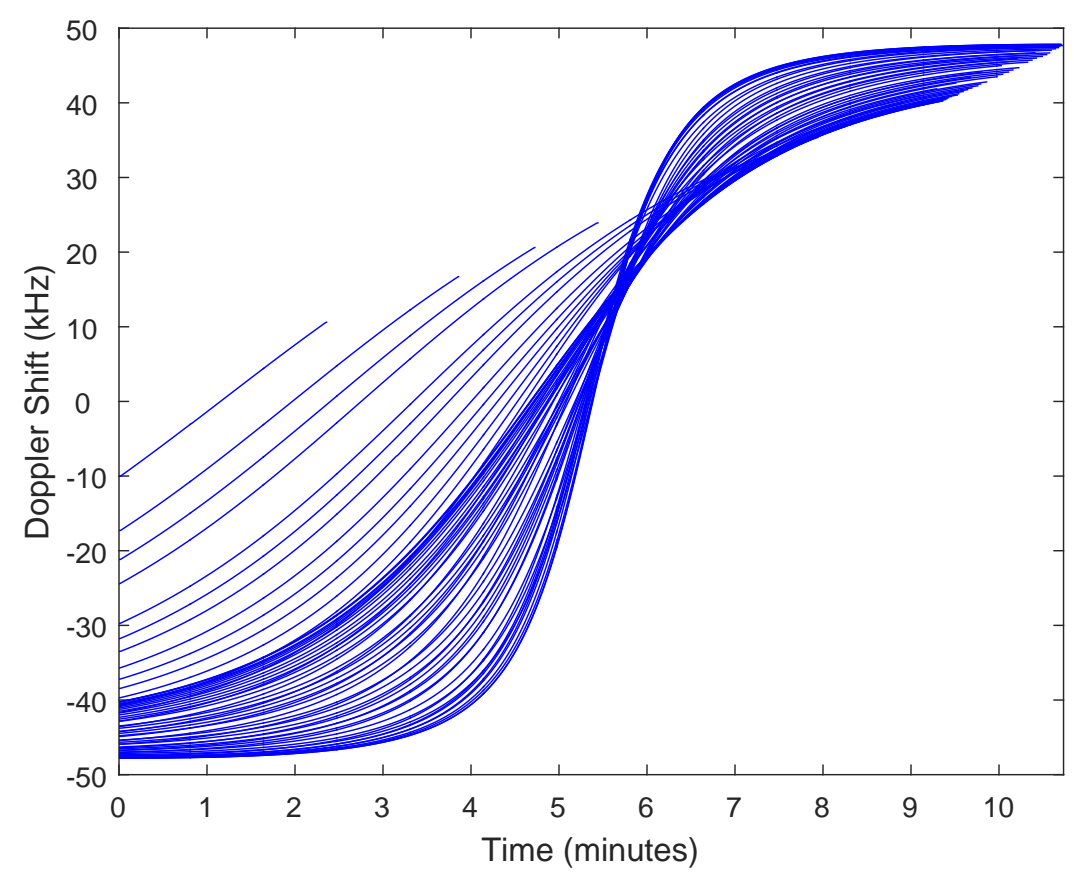

Figure 3.11: Time-varying Doppler shift for 60 accesses.

as the ISS is traveling towards the ground station, the frequency observed at the ISS is higher than the transmitted frequency. As a result, at GRC, the transmitter will intentionally offset the frequency to $f_{0}-\Delta f$. Figure 3.11 displays the Doppler frequencies for 60 accesses, and thus, the calculated frequency offsets that must be applied at the ground station in order to adjust for the Doppler shift. 


\section{Chapter 4}

\section{Link Adaptation Algorithm}

This chapter discusses a link adaptation algorithm approach that aims to move away from the worst-case assumption discussed in Chapter 1 and instead adapt the transmitter modulation schemes to the link conditions, modeled in Chapter 3, to maximize data throughput while continuing to maintain low BER. First, a survey of link adaptation approaches and applications is presented in Section 4.1. Section 4.2 explores a method for decision-making, considering bit error rate, signal-to-noise ratio, and error vector magnitude. Upon identifying the decision metric, a full algorithm model and performance analysis is presented in Section 4.3.

\subsection{Prior Work}

Link adaptation is a broad term for a technique that monitors a communications link and alters system parameters to meet certain conditions. There are a few different methods of link adaptation traditionally recognized. Adaptive Power Application (APA) is the method 
in which the transmitted power is adjusted [3,28]. Adaptive Coding and/or Modulation $(\mathrm{ACM})$ is the approach in which the transmitted modulation and/or coding schemes are altered $[5,6,29]$. ACM is also referred to as rate adaptation, as the data rate of the link does vary as modulation and coding schemes change.

All LA techniques require channel quality heuristics in order to determine when adaptation should take place to achieve a goal. Common quality metrics characterize the link conditions, such as signal-to-interference-plus-noise ratio (SINR), signal-to-interference ratio (SIR), and signal-to-noise ratio (SNR). These metrics are monitored and adapted to optimize a pre-selected parameter such as data throughput, coverage area, bit error rate, energy efficiency, etc..

LA has been applied in cellular networks like GSM, EDGE (TDMA), and CDMA [3, $5,28,29]$. Cellular systems have a minimum data rate threshold that must be provided in the coverage area. However, in areas of higher SINR, the data rate of the system can be increased, thus improving the spectral efficiency in that area. For CDMA, pilot symbols are used to estimate SINR with link adaptation comprised of both ACM and variable spreading [28]. Additionally, the Near-Far problem seen in CDMA systems is addressed by adapting the power in the handsets to adjust the range from the base station [30]. TDMA leverages ACM, while estimating frame error rate (FER) and average SINR at the receiver [28]. Rather than estimating the carrier-to-interference ratio (CIR) at the receiver, soft output average and errors in training sequence have been suggested as methods to estimate BER in GSM and thus trigger switching from the BER estimate [29].

An application for LA has also been presented for Wireless LAN, with a focus on 
maximizing data throughput through rate adaptation $[6,31]$. The channel quality of the link is monitored and should it improve, the frame size is altered using an optimal size predictor to maximize the throughput [6]. Additionally, another application of LA in WiFi systems focuses on maximizing energy efficiency in long-distance WiFi links by using a combination of APA and rate adaptation [32].

Link adaptation is discussed for application in cooperative communication networks, in which wireless nodes work together to transmit information [33,34]. ACM is combined in conjunction with relay adaptation to limit effects of time-varying fading channels and exploit favorable conditions to maximize data throughput [33-35].

More targeted applications for LA exist as well. For example, LA has been proposed as a tool to improve physical layer security in wireless links [36]. Additionally, link adaptation to maximize energy efficiency has been proposed for wireless body area networks (WBAN) [37]. As WBAN sensors are small and embedded within the body, efficient use of the battery is a requirement and thus makes them a practical area for LA.

LA has also been suggested as a tool to leverage availability of Television White Space (TVWS) channels through channel aggregation with ACM [38]. Similarly, investigation into LA in a dynamic spectrum access application for cognitive radios has also been presented [39]. LA in Land Mobile Satellite systems have also been proposed and analyzed [7, 8]. With mobile users, the channel conditions can vary greatly during the feedback delay. SNR can be reported during slow-fading conditions but, should the channel change quickly, the LMS LA approach would not adjust in time [8]. This effect is also seen in cellular LA 
systems, as a slow-fading channel is assumed so that SIR estimates can be obtained [5,28]. Some investigation into LA on fast-fading channels has been made, largely in high-speed railways and vehicle-to-vehicle communications [40-44]. However, for the purposes of this link adaptation algorithm, slow-fading channels across measurements and estimations is assumed.

All the above applications of LA are Earth-based; however, instances of LA in satellite communications exist as well. Resources and channels are dynamically allocated to users based upon IP packets waiting to be transmitted on each channel, thus maximizing throughput $[9,45]$. This dynamic allocation allows for the terminal with higher link quality to be assigned more resources. This approach has been investigated for TDMA and OFDM on military satellites in GEO orbit.

A channel estimation and ACM approach for $\mathrm{Ku}$ - and Ka-band satellite broadband networks is investigated [13]. As rain fade significantly affects systems at Ku-band and Ka-band, satellite providers must plan a high link margin into the system design [46]. This high link margin can support higher throughput when rain is not present; ACM can maximize the data rate. The channel metric, SINR, is occasionally estimated using pilot symbols.

Similarly, DVB-S2 (Digital Video Broadcasting-2nd Generation Satellite) supports ACM to optimize data transmission for unicast commercial and professional services [47]. The transition from DVB-S to DVB-S2 brought around a 30\% spectrum efficiency improvement [48]. There have been investigations into applying ACM to broadcasting streams such as H.264 video services [48]. The channel-quality feedback from each user in unicast transmission 
is easily applied to an ACM algorithm. However, in broadcast, channel-quality feedback is not available from all the users who could be receiving. Using a management model to compile status reports is suggested as an option for the feedback loop in broadcasting [48]. Another study claims that ACM could be used with elementary feedback from the gateway station [11].

An ACM system for a future Algerian LEO microsatellite was designed using QAM modulation schemes and Rayleigh and Ricean channel models [12]. However, the study did not address how to measure the channel quality heuristics on-board the satellite.

Unlike most of the satellite LA applications, the system within this work includes feedback from the receiver to the transmitter. The LA algorithm developed in this chapter focuses on maximizing data throughput through adaptive modulation. As seen above, adaptive coding and power adaptation are both other common techniques, and both could be applied to also improve data throughput. For this work, however, the focus on one adaptation method minimizes the complexity of the receiver and algorithm on the hardware. The following sections outline the design approach and modeling of the adaptive modulation algorithm.

\subsection{Decision Metric}

As discussed in Section 4.1, LA algorithms adapt based upon channel quality characteristics. Section 4.2 discusses the metrics considered in the following algorithm design, addressing shortcomings and ultimately identifying a method. 


\subsubsection{Bit Error Rate}

At a receiver, the goal is to perfectly reconstruct the transmitted data, reproducing the symbols that were initially transmitted. The receiver must decide which symbol was transmitted from the noisy signals received. The probability of error at the end of this process is an established figure of merit for the communication quality. More specifically, the probability of bit error, also known as bit error rate (BER), is a direct measurement of the quality of the communications link [49].

In any link adaptation algorithm, a goal must be established to design the adaptation around. For this work, the focus of the adaptation algorithm is to maximize data throughput while staying below a BER threshold. For instance, the objective BER for video transmission is often $10^{-7}$ or $10^{-8}$ and the objective BER for vocoded speech is often $10^{-2}$ or $10^{-3}$ [25]. For data transmission on wireless channels, a common BER goal is $10^{-5}$ or $10^{-6}$ [25]. As data transmission to the flight SDR is the main objective in this work, a BER threshold of $10^{-5}$ is selected, although the threshold can be changed depending on the application of the system.

On-board the SCaN receiver, the BER must be measured or estimated to monitor the current error rate of the data. For this work, the BER will be estimated. If the BER is measured directly, the transmitted data must be known at the receiver in order to identify a bit error. A pilot sequence of known bits must be transmitted in order to calculate the BER. Additionally, in order to have an accurate measurement, it is assumed 100 bits need 
Table 4.1: Average Time to Measure BER for each Modulation Scheme

\begin{tabular}{lr}
\hline \multicolumn{2}{c}{ Average Time to Receive 100 Errors } \\
\hline BPSK & $19.92 \mathrm{~s}$ \\
QPSK & $9.96 \mathrm{~s}$ \\
8PSK & $6.64 \mathrm{~s}$ \\
16QAM & $4.98 \mathrm{~s}$ \\
64QAM & $3.32 \mathrm{~s}$ \\
\hline
\end{tabular}

to be measured "in error". With a BER threshold of $10^{-5}$, the receiver must receive on average 100,000 bits before one is in error. Therefore, an average of 10,000,000 bits must be received for 100 bits to be in error. As defined in Chapter 2, all the modulation schemes are transmitted at a fixed symbol rate of $502 \mathrm{kSps}$, so at the lowest bit rate, the receiver would require roughly 19.9 seconds to measure the BER. Table 4.1 summarizes the time necessary for each modulation scheme to receive 100 errors, assuming a BER threshold of $10^{-5}$.

The longest pass over the ground station is 10.7 minutes, and the shortest is 12 seconds. To allocate at most 20 seconds to measure BER for every mode switch would limit the data transmission, as pilot sequences would be needed. Additionally, the channel changes more quickly than every 20 seconds. It is possible that by the time the receiver finishes measuring the BER, channel conditions have shifted, causing the measurement to be inaccurate. Thus, while BER is the figure-of-merit used to measure the quality of a communications link, it should not be directly measured in this application. Instead, the decision metric for this algorithm should find a method to estimate BER. 


\subsubsection{Signal-to-Noise Ratio}

Given an AWGN channel, the BER can be written as a function of the $Q$-function and $E_{b} / N_{0}$. The $Q$-function is defined as [18]

$$
Q(x)=\frac{1}{\sqrt{2 \pi}} \int_{x}^{\infty} e^{-\frac{t^{2}}{2}} d t,
$$

and $E_{b} / N_{0}$ is defined as the ratio of modulated energy per information bit to the noise spectral density [25]. $E_{b} / N_{0}$ is commonly seen as the metric used to calculate bit error rates of modulation schemes $[18,25,49]$. Referring back to $(3.14), E_{b} / N_{0}$ is related to the signal-to-noise ratio in digital systems as:

$$
\mathrm{SNR}=\frac{E_{S}}{N_{0}}
$$

Gray coding of all modulation schemes is assumed, so as to keep each neighboring symbol to differ by only one bit. This intentional symbol permutation decreases the likelihood of more than one bit being in error at any one time [25]. See Appendix A for the specific Gray coding arrangement for each modulation scheme.

Using $E_{b} / N_{0}$ to calculate BER, BPSK and QPSK have the same bit error rate probability, as follows

$$
P_{b}=Q\left(\sqrt{\frac{2 E_{b}}{N_{0}}}\right)
$$

Thus, when comparing the performance of BPSK and QPSK using $E_{b} / N_{0}$, it appears as 
if the required energy to maintain the same $P_{b}$ is identical. If, however, the BER were to be written in terms of the modulated energy per symbol, a $3 \mathrm{~dB}$ increase of signal energy is evident. This $3 \mathrm{~dB}$ is generated by doubling the energy used in the modulation constellation, as a result of using both the quadrature and in-phase channels rather than just in-phase of BPSK. $E_{b} / N_{0}$ is related to $E_{S} / N_{0}$ through

$$
\frac{E_{b}}{N_{0}}=\frac{1}{\log _{2} M} \frac{E_{S}}{N_{0}},
$$

where $M$ is the level of modulation and $\log _{2} M$ is the number of bits per symbol. From (4.4), the probability of bit errors for BPSK and QPSK are as follows:

$$
P_{b, \mathrm{BPSK}}=Q\left(\sqrt{\frac{2 E_{S}}{N_{0}}}\right) \quad P_{b, \mathrm{QPSK}}=Q\left(\sqrt{\frac{E_{S}}{N_{0}}}\right)
$$

In order to identify the received symbol power necessary in QPSK to achieve a desired BER, the BER calculations, plots, and analysis are all performed with $E_{S} / N_{0}$. Table 4.2 displays the equations used for all five modulation schemes analyzed in this algorithm, where $\mathrm{M}$ is the level of modulation [18]. Figure 4.1 demonstrates these equations plotted across a range of $E_{S} / N_{0}$ from 0 to $35 \mathrm{~dB}$.

The curves given in Table 4.2 allow for a precise calculation of the BER from an $E_{S} / N_{0}$ value. Chapter 3 showed that the link budget returns a value of $E_{S} / N_{0}$, after incorporating all propagation effects. Figure 4.2 shows one example of the increase and decrease in $E_{S} / N_{0}$ as the ISS moves through an access. All the $E_{S} / N_{0}$ values seen in Fig. 4.2 were used in 


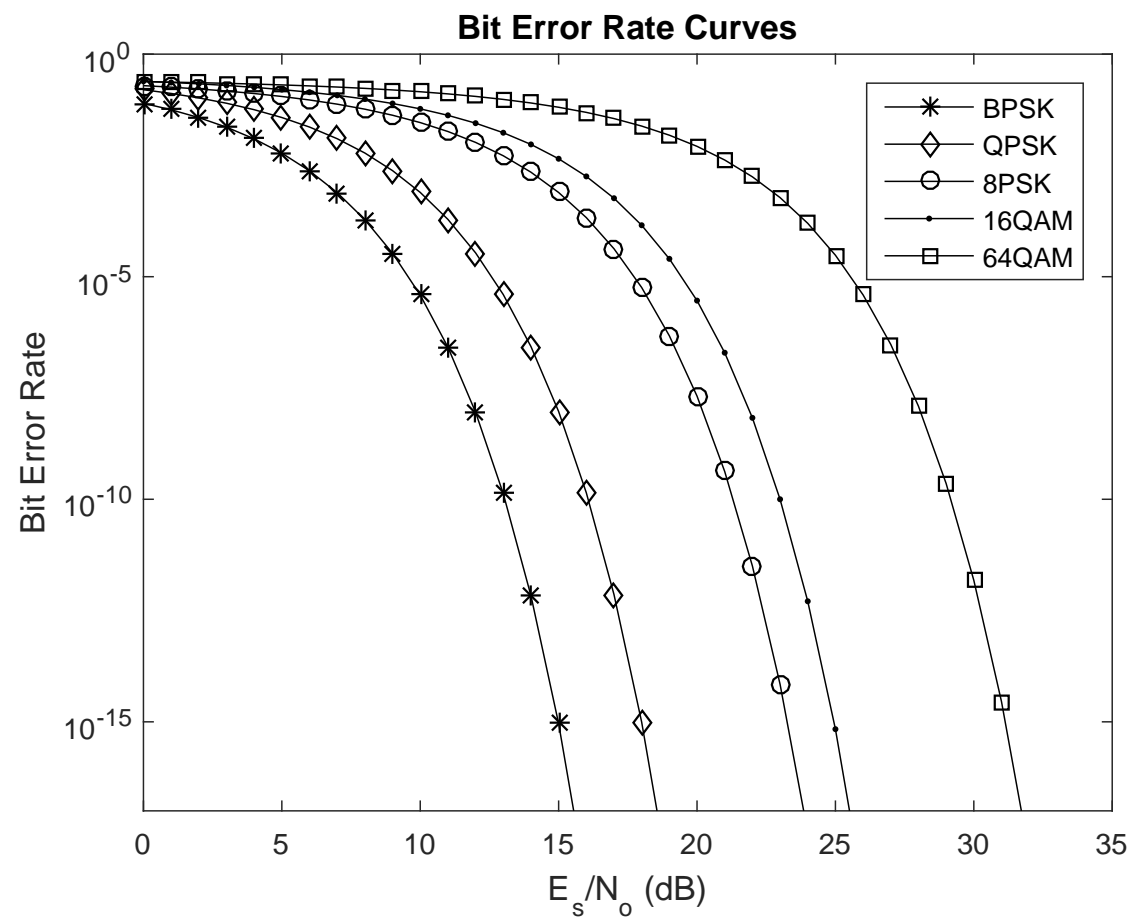

Figure 4.1: Bit error rate curves for analyzed modulation schemes.

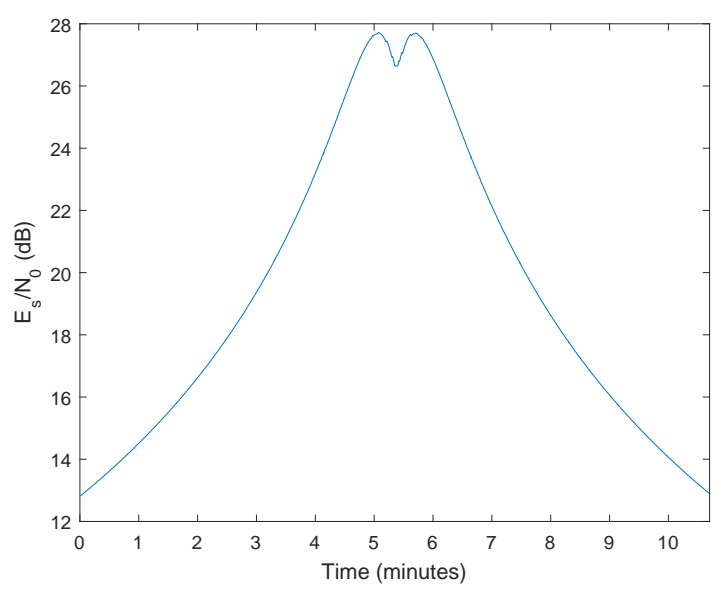

Figure 4.2: $E_{s} / N_{0}$ of one access.

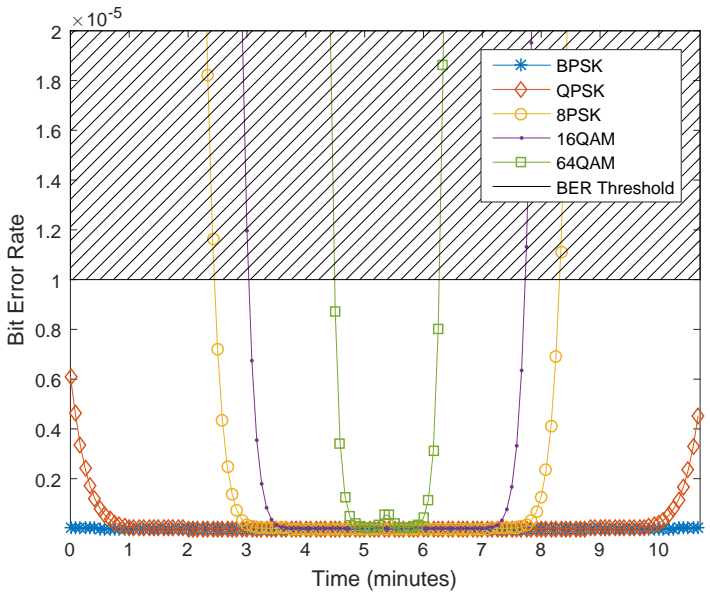

Figure 4.3: BER calculations for one access. 
Table 4.2: Theoretical Bit Error Rate Equations

\begin{tabular}{ll}
\hline BPSK & $P_{b}=Q\left(\sqrt{\frac{2 E_{s}}{N_{0}}}\right)$ \\
MPSK & $P_{b}=\frac{2}{\log _{2} M} Q\left(\sqrt{\left(\sin \frac{\pi}{M}\right)^{2} \frac{E_{s}}{N_{0}}}\right)$ \\
MQAM & $P_{b}=\frac{4}{\log _{2} M}\left(1-\frac{1}{\sqrt{M}}\right) Q\left(\sqrt{\frac{3}{M-1} \frac{E_{s}}{N_{0}}}\right)$ \\
\hline
\end{tabular}

Table 4.3: Minimum $E_{s} / N_{0}$ Necessary to Achieve Selected BER Thresholds

\begin{tabular}{lrrr}
\hline & \multicolumn{1}{c}{$10^{-2}$} & \multicolumn{1}{c}{$10^{-5}$} & $10^{-7}$ \\
\hline BPSK & $4.4 \mathrm{~dB}$ & $9.6 \mathrm{~dB}$ & $11.4 \mathrm{~dB}$ \\
QPSK & $7.4 \mathrm{~dB}$ & $12.6 \mathrm{~dB}$ & $14.4 \mathrm{~dB}$ \\
8PSK & $12.1 \mathrm{~dB}$ & $17.8 \mathrm{~dB}$ & $19.6 \mathrm{~dB}$ \\
16QAM & $14.0 \mathrm{~dB}$ & $19.5 \mathrm{~dB}$ & $21.3 \mathrm{~dB}$ \\
64QAM & $19.8 \mathrm{~dB}$ & $25.6 \mathrm{~dB}$ & $27.4 \mathrm{~dB}$ \\
\hline
\end{tabular}

the equations from Table 4.2. The resulting five BER curves are shown in Fig. 4.3. The dashed shaded area shows the region that the BER threshold $\left(10^{-5}\right)$ is not satisfied. Observe that both BPSK and QPSK meet the BER requirement for for the entire pass duration; meanwhile, 8PSK, 16QAM, and 64QAM all achieve the threshold for shorter periods of time. This result is to be expected as the higher order modulation schemes require stronger symbol energy to achieve the same BER.

Looking at the specific values of $E_{S} / N_{0}$ when each modulation scheme achieves the BER threshold is important for modeling adaptive modulation. Table 4.3 summarizes the minimum value of $E_{S} / N_{0}$ necessary in order to achieve the BER threshold. The table includes a BER threshold for vocoded, data transmission over wireless channels, and video broadcasting. 
Table 4.4: $E_{s} / N_{0}$ Upper and Lower Boundaries for a BER of $10^{-5}$

\begin{tabular}{lrr}
\hline & DOWN & \multicolumn{1}{c}{ UP } \\
\hline BPSK & $\mathrm{N} / \mathrm{A}$ & $13.6 \mathrm{~dB}$ \\
QPSK & $12.6 \mathrm{~dB}$ & $18.8 \mathrm{~dB}$ \\
8PSK & $17.8 \mathrm{~dB}$ & $20.5 \mathrm{~dB}$ \\
16QAM & $19.5 \mathrm{~dB}$ & $26.6 \mathrm{~dB}$ \\
64QAM & $25.6 \mathrm{~dB}$ & $\mathrm{~N} / \mathrm{A}$ \\
\hline
\end{tabular}

The $E_{S} / N_{0}$ characterization seen in Fig. 4.2, does not always have a linearly, parabolic-like received power characterization during every pass. While the pass loss itself provides a predictable link budget result, the solar panel shadowing is introduced suddenly and can cause a dramatic drop in received signal power. Because the received power is subject to sudden fluctuations, the bounds for switching modulation modes must incorporate a hysteresis period. The purpose of the hysteresis period is to prevent the receiver from oscillating between modes should $E_{S} / N_{0}$ hover on the cusp of a threshold. Oscillating modes would not be conducive to continuous data transmission, as each time the receiver switches, the feedback must be transmitted to the ground, which introduces delay.

To design the most effective hysteresis region, an understanding of the scenarios causing the receiver to switch must be explored. There are two situations:

1. Decreasing $E_{S} / N_{0}$ : Receiver will switch from a higher to a lower modulation mode.

2. Increasing $E_{S} / N_{0}$ : Receiver will switch from a lower to a higher modulation mode.

In the first situation, waiting to ensure that the current $E_{S} / N_{0}$ falls within the lower modulation region would cause the bit error rate of the higher order to rise above the 


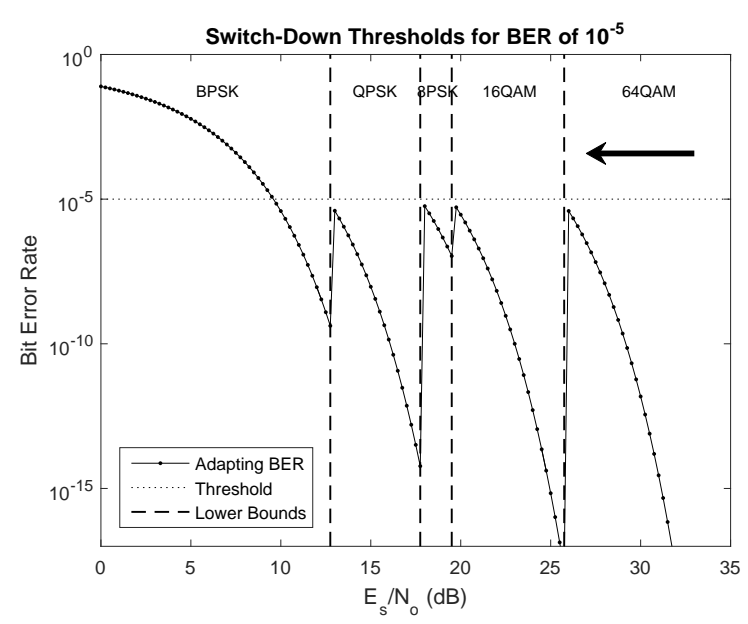

(a) Receiver switches down modulation.

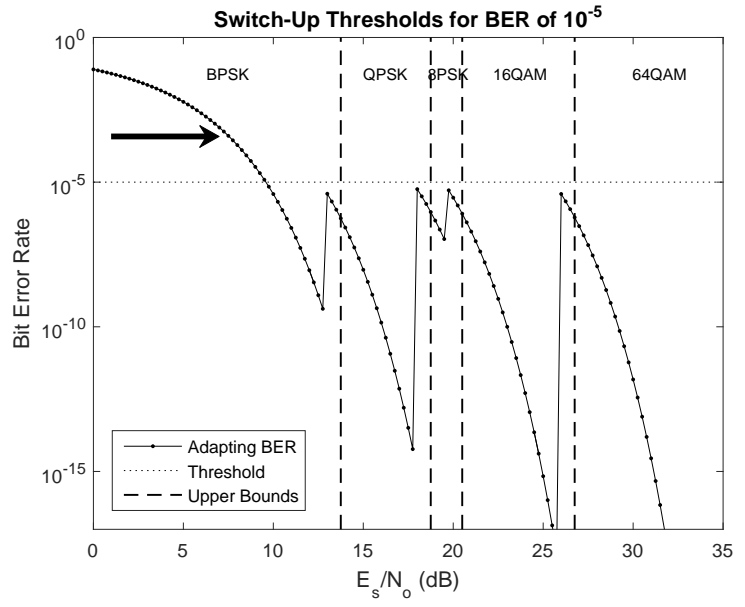

(b) Receiver switches up modulation.

Figure 4.4: Switching thresholds for SNR.

threshold. As the objective of this algorithm is to maintain a BER threshold, this consequence is not desired. The second situation, however, is more conducive for a hysteresis region. As the $E_{S} / N_{o}$ increases, the BER of the current (lower-order) modulation scheme continues to decrease. Thus, waiting to ensure that the current channel conditions can support the next modulation level keeps the BER below the threshold. To define the hysteresis region, a $1 \mathrm{~dB}$ window was applied. The value $1 \mathrm{~dB}$ is used because the 8 PSK region is $2 \mathrm{~dB}$ wide between the 8PSK and 16QAM thresholds. The $1 \mathrm{~dB}$ guard region is applied to the thresholds to switch to a higher modulation order. Table 4.4 outlines the thresholds for switching up and down. As BPSK and 64QAM are the lowest and highest achievable modulation schemes, they don't have a switch-down or switch-up threshold, respectively.

Figure 4.4 displays the operation for both switching scenarios. Decreasing $E_{S} / N_{0}$ (Fig. 
4.4a) will reach the absolute bounds originally introduced in Table 4.3. These thresholds are the lowest possible $E_{S} / N_{0}$ that can return the BER threshold. Alternatively, for increasing $E_{S} / N_{0}$ (Fig. 4.4b), the $1 \mathrm{~dB}$ guard region is applied so that the BER continues decreasing well below the BER threshold until the next modulation scheme can begin operating.

\subsubsection{SNR Measurement}

Ultimately, $E_{S} / N_{0}$ can be related to BER and used as the decision metric for the LA algorithm. In order to use $E_{S} / N_{0}$ to trigger adaptation, the signal-to-noise ratio (SNR) must be known at the receiver, which is unfortunately not the case and thus must be measured or estimated. Most SNR estimators fall into two categories: data-aided (DA) or non-data-aided (NDA). Many DA estimators use pilot symbols to perform the estimation [50-52]. Data gathered from the pilot symbols can then be used in SNR estimators, such as a Maximum Likelihood estimator [50]. DA estimators also perform better than NDA at lower SNRs [53].

The purpose of this work is to maximize data throughput while maintaining a BER threshold, so employing a DA SNR estimator is not prudent as DA receivers have worse bandwidth efficiency than NDA receivers $[52,54,55]$. Five common SNR estimators are: Split-Symbol Moments Estimator (SSME), ML Estimator, Squared-Signal-to-Noise Variance (SNV) Estimator, (Second and Fourth-Order Moments) $\mathrm{M}_{2} \mathrm{M}_{4}$ Estimator and Signal-to-Variation

Ratio (SVR) Estimator. A comparison of these five estimators for both DA and NDA shows that a NDA-aided receiver performed well in performance analysis [52]. However, 
the estimator used an iterative approach, which can introduce delay and computational burdens. Ultimately, for a NDA SNR estimation, selecting the "best" estimator depends on block length, number of samples available for the estimation, type of modulation, and the range of SNR [52]. Within this work, modulation type changes between varying levels of $M$ in PSK as well as QAM, therefore selecting an SNR estimator that performs well on all modulation schemes can prove challenging. An alternative to an SNR estimator is using another figure-of-merit on the communications link to estimate SNR.

\subsubsection{Error Vector Magnitude}

A signal transmitted in a no-noise environment to a receiver with ideal hardware has all constellation points exactly positioned at the expected locations. In any real system, noise causes the constellation points to shift from the ideal positions. Error Vector Magnitude $(\mathrm{EVM})$ is a quantification of the disparity between the received symbol and the ideal symbol and is a common metric to assess the quality of digitally modulated communication signals $[56,57]$. It can be used as an approach to estimate SNR at the receiver as well [58-63]. There are applications for SNR estimation through EVM measurement in both data-aided ([64]) and non-data-aided $([62,65])$ receivers. EVM was used as a decision statistic for QPSK and 16-QAM modes in a link adaptive system proposed [39]. Additionally, the EVM SNR-estimation approach can be more easily implemented in hardware in comparison to a traditional SNR estimator [58,59].

EVM is measured by calculating the distance between the ideal and received symbol. 


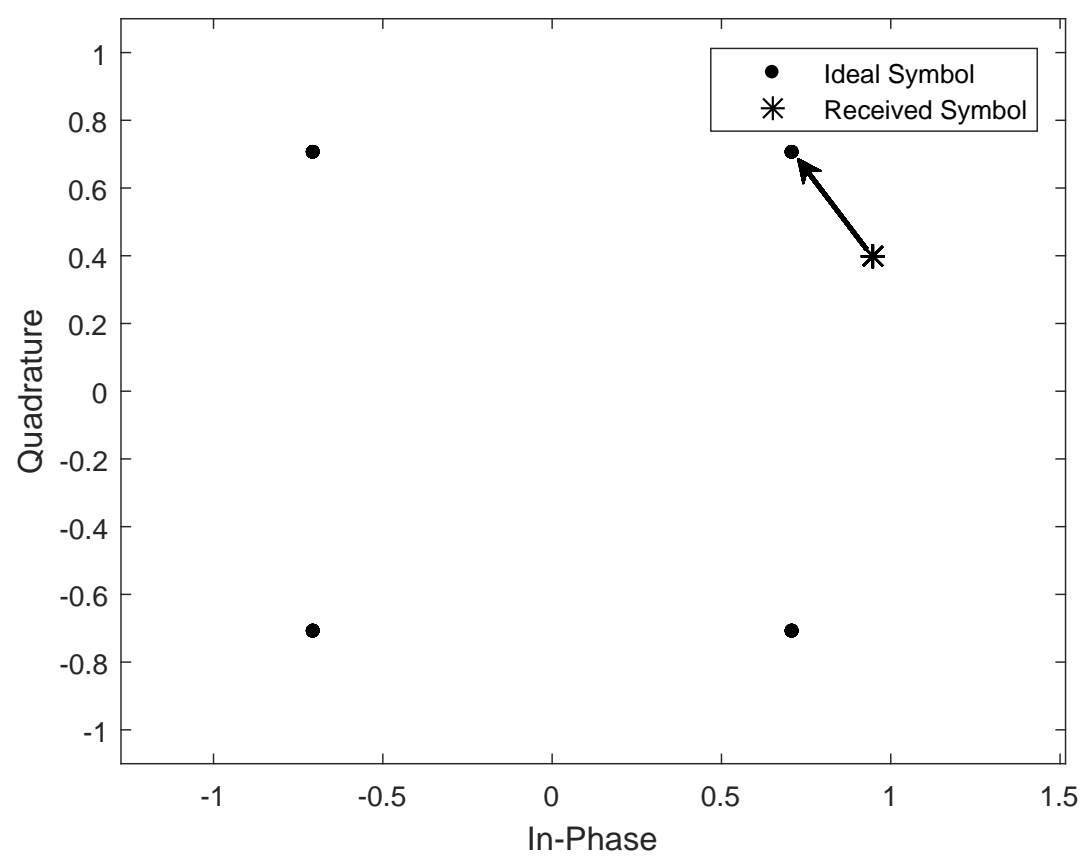

Figure 4.5: Example of QPSK EVM measurement-closest symbol.

This distance is called the "error vector magnitude." The average power of the error vector is then normalized to the signal power [56].

$$
\mathrm{EVM}=\left[\frac{\left|S_{\text {ideal }}-S_{\text {received }}\right|^{2}}{\left|S_{\text {ideal }}\right|^{2}}\right]
$$

Commonly, EVM is defined as the root-mean-square (rms) average of the measurements of $N$ consecutive symbols (4.7):

$$
\operatorname{EVM}_{[\mathrm{rms}]}=\left[\frac{\frac{1}{N} \sum_{r=1}^{N}\left|S_{\text {ideal }}-S_{\text {received }}\right|^{2}}{\frac{1}{N} \sum_{r=1}^{N}\left|S_{\text {ideal }}\right|^{2}}\right]^{\frac{1}{2}}
$$

When calculating EVM, the $S_{\text {ideal }}$ can be the actual transmitted symbol, assuming that 


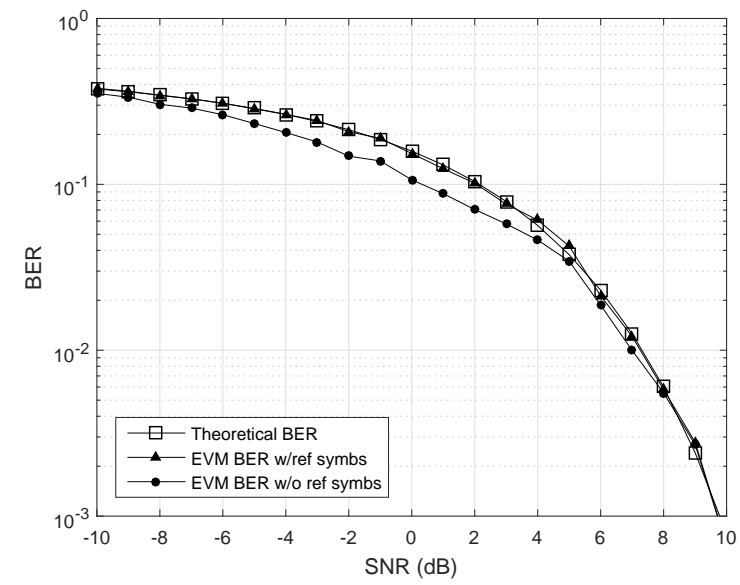

(a) QPSK

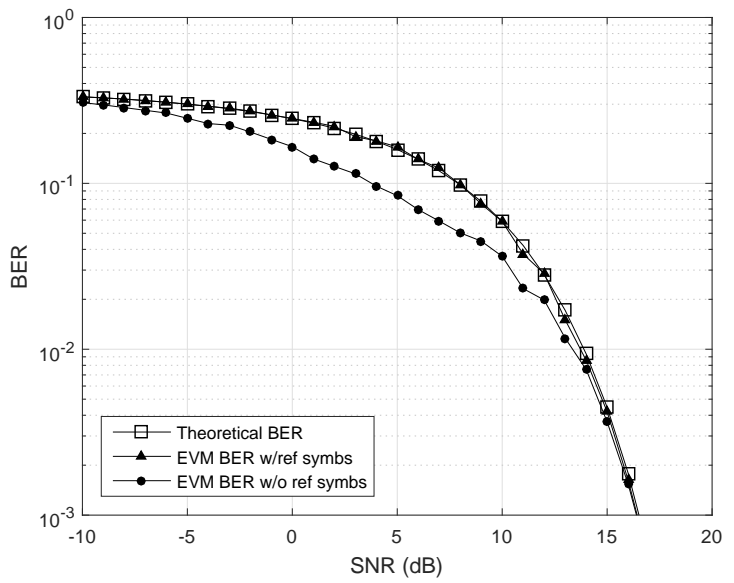

(b) 16QAM

Figure 4.6: Comparison of EVM with and without reference symbols.

it is known at the receiver. However, the receiver on the ISS will not have a priori knowledge of the transmitted symbol stream. Thus, the error vector magnitude calculation will be done by using the symbol closest to the received symbol as $S_{\text {ideal }}$, as shown in Fig. 4.5. MATLAB Simulink contains a block that calculates the EVM of a signal, entitled "EVM Measurement". This block was initially used to verify the EVM calculations done in MATLAB. The block uses the reference signals to calculate the bit error rate, i.e., the block knows what symbol should be received and calculates the distance for that one symbol.

Figure 4.6 shows the BER for QPSK and 16QAM for both EVM-estimated BER, using both reference symbols and then using the closest symbol approach. The number of symbols used in this estimation was 1000. While the EVM BER with reference symbols more closely approximates the theoretical BER, the EVM-estimated BER without reference symbols 


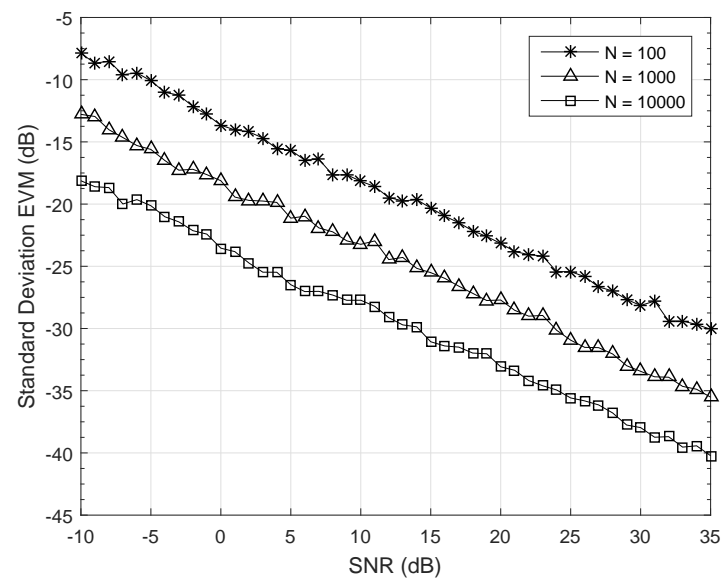

(a) QPSK

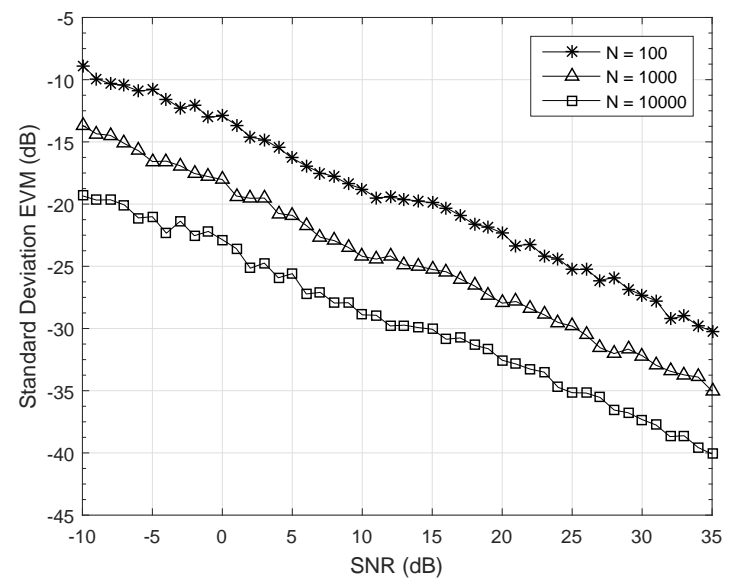

(b) 16QAM

Figure 4.7: Standard deviation of EVM measurements as SNR increases.

matches the theoretical BER as SNR increases.

To use EVM for SNR-estimation, and thus a BER estimation, EVM must be related to SNR. $\mathrm{EVM}_{[\mathrm{rms}]}$ can be related to the signal-to-noise ratio (SNR) [56] when using a large number of symbols, $N$ :

$$
\mathrm{EVM}_{[\mathrm{rms}]}=\sqrt{\frac{1}{\mathrm{SNR}}}
$$

The standard deviation of EVM measurements as SNR increases is shown in Fig. 4.7. As SNR increases, the standard deviation of EVM measurements decreases, as to be expected with the received symbols are closer to the true symbol at high SNR.

As the number of symbols, $N$, used to estimate EVM increases, the mean-squared-error 


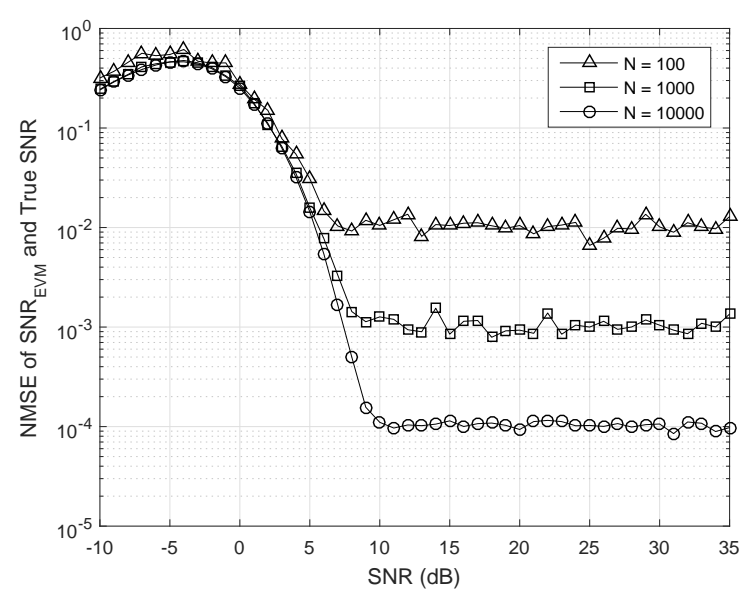

(a) QPSK

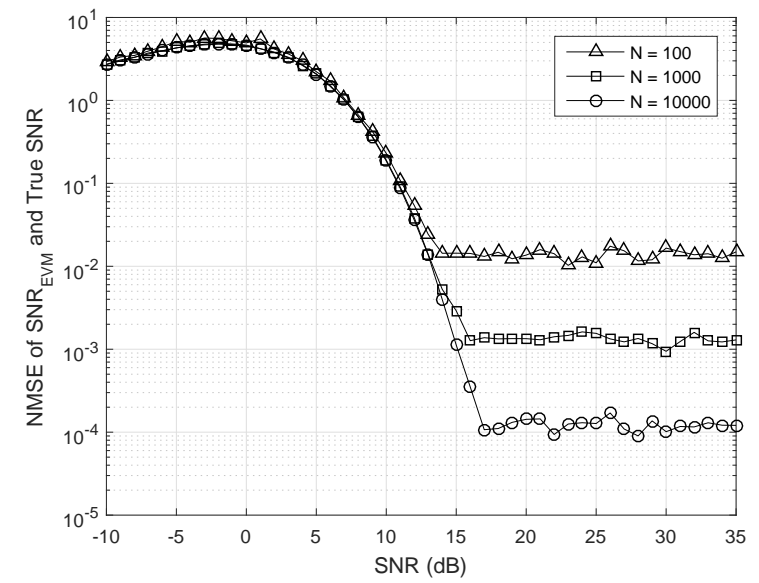

(b) 16QAM

Figure 4.8: Mean-squared error of EVM-estimated SNR and true SNR for varying $N$.

of the EVM-estimated SNR and true SNR approaches zero. Figure 4.8 shows the MSE calculated for varying number of symbols for the QPSK and 16QAM modulation schemes. The EVM-estimated SNR is plotted against the true SNR for both QPSK and 16QAM in Fig. 4.9. The linear relationship between the estimated SNR and true SNR indicates that the EVM-estimation is a close one.

From the SNR-estimation, EVM can then be approximated by $E_{s} / N_{0}$ for a large number of symbols, $N$, as:

$$
\frac{E_{S}}{N_{0}}=\frac{1}{\operatorname{EVM}_{[\mathrm{rms}]}^{2}}
$$

Substituting $\operatorname{EVM}_{[\mathrm{rms}]}$ into the theoretical BER equations in Table 4.2 yields BER estimated by EVM. Table 4.5 shows BER equations with the $E_{S} / N_{0}$ and $\mathrm{EVM}_{[\mathrm{rms}]}$ relationship. 


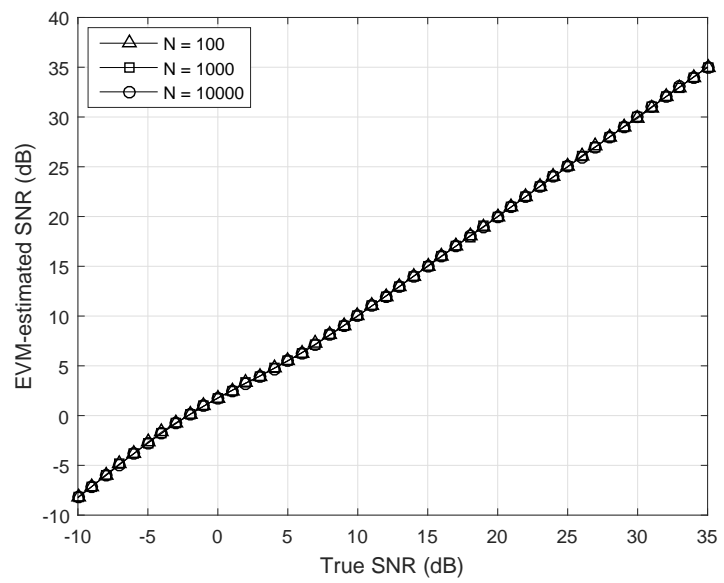

(a) QPSK

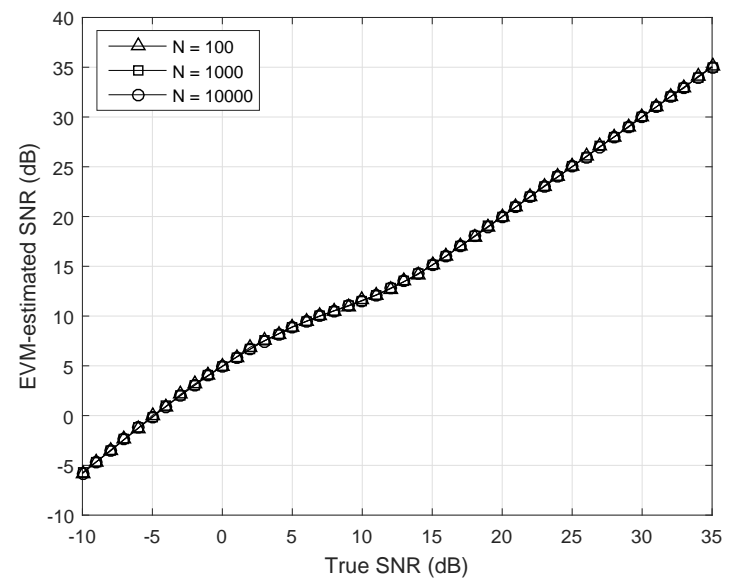

(b) 16QAM

Figure 4.9: Linear relationship between EVM-estimated SNR and true SNR.

Table 4.5: EVM-Estimated Bit Error Rate Equations

\begin{tabular}{ll} 
BPSK & $P_{b}=Q\left(\sqrt{\frac{2}{\mathrm{EVM}_{[\mathrm{rms}]}^{2}}}\right)$ \\
MPSK & $P_{b}=\frac{2}{\log _{2} M} Q\left(\sqrt{\left(\sin \frac{\pi}{M}\right)^{2} \frac{1}{\mathrm{EVM}_{[\mathrm{rms}]}^{2}}}\right)$ \\
MQAM & $P_{b}=\frac{4}{\log _{2} M}\left(1-\frac{1}{\sqrt{M}}\right) Q\left(\sqrt{\frac{3}{M-1} \frac{1}{\mathrm{EVM}_{[\mathrm{rms}]}^{2}}}\right)$ \\
\hline
\end{tabular}

Figure 4.10 compares the EVM-estimated BER for varying number of symbols $N$ to the BER calculated from theory. Observe that with just 100 symbols used in estimation, the EVM-estimated BER closely approximates the theoretical BER. As the number of symbols used in the estimation increases, the error approaches zero-this result indicates that the EVM estimator does not have a bias, and is thus an asymptotically unbiased estimator.

For future simulations, 1000 symbols will be used as the value of $N$, as the MSE between $N$ equal to 1000 and 10000 are almost indistinguishable. Figure 4.11 displays EVM-estimated 


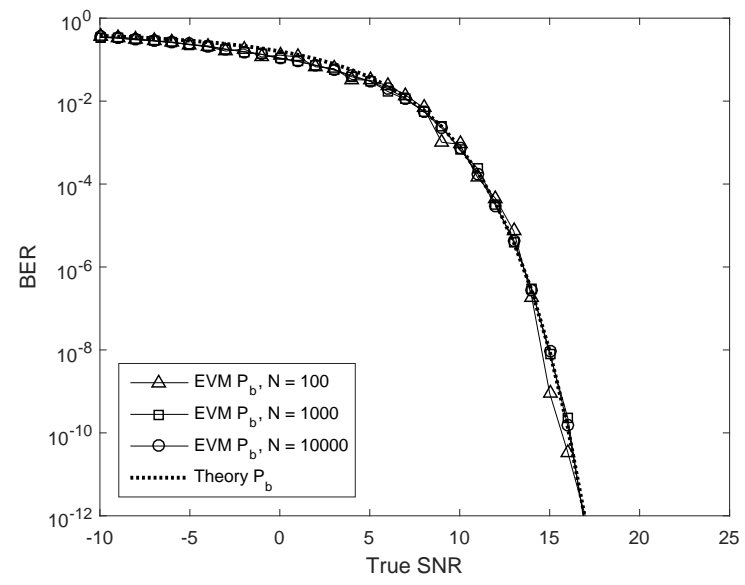

(a) QPSK

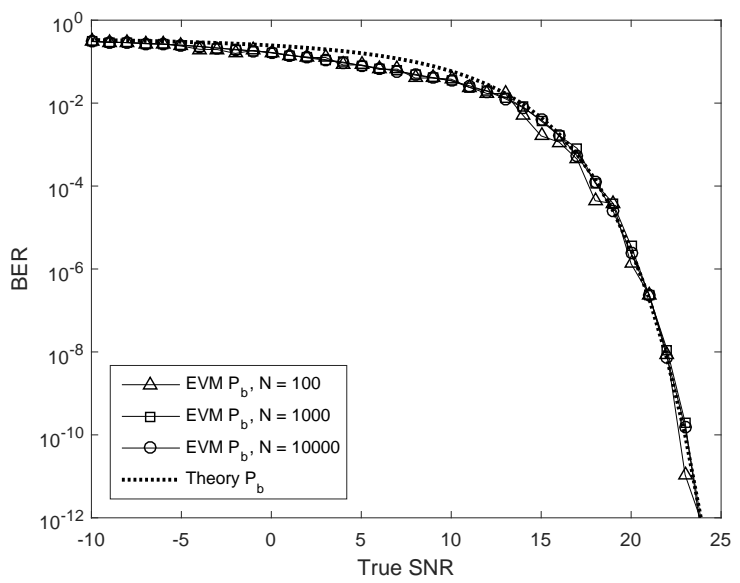

(b) 16QAM

Figure 4.10: Comparison of theory BER and EVM-estimated BER for varying $N$.

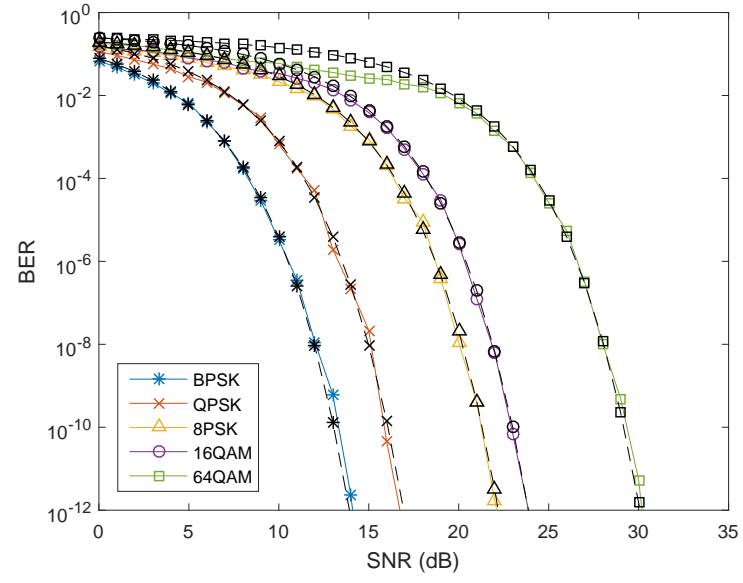

Figure 4.11: $\mathrm{BER}_{\mathrm{EVM}}$ and $\mathrm{BER}_{\text {theory }}$ vs. SNR.

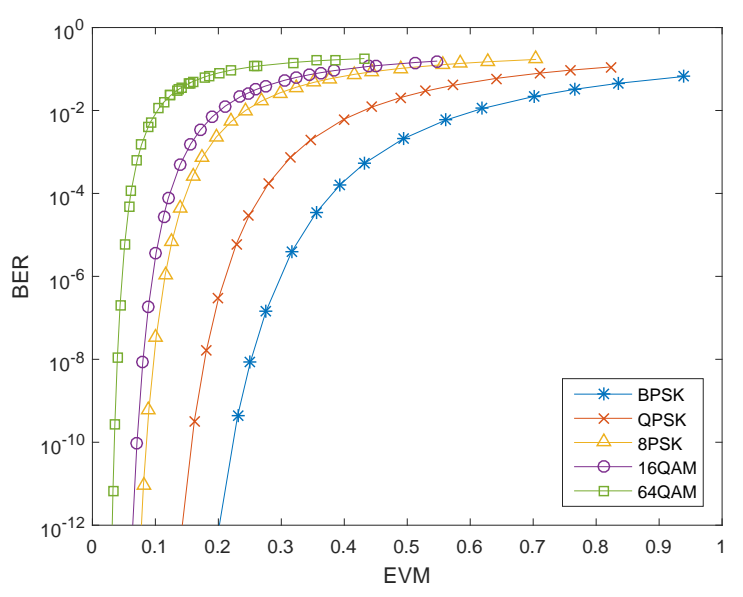

Figure 4.12: $\mathrm{BER}_{\mathrm{EVM}}$ vs. EVM. 
Table 4.6: EVM Thresholds for BER of $10^{-5}$

\begin{tabular}{lcc}
\hline & SNR $(\mathrm{dB})$ & EVM \\
\hline BPSK & 9.7 & 0.3282 \\
QPSK & 12.6 & 0.2333 \\
8PSK & 17.7 & 0.1284 \\
16QAM & 19.2 & 0.1060 \\
64QAM & 25.4 & 0.0525 \\
\hline
\end{tabular}

BER with $N=1000$ and the theoretical BER, denoted as black-dashed lines with the corresponding marker for each modulation scheme. In comparison to the requirements for bit error rate measurement shown in Section 4.2.1, EVM estimation requires only 1000 symbols, instead of 10,000,000 symbols at BPSK for BER measurement.

Similar to Section 4.2.2, switching thresholds for the modulation schemes must be defined in terms of EVM in order to determine when to adapt. Unlike with the SNR-BER relationship where BER decreases as SNR increases, BER increases as EVM increases, shown in Fig. 4.12. This relationship arises from a higher EVM corresponding to a lower SNR, as seen in the reciprocal relationship of (4.8).

The EVM thresholds are determined by the BER threshold, in the case of this work, $10^{-5}$, and then finding at which EVM value the BER decreases below the threshold for each modulation scheme. The thresholds in Table 4.6 are the exact point when the EVM-estimated BER decreases below the desired BER threshold.

To follow a similar approach as the SNR thresholds in Section 4.2.2, a hysteresis region needs to be established. The two scenarios that cause the receiver to switch with EVM are 
Table 4.7: Error Vector Magnitude Bounds for Receiver Switching Scenarios

\begin{tabular}{lrr}
\hline & SWITCH DOWN & SWITCH UP \\
\hline BPSK & $\mathrm{N} / \mathrm{A}$ & 0.2123 \\
QPSK & 0.2389 & 0.1173 \\
8PSK & 0.1293 & 0.0933 \\
16QAM & 0.1074 & 0.0468 \\
64QAM & 0.0531 & $\mathrm{~N} / \mathrm{A}$ \\
\hline
\end{tabular}

as follows:

1. Decreasing EVM: Receiver will switch from a lower to a higher modulation mode.

2. Increasing EVM: Receiver will switch from a higher to a lower modulation mode.

A decreasing EVM indicates that the received symbols are becoming closer to the reference symbols, and thus the error vector is shrinking. The lower error vector indicates that higher modulation schemes can be used for transmission while maintaining the BER threshold. Similarly, as EVM increases, the received symbols are moving further away from the reference symbols, lengthening the error vector. Thus, the EVM thresholds in Table 4.6 depict the absolute maximum EVM value for each modulation scheme while maintaining the BER threshold.

In order to adjust for disparity between estimations as well as adjust for slight variations in the incoming SNR, a hysteresis for the EVM thresholds is defined. To do so, the SNR threshold regions established in Table 4.4 define the EVM regions. To implement the hysteresis region, the "switch-down" value will be the absolute maximum EVM value supported by the modulation scheme while maintaining the BER threshold (Table 4.6), 


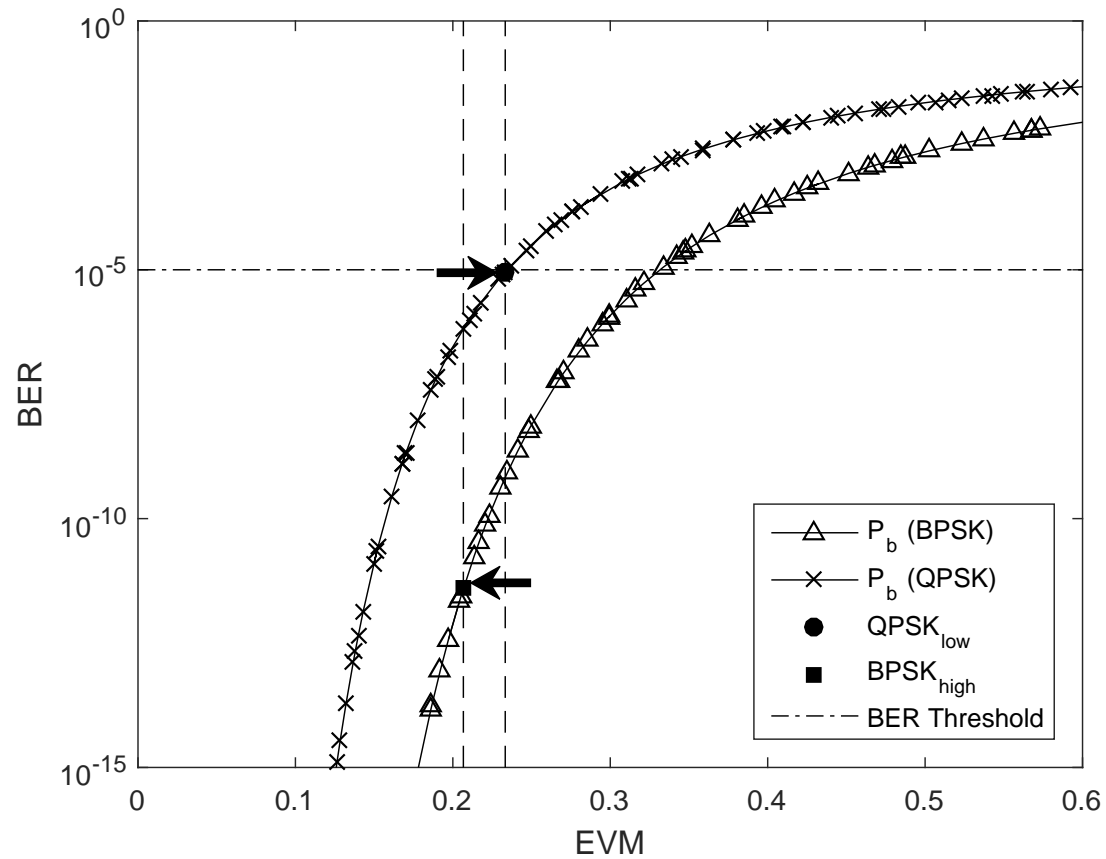

Figure 4.13: Demonstration of QPSK/BPSK EVM thresholds with hysteresis.

matching the minimum SNR from Table 4.4. However, for the "switch-up" level, the hysteresis region is implemented to account for any oscillation in the channel conditions. The EVM values follow the $1 \mathrm{~dB}$ conservative region as seen in Section 4.2.2. This guard region allows for the EVM to decrease well into the next mode region and ensures that, should the receiver switch, the higher modulation scheme could achieve the desired BER. The upper and lower boundaries for EVM are seen in Table 4.7.

Fig. 4.13 shows the EVM thresholds for switching between QPSK and BPSK. The left-pointing arrow shows the EVM value that must be measured by BPSK in order for QPSK to then be transmitted. The right-pointing arrow shows the maximum EVM value that QPSK can tolerate and maintain the required BER threshold. For example, the EVM 
maximum for QPSK is $0.2389^{1}$. To switch from BPSK to QPSK, the EVM needs to be 0.2076 or less. However, this 0.2076 value is the EVM measurement for the QPSK constellation. The BPSK EVM value that correlates to a QPSK EVM value of 0.2076 is 0.2123 . Therefore, when considering the EVM thresholds, in order to switch from a lower order to a higher order, the EVM threshold needs to measure the current modulation scheme EVM value and determine when its value has decreased to the minimum necessary for the higher order to begin transmission, as depicted in Fig. 4.13.

\subsection{Performance Analysis}

The algorithm developed focuses on the change in EVM measurement to determine when to adapt modulation schemes. As seen in Section 4.2.4, an estimation of EVM with $N=1000$ symbols returns a good approximation of the SNR and, consequently, BER. Thus, an average of 1000 EVM values is used to estimate the BER in this system. Before applying the developed switching method to the SCaN Testbed scenario, a comparison is made between the switching method if $E_{S} / N_{0}$ is known perfectly and based on the EVM-estimated SNR. Figures. 4.14 and 4.15 shows the comparison between the two link adaptation algorithms. Observe that the link adaptation using theoretical BER switches right at the absolute threshold of $E_{S} / N_{0}$, i.e. when the BER almost exceeds the specified threshold. Meanwhile, the EVM LA algorithm has a more conservative switching characteristic. As discussed, when the receiver switches to a higher modulation order, the channel quality must be sufficent to

\footnotetext{
${ }^{1}$ while maintaining a BER of $10^{-5}$
} 


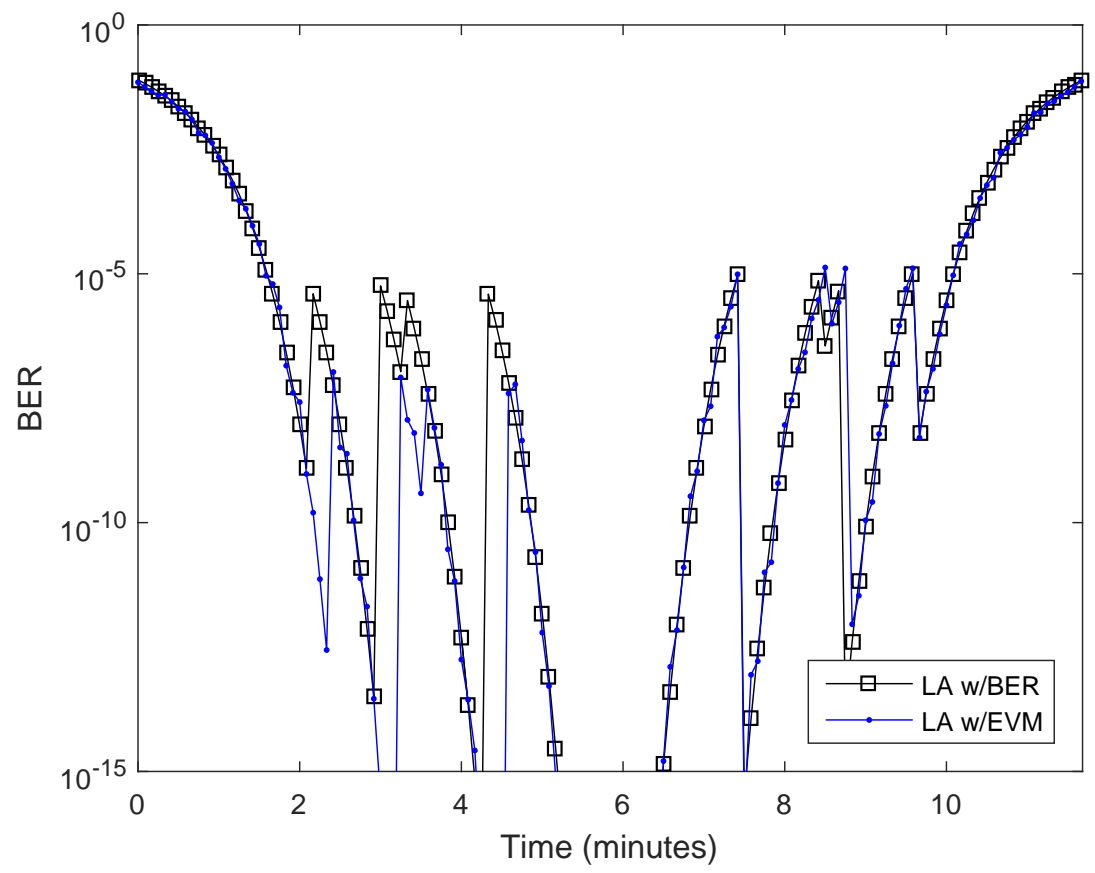

Figure 4.14: BER of LA Algorithms varying across time.

support the higher order while meeting the required BER threshold. Thus, the left side of Fig. 4.14 and Fig. 4.15 both show the EVM-estimated link algorithm switching after a delay in comparison to the BER-motivated algorithm. This delay allows for the EVM estimator to ensure that the estimator can accurately select the appropriate modulation scheme. However, when switching from high-to-low, the EVM-estimator more closely follows the theoretical algorithm as the "switch-down" thresholds represent the upper limit of the modulation scheme. So, waiting until the absolute final moment prior to switching down a modulation scheme allows for the maximization of the data throughput, while maintaining the BER threshold.

The switching delay evident in the EVM algorithm in Figs. 4.14 and 4.15, while ensuring 


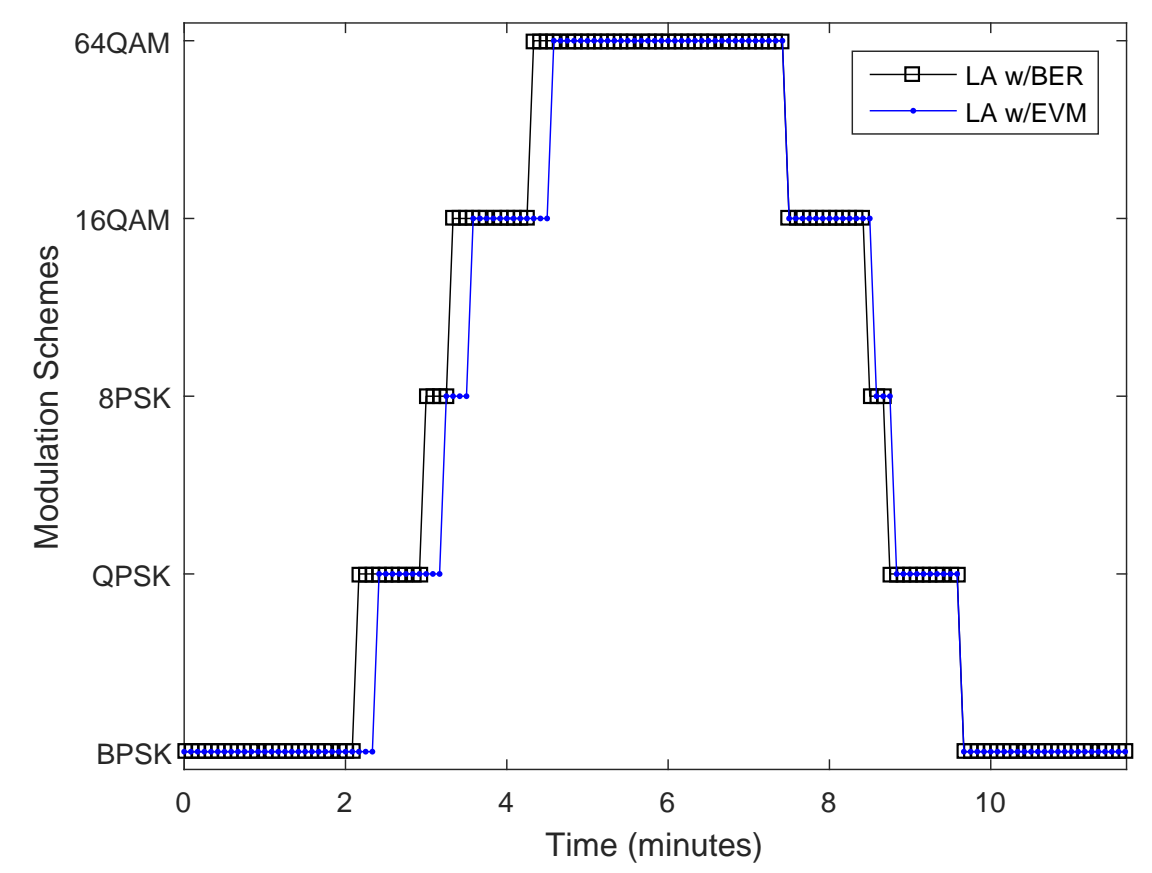

Figure 4.15: Modulation schemes of LA algorithms varying across time.

the operational BER requirement is met, limits the data throughput. As the algorithm does not switch at the first indication that the next modulation scheme can operate in the current channel conditions, it loses the throughput gain during those periods. Fig. 4.16 compares the BER-motivated, theoretical good throughput with the EVM-estimated algorithm. Both algorithms start out with the same throughput, but as soon as the BER algorithm switches to the new mode, it pulls ahead of the EVM algorithm for total transmitted bits. Additionally, a fixed modulation scenario is also depicted to compare the throughput against both the theoretical and EVM algorithm. The theoretical algorithm transmits $2.5 \%$ more data than the EVM-estimated algorithm; however, the EVM-estimated algorithm improves the data throughput of the fixed mode by $305 \%$. While the theoretical algorithm transmits more 


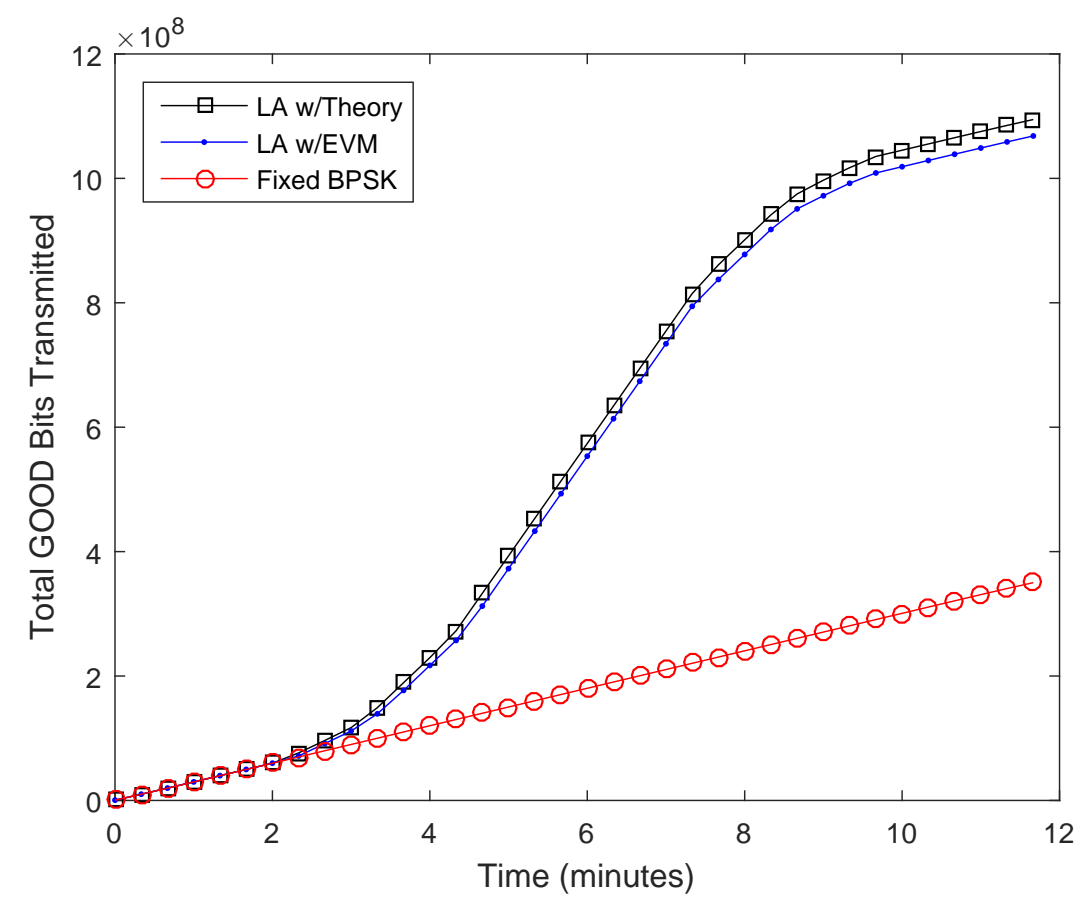

Figure 4.16: Total number of transmitted bits of each LA algorithm varying across time.

data than the EVM-estimated algorithm because of the hysteresis in switching modes, the EVM-estimated algorithm very clearly surpasses that of a fixed-modulation scheme, which is the current operation mode of many NASA communications. 


\section{Chapter 5}

\section{System Simulation}

This chapter combines the analysis described in the previous chapters to do a comprehensive system simulation of the algorithm using the propagation data. This simulation uses the channel characterization technique from Chapter 3 for the SCaN Testbed and incorporates the solar panel shadowing loss to test the switching method. The algorithm developed in Chapter 4 is applied to determine if the data transmission can be maintained and throughput improved through adverse conditions such as antenna shadowing by solar-panel.

The stages of the simulation are depicted in Fig. 5.1. The first step is to calculate the carrier-to-noise ratio from the pass data generated in STK, shown in Chapter 4. The carrier-to-noise ratio $(\mathrm{CNR})$ is shown to be equivalent to $E_{S} / N_{0}$ when root-raised cosine match filtering is assumed. Additionally, SNR is equivalent to $E_{S} / N_{0}$ when assuming perfect synchronization [65]. Thus SNR $=$ CNR. Through this relationship, the CNR is used to generate complex, Gaussian noise to add to the modulated, digital symbols. The relationship 


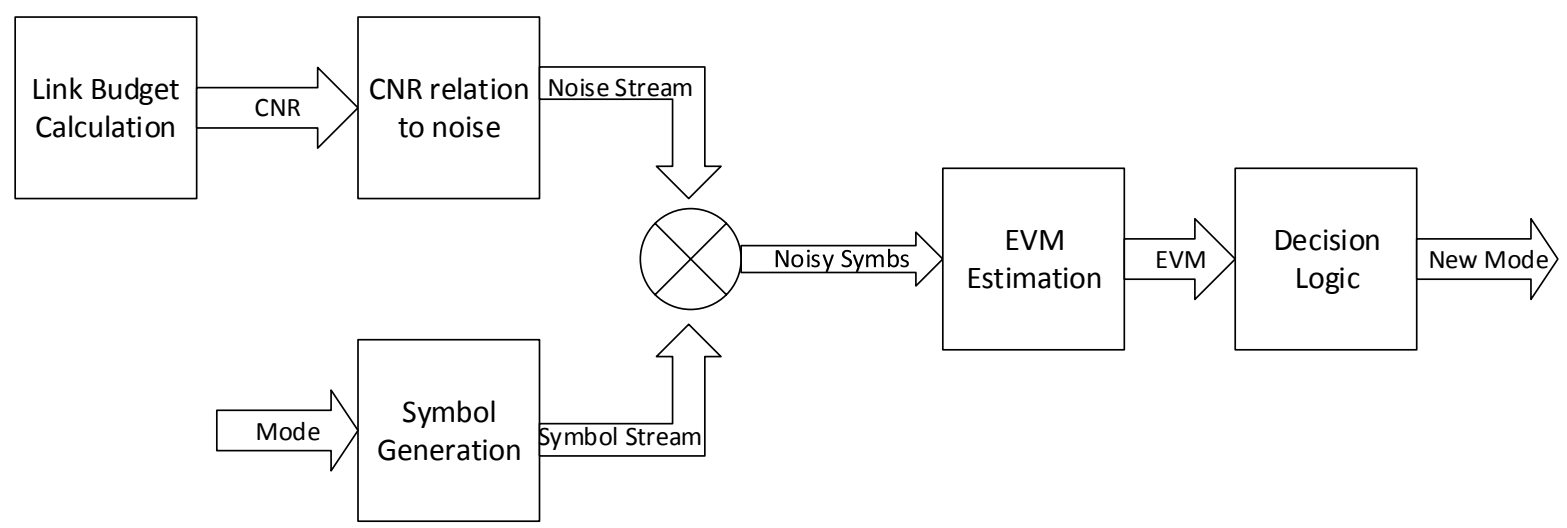

Figure 5.1: Flowgraph for the system simulation stages.

between SNR and the noise variance is, for bandpass signals [50]:

$$
\sigma_{n}^{2}=\frac{1}{\mathrm{SNR}}
$$

and thus, $\sigma_{n}^{2}=\frac{1}{\mathrm{CNR}}$.

This noise variance can be used, with the data calculated through the method in Chapter 3, to simulate the channel degradation experienced by the SCaN Testbed at each time step. A noisy symbol stream is generated by adding a generated noise stream to the true symbol stream. The true symbols are created with the current, modulation mode, and then the complex, gaussian noise is added to them. The noisy symbols are then passed into the EVM estimation. The STK-simulated $E_{S} / N_{0}$ data has a granularity of 1 second, so the EVM is assumed to be flat during the 1 second interval, and 1000 symbols are used to determine the estimate. In other words, the channel is assumed to not change drastically in 1 second. 
On-board the receiver, the granularity will be finer as the clocking frequency allows for measurements at the symbol rate.

The EVM measurement discussed in Section 4.2.4 is then performed on each symbol, using the symbol closest in distance as the ideal symbol. A moving average of EVM calculations over 1000 symbols is used to estimate the EVM value. When the average $\operatorname{EVM}_{[\mathrm{rms}]}$ shifts into a different modulation region, the receiver will switch modes. The initial receiver state is always BPSK, as it is the most robust to degraded channels out of the modulation scheme options used in this algorithm. Once an EVM estimation has been determined, the receiver will either maintain the status at BPSK or increase modulation rate/scheme, depending on the result of the estimate.

As improving the throughput of the uplink is one of the goals of this algorithm, Fig. 5.2 displays the average spectral efficiency across the first 20 passes. Spectral efficiency is calculated as

$$
\eta_{\text {spectral }}=\frac{R_{b}}{B}
$$

where $R_{b}$ is the net bit rate of the link and $B$ is the bandwidth of the signal. The bandwidth of the signal is $B=(1+\alpha) R_{b}$, where $\alpha$ is the roll-off of the root-raised cosine filter. The root-raised cosine filter, discussed more in Chapter 6, has an $\alpha$ of 0.75 .

In Fig. 5.2, each of the horizontal lines represents the spectral efficiency of a given modulation scheme, should that modulation scheme been used on a fixed-modulation receiver. Observe that the adaptive algorithm performs better than a purely BPSK fixed-modulation 


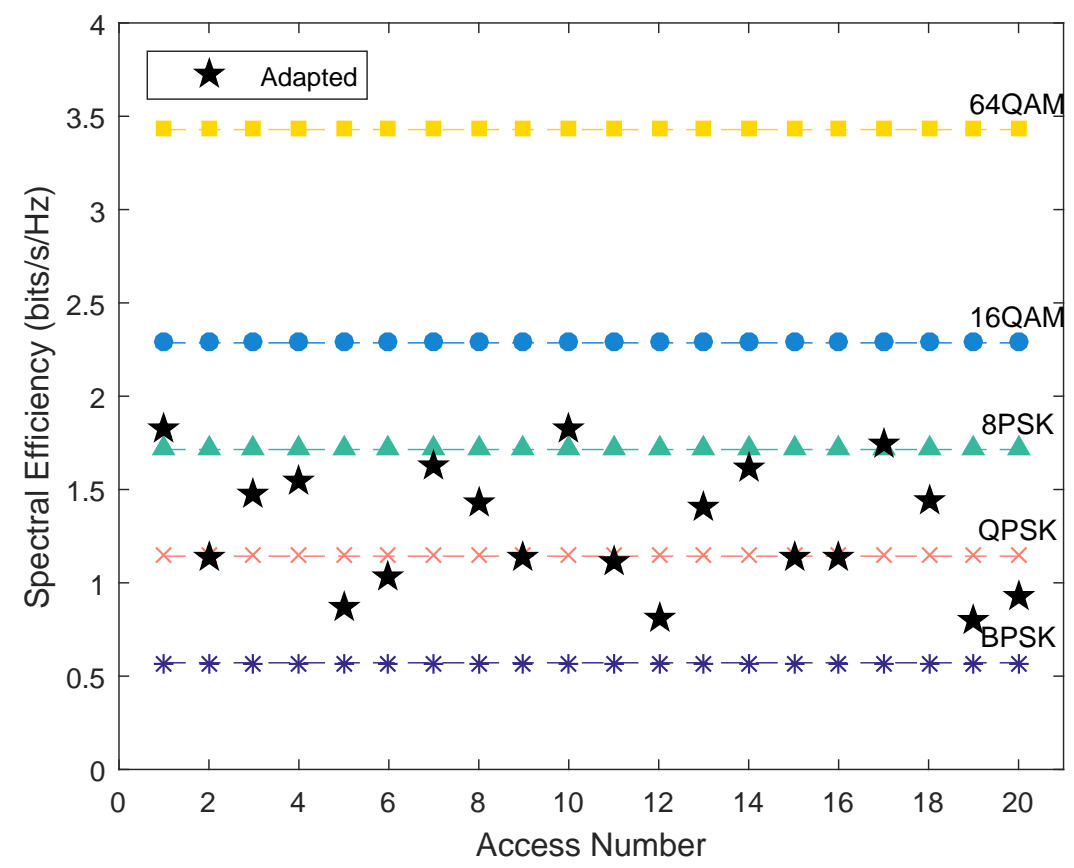

Figure 5.2: Spectral efficiency of LA algorithm compared to fixed-modulation.

receiver. As is expected, with no regard for meeting the BER threshold requirement, the higher order modulation schemes clearly have an improved spectral efficiency.

A more accurate comparison lies between the maximum fixed-modulation mode and the adaptive algorithm. To determine the fixed modulation scheme that would need to be transmitted in order to maintain the BER threshold, the minimum $E_{S} / N_{0}$ for each pass was calculated and compared to the thresholds determined in Section 4.2.2. The fixed-modulation good data throughput is compared to the adaptive modulation good throughput and the theoretical adaptive algorithm. The good throughput for the scenarios are shown following each figure. Observe that the adaptive algorithm at least matches, and often exceeds the throughput performance of the fixed modulator. 
A sampling of the 207 accesses analyzed is presented for comparison in the following figures. The following figures compare the EVM-estimated BER (the solid line) with the theoretical switching bit error rate. As can be seen in most plots, the theory-based LA method switches at a later time than the EVM-based algorithm. This is due to the hysteresis region and averaging of the EVM measurements. The EVM-based algorithm conservatively switches, ensuring the BER to be maintained below the $10^{-5}$ threshold before switching modes. Each scenario also has an accompanying data throughput plot, which shows the good data throughput across the entire pass.

Figure 5.3 shows a pass that has fairly symmetric switching stages, and switches across all five modulation schemes. The throughput matches the fixed modulation scheme of QPSK at the beginning of the pass, only to drastically start improving with the adaptive link, as seen in Fig. 5.4. Figure 5.5 shows a severe case of solar panel obstruction. Even though the receiver transmits BPSK, it is still unable to maintain the desired BER. However, prior to the solar panel shadowing degrading the channel, the receiver was able to receive QPSK at double the BPSK throughput, during that time. The throughput improvement is seen in Fig. 5.6. While the throughput improvement is not as large as the previous scenario, the adaptive receiver still doubled the throughput in comparison to the fixed receiver.

Figure 5.7 demonstrates a pass when the solar panel rotates into view of the antenna while operating on a high modulation scheme (16QAM). Note that the receiver switches quickly after determining that the EVM has degraded significantly. Most notably, the algorithm determines that it must switch to the lowest modulation order (BPSK) to minimize the 


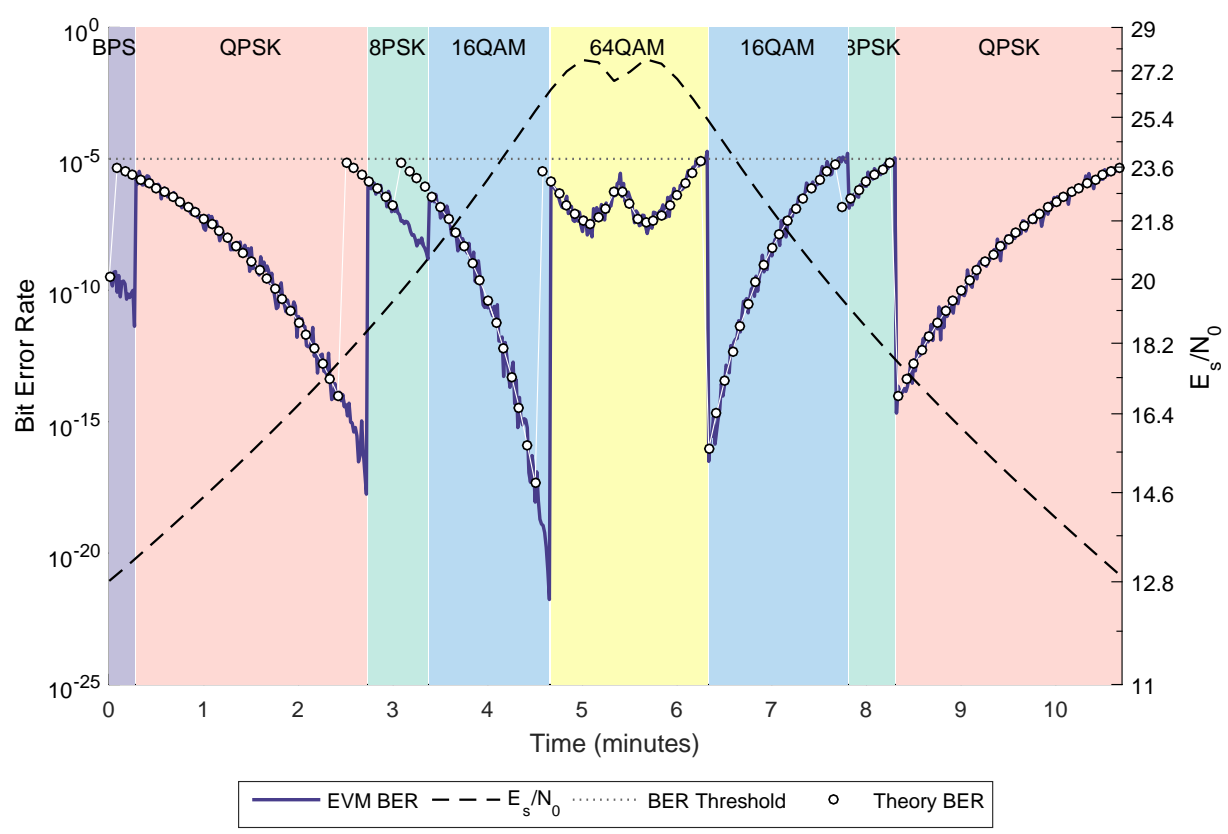

Figure 5.3: Switching scenario with all 5 modes.

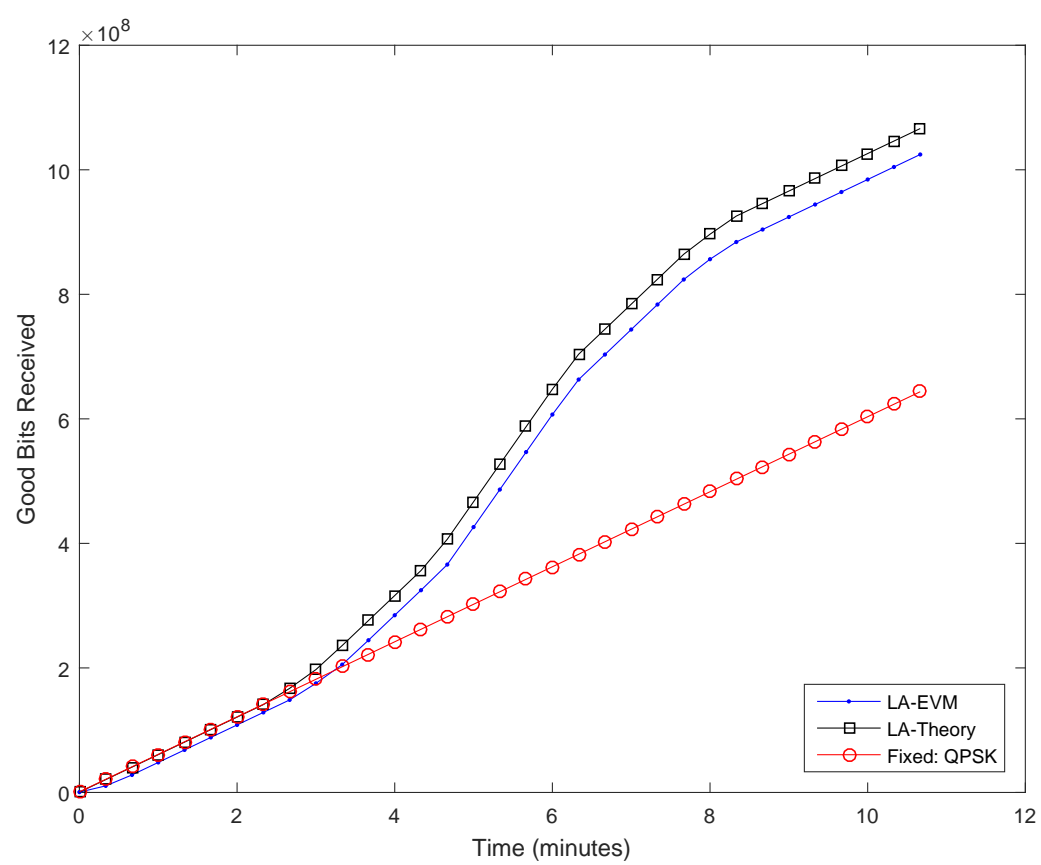

Figure 5.4: Data throughput for scenario in Fig. 5.3. 


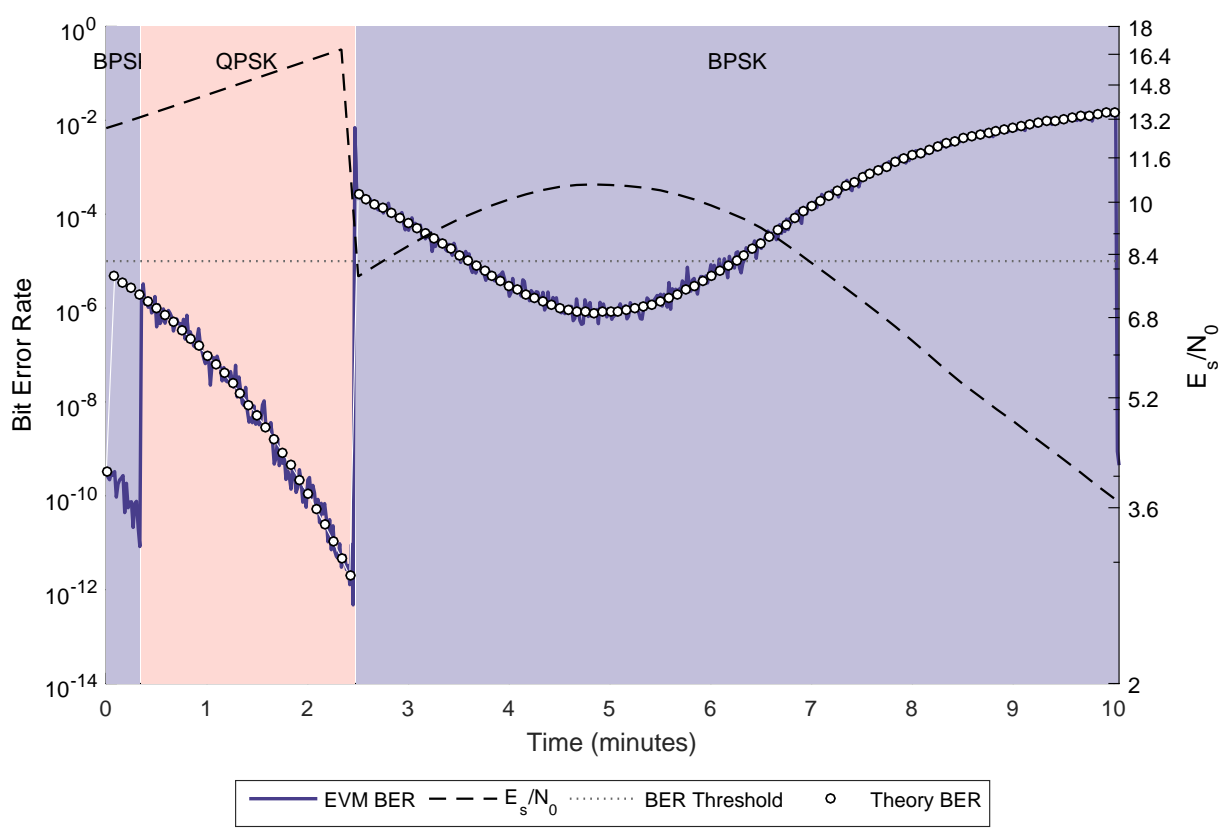

Figure 5.5: Access with severe shadowing.

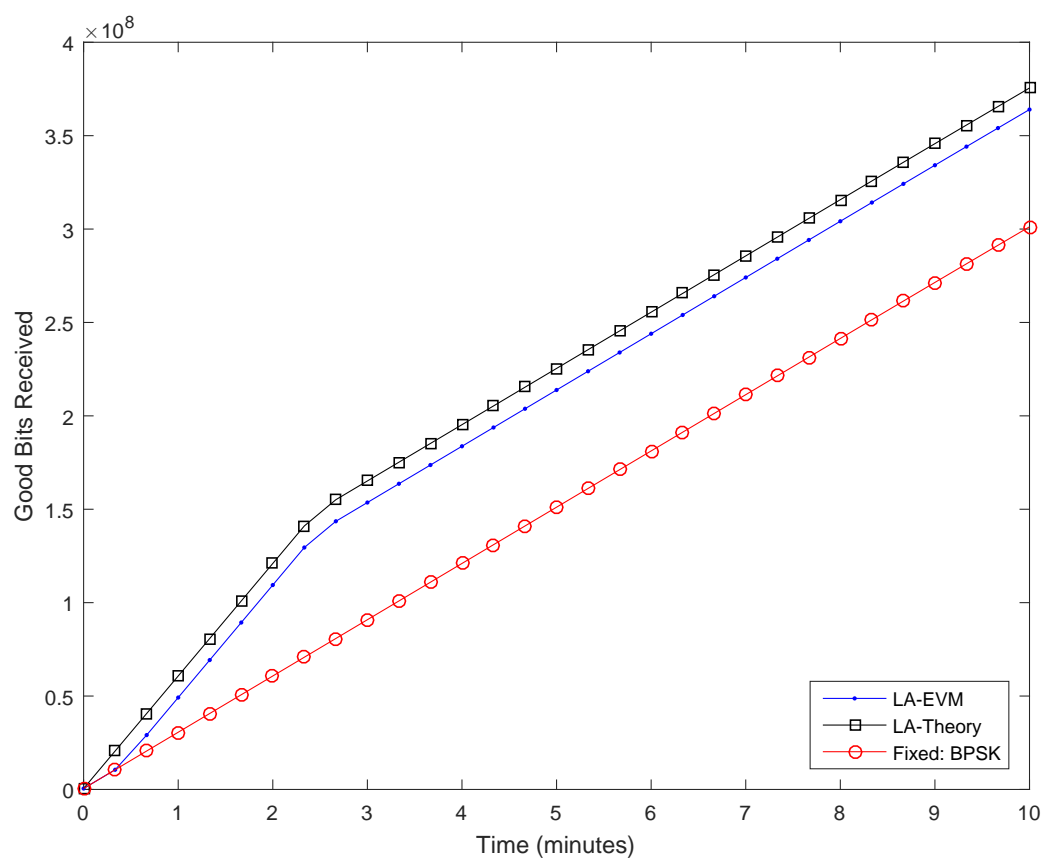

Figure 5.6: Data throughput for scenario in Fig. 5.5. 
BER; this differs from most other passes as the channel model is a gradual change between $E_{S} / N_{0}$ values which generally causes the modulation schemes to change linearly rather than jumping modes. The throughput lines in Fig. 5.8 also show when the receiver dropped modulation schemes, as there is a dip in the slope of the performance line. Even with the receiver returning to the lowest modulation order, the throughput of the overall pass is significantly improved from the fixed modulation scheme. Figure 5.9 shows an access that is shadowed at the beginning of the pass. The channel conditions are poor enough that even the most robust (BPSK) of modulation schemes were incapable of maintaining the preferred BER threshold. However, soon after the solar panels rotate out of view, the adaptive algorithm determines that 8-PSK can be received reliably. Thus, even though a pass may initially be degraded, the loss of throughput could be improved with the application of a link adaptation algorithm. The improved throughput in the latter half of the pass is shown in Fig. 5.10. The improvement in the adaptive receiver throughput shows that passes previously considered too poor for data transmission, now have the opportunity to leverage the higher link conditions with higher modulation modes. 


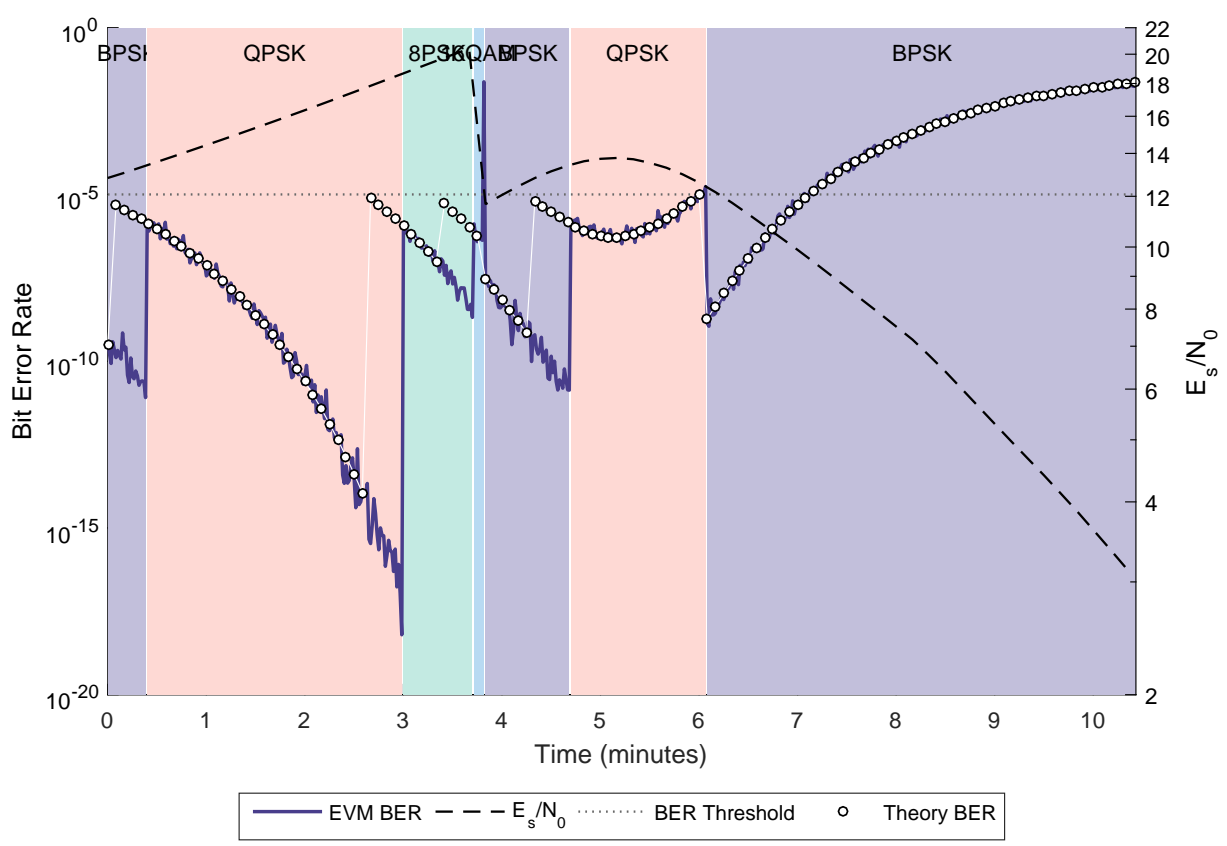

Figure 5.7: Access that shows jumping modes.

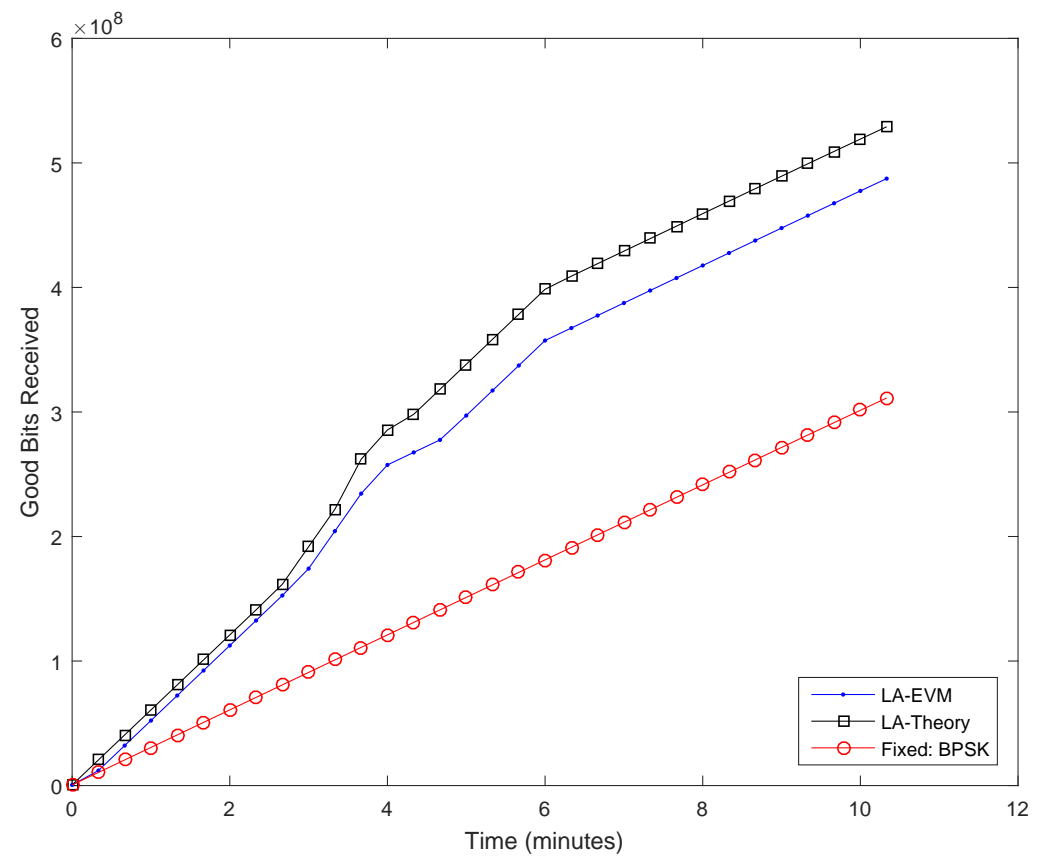

Figure 5.8: Data throughput for scenario in Fig. 5.7. 


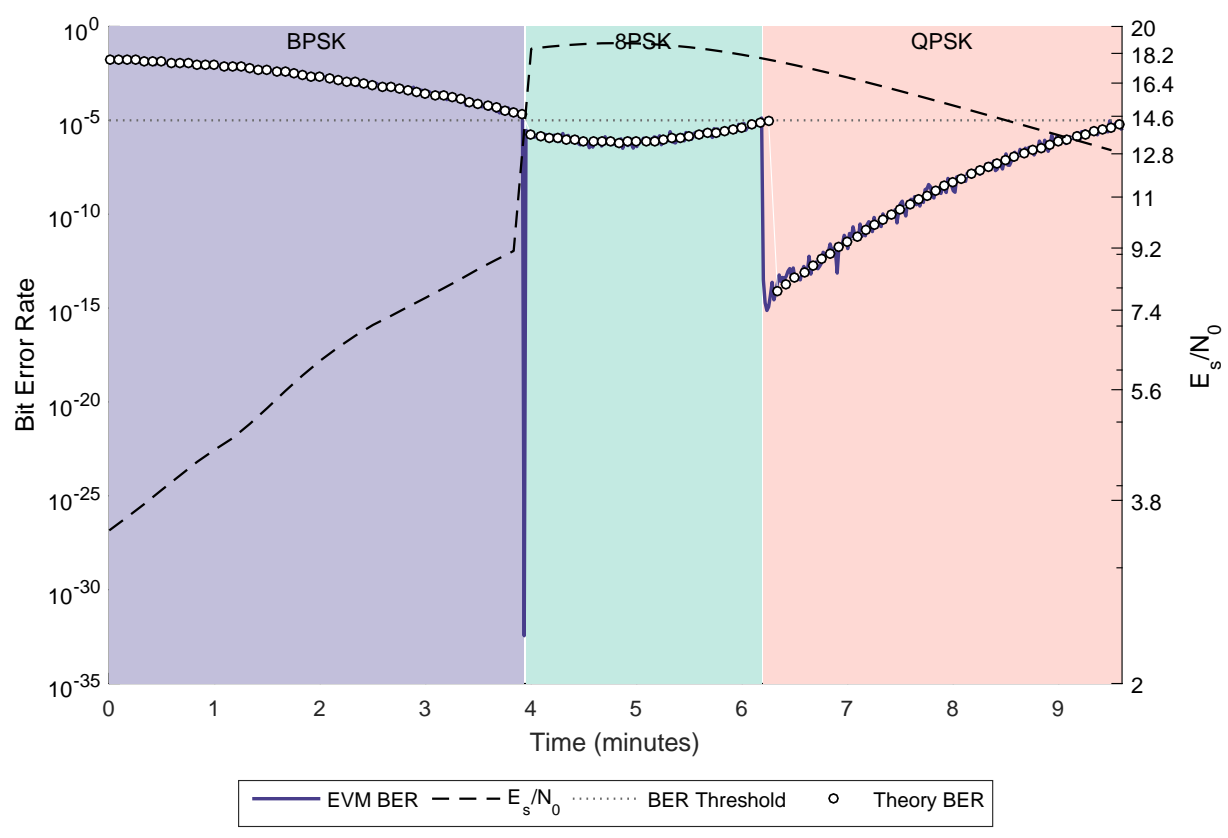

Figure 5.9: Shadowing and jump in mode.

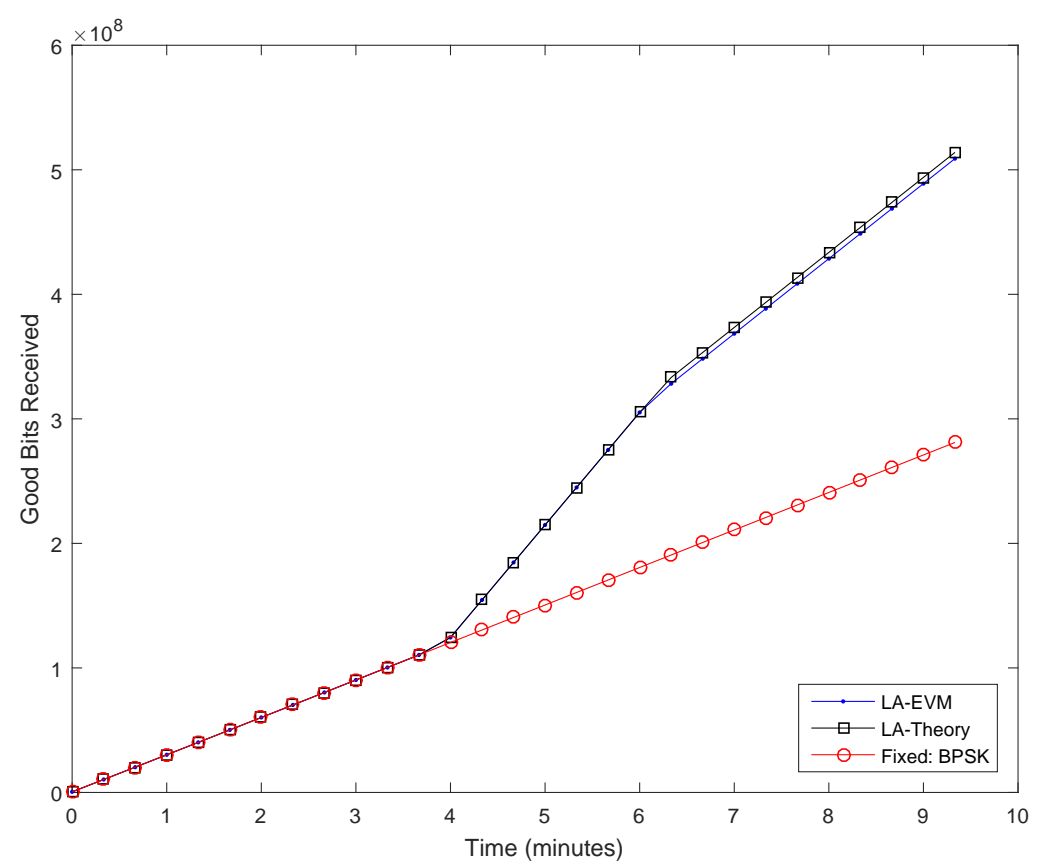

Figure 5.10: Data throughput for scenario in Fig. 5.9. 


\section{Chapter 6}

\section{Current and Future Work}

This chapter introduces the current development status of the on-flight experiment using the models and algorithm from the earlier chapters. The overview for both the transmitter and receiver are presented. Additionally, suggestions to expand the work described in the previous chapters are also discussed.

\subsection{Flight Experiment}

The algorithm developed and modeled in Chapters 4 and 5 is to be flown on the NASA SCaN Testbed JPL SDR. The two FPGAs installed on the radio are Virtex II 3M gates and the on-board general purpose processor (GPP) is a SPARC [17] GPP. 


\subsubsection{Xilinx System Generator}

In order to develop on Virtex IIs, version 10.1 of Xilinx ISE (Integrated Synthesis Environment) is used for most of the receiver and transmitter design as this version supports the hardware. This version of ISE is compatible with MATLAB 2007b. Thus, all development using MATLAB System Generator (SysGen) was performed in the 2007b release.

\subsubsection{Clock Rate Discussion}

In the following designs for the transmitter and receiver, the symbol rate and clock frequency are very different. As a design decision, the samples at the analog-to-digital converter (ADC) and digital-to-analog converter (DAC) are sent in at the clock frequency of the hardware: 49 MHz. The symbol rate for the incoming signal is $502 \mathrm{kSps}$. Therefore, in order to convert to the clock frequency of the DAC, an upsample value of 98 is included. Likewise, to return the symbol rate from the ADC frequency, a downsample of 98 is applied. This rate-change is evident in the following sections.

\subsubsection{FPGA Transmitter}

First, the development of the Experimental Path Downlink transmitter is addressed. The communications link incorporates a matched filter for pulse-shaping, discussed in more detail in Section 6.1.3.1. 


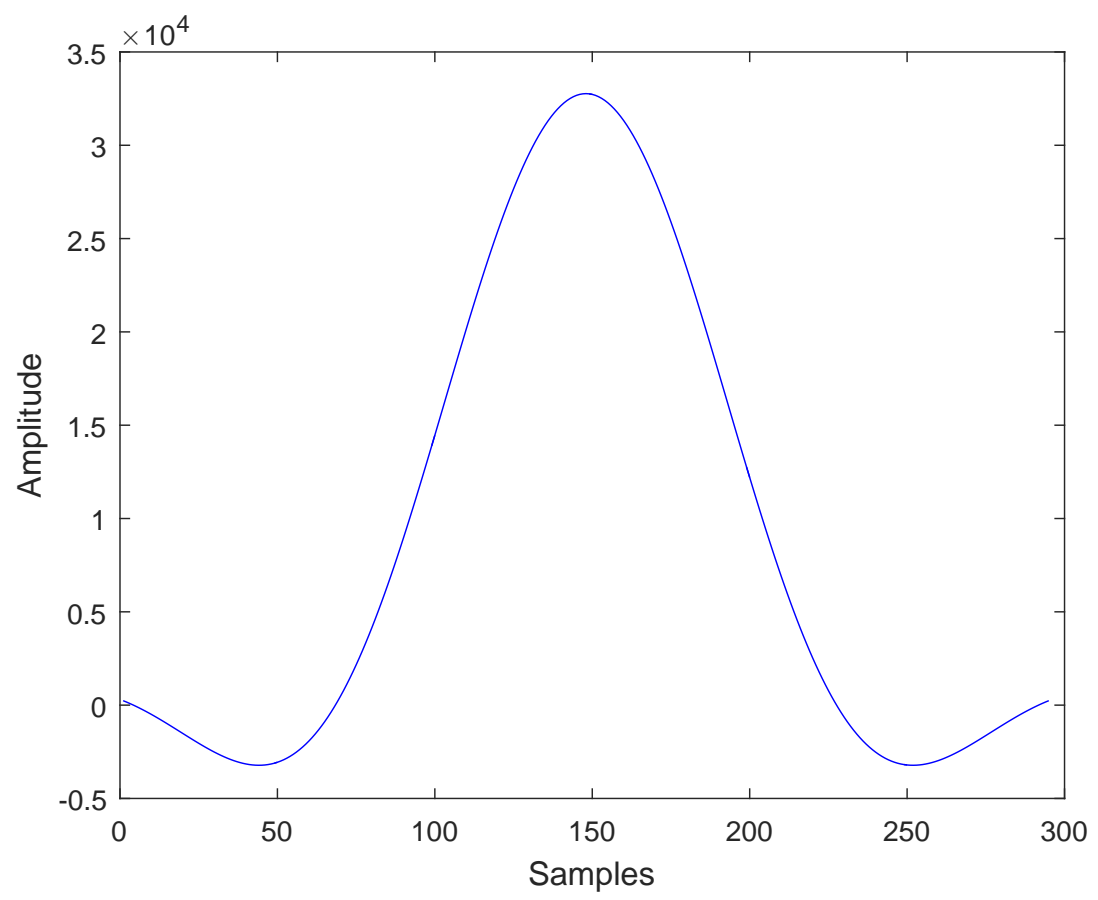

Figure 6.1: Time domain response of the root-raised cosine filter coefficients.

\subsubsection{Matched Filter}

The matched filter designed for this link is a root-raised cosine with a roll-off factor of 0.75 and a span of \pm 3 symbols. As discussed in Section 6.1.2, the filter must support a rate change value of 98 in order to deliver the samples to the DAC at the system clock rate and retrieve symbols from the $\mathrm{ADC}$ at the symbol rate. The filter coefficients were generated in MATLAB using the root-raised-cosine function. Fig. 6.1 displays the impulse response of the resulting coefficients. The filter is then imported into SysGen using the Xilinx CORE Generator to generate a Virtual Hard Disk (vhd) file. This file is then incorporated into full simulations of the transmitter. 


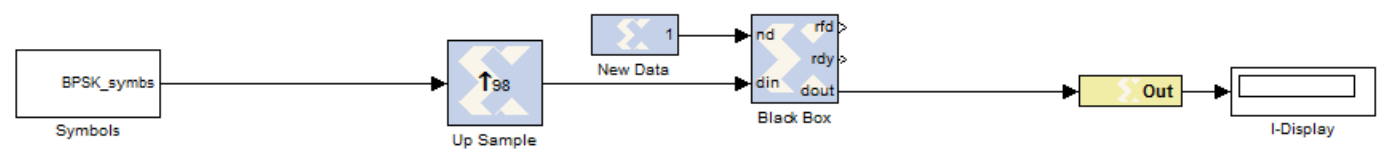

Figure 6.2: System generator transmitter design flowgraph.

As the downlink is a robust BPSK signal, the design of the transmitter is relatively simple. Fig. 6.2 shows the SysGen flowgraph of the transmitter. First, the BPSK symbols are randomly generated. On the final downlink design on the JPL SDR, the BPSK symbols will be formatted in the GPP, as the processor will determine the desired operating mode. In order to test the transmitter functionality, a random stream of data suffices.

Then, in order to deliver data to the DAC at its clock rate, the BPSK symbols are up-sampled by 98 . Following the upsampling, the data is sent through the matched filter ${ }^{1}$ designed in Section 6.1.3.1.

The SysGen model is then used to generate a Verilog file. This file is wrapped with the JPL SDR components (SPARC processor, DAC) to generate a .bit file. To test this .bit file, a stream of data is sent through and the output recorded using the FPGA simulator ModelSim.

This output is then sent through a matched filter receiver and down-sampled in order to

\footnotetext{
${ }^{1}$ The matched filter is represented as a "Black Box" in Xilinx SysGen because the filter was designed outside SysGen and imported as a .vhd file.
} 


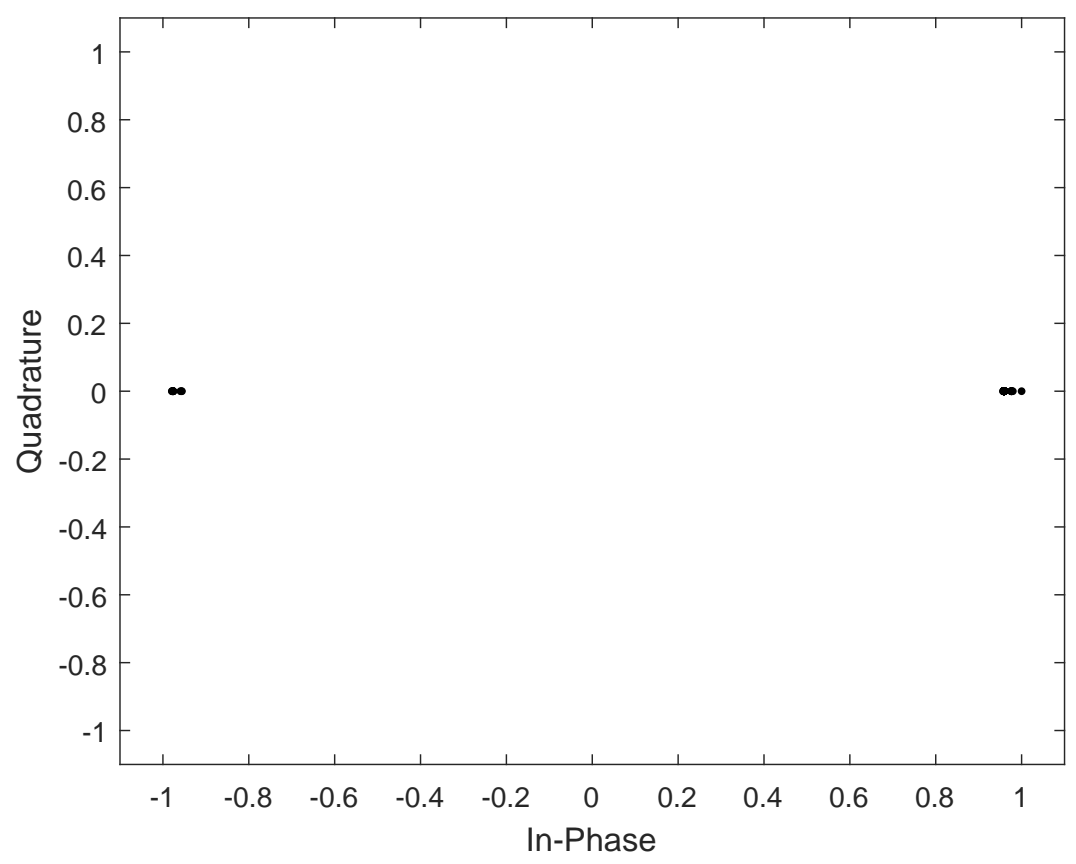

Figure 6.3: "Received" symbols from designed transmitter after matched filtering.

recover the original transmitted bit stream. Fig. 6.3 shows the received symbols after the matched filter. The pin-points are very close together, but not perfectly aligned as to be expected due to inter-symbol interference (ISI) from the matched filter filter. The symbols are then demodulated so that the received symbols can be compared with the transmitted bits. After adjusting for the delay introduced by the filter, it is evident that the bits transmitted are accurately reconstructed in this test, as seen in Fig. 6.4.

This test shows that the transmitter is ready for the next stage of development: prototype testing. GRC has a JPL SDR prototype available for testing, so the design will be verified using this tool. 


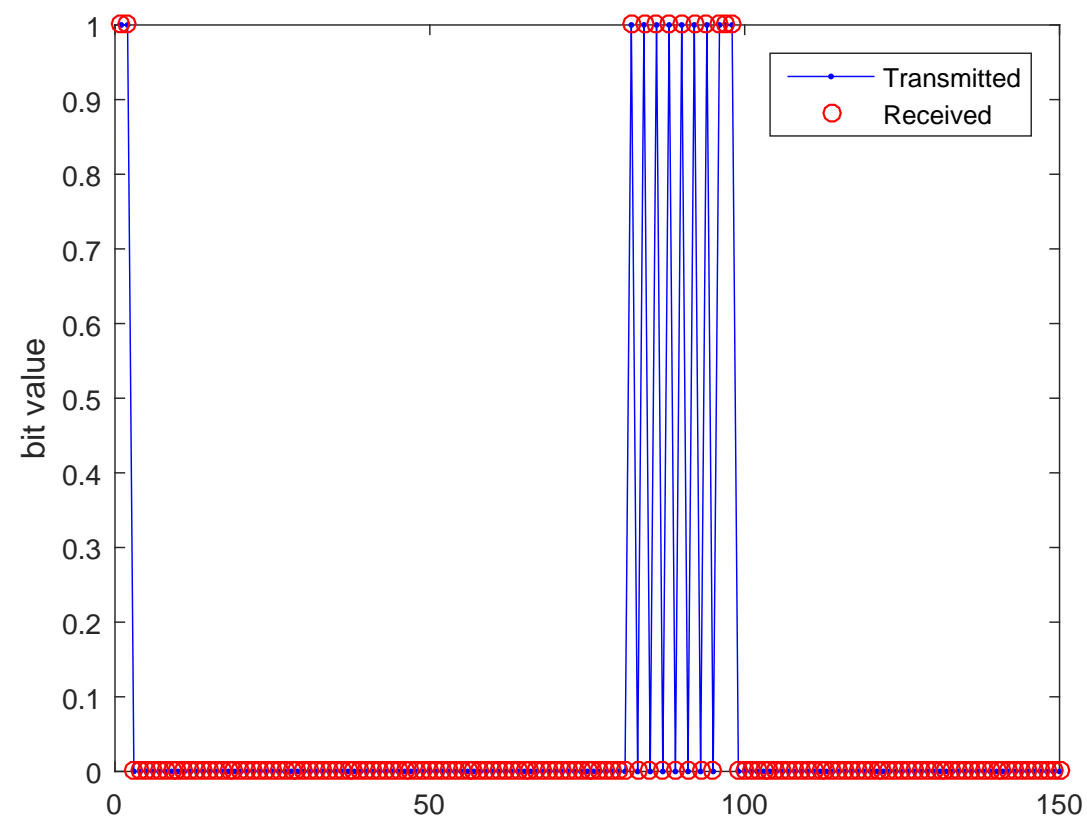

Figure 6.4: Comparison of transmitted and received bit values.

\subsubsection{FPGA Receiver}

The preliminary receiver design is shown in Fig. 6.5. As the receiver is still in simulation stage, the received values are generated using MATLAB, the first box in the flowgraph. The received values are passed in and the matched filter from Section 6.1.3.1 is applied. Then, the data is down-sampled by 98 to recover the symbol rate. The receiver then works to achieve perfect synchronization through timing error estimation. Finally, the receiver will make hard decisions on the received symbols. The hard decision greatly reduces the bit representation necessary for the output.

Additionally, during the hard decision process, the error vector magnitude calculation is 


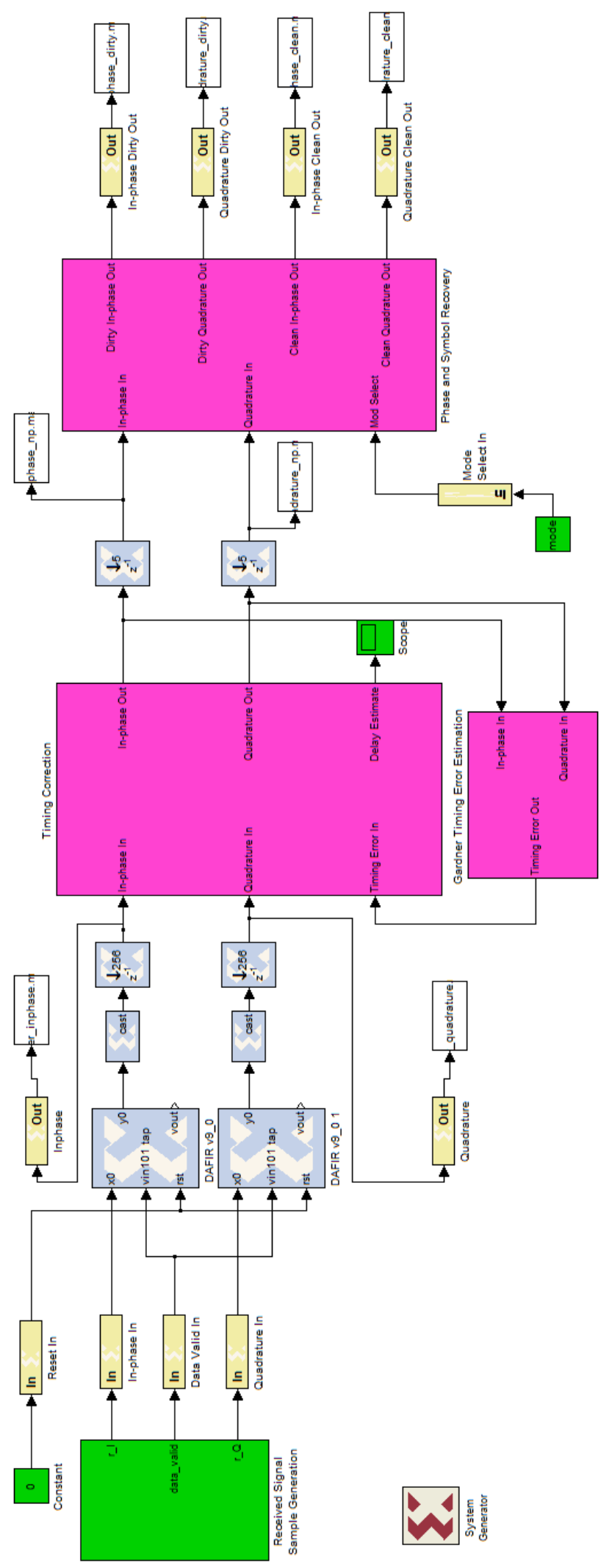

Figure 6.5: System Generator receiver design flowgraph. 
performed. The EVM will be implemented as a moving average of 1024 samples, as 1024 greatly simplifies binary division. After the initial system start-up, the moving average provides a granularity at the symbol period of $2 \mu \mathrm{s}$. This granularity is an improvement upon the STK simulation in Chapter 5. This EVM moving average value is used in the GPP for the decision logic. Thus, the receiver passes three values to the processor: the in-phase value of the received symbol, the quadrature value of the received symbol, and the error vector magnitude.

Currently, the receiver is still in the SysGen simulation stage, with a few final steps yet to be performed. First, the matched filter .vhd must be incorporated into the system, as the current filter is a generic root-raised cosine. Additionally, the EVM calculation and moving average must be implemented. Finally, the direct-digital synthesizer is introducing an error in the received soft symbols. As seen in Fig. 6.6, the received constellation for QPSK shows that most of the symbols are clustered in an inner constellation, but some symbols are outside the constellation diagram. Following the completion of the receiver design in SysGen, the module will be incorporated into the JPL SDR wrappers for the processor and ADC, similar to the method used for the transmitter.

\subsubsection{GPP Algorithm}

From the FPGA receiver design, the EVM moving average is transferred to the SPARC general purpose processor (GPP). Prior to installation on the JPL SDR, the EVM thresholds will be determined so they will operate as a look-up table. The comparison to the thresholds 


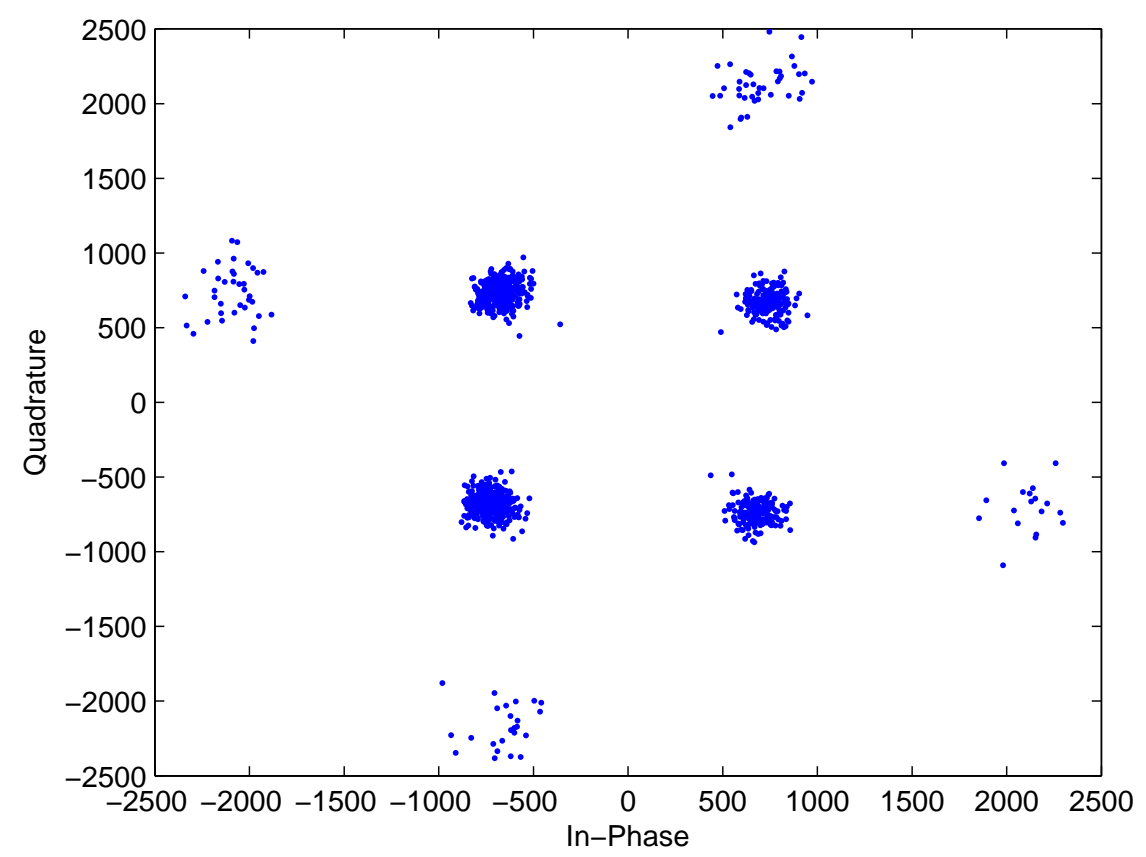

Figure 6.6: DDS-introduced error on received symbols after matched-filtering.

will be performed within the processor. If the logic indicates that a switch is necessary, the new mode will be included in the downlink packet to alert the ground station transmitter.

While the EVM thresholds for a BER of $10^{-5}$ are already defined, the $\mathrm{C}$ code must be written and simulated in the RTEMS (Real-Time Executive for Multiprocessor Systems) operating system. The RTEMS development environment used by NASA is installed in a virtual machine for testing and debugging of the logic. 


\subsection{Future Work}

The current decision metric used in the algorithm focuses on thresholds of EVM and a moving average. As the shadowing generally introduces a large drop in the received $E_{S} / N_{0}$, the resulting signal strength curve is not continuous. An alternative to comparing against thresholds would be to apply the second derivative to the measured EVM data (and estimated SNR). Should the second derivative display a discontinuity-an indication of severe shadowing-the receiver could then immediately switch.

One link adaptation approach applied the Maximum Likelihood criteria to determine the hysteresis window [29]. This approach would optimize the threshold windows as the method in Chapter 4 is a conservative design. Other LA approaches that would further this work are adaptive coding and power adaptation. Adaptive coding would provide coding gain, and potentially allow the modulation schemes to operate for a longer range of SNR values. Power adaptation would also allow higher modulation schemes to be sent under worse link conditions.

Additionally, rather than using a robust BPSK downlink, the Experimental Path Downlink could also be capable of adaptive modulation. Then, both the uplink and downlink could implement modulation classification in the link adaptation. There are some concerns with modulation classification, such as synchronization and number of samples available for estimations. However, ultimately, both links dynamically switching could expand the application base of this technique. 


\section{Chapter 7}

\section{Conclusions}

This work characterized the propagation effects experienced by the NASA SCaN Testbed, especially the effect antenna obstruction through solar-panel shadowing can have on the received signal power. Then, a proposed link adaptation algorithm leveraging error vector magnitude was presented and modeled. It was seen that the LA algorithm improved data throughput by using an adaptive modulation receiver.

The analysis of the simulation results has shown that an adaptive modulation receiver will perform better than fixed-modulation in improving data throughput while minimizing the bit error rate on-board NASA's SCaN Testbed. This was done by first analyzing the propagation effects experienced by the NASA's SCaN Testbed on-board the ISS. The challenge of solar panel shadowing is introduced as an effect that should be minimized. Next, the full propagation analysis was used to develop an adaptive modulation switching algorithm that monitors the estimated bit error rate of the system and adapts accordingly. 
The estimation of BER is done by performing an error vector magnitude (EVM) calculation on the received symbols. It is shown that for a larger number of symbols used in the estimation, the BER estimation becomes more accurate. Thresholds of EVM for each of the modulation schemes are defined for when switching should occur.

The simulations of this algorithm using the propagation environment of the Testbed demonstrate that a properly implemented adaptive modulation receiver can provide a better trade-off between BER and throughput than a fixed modulation receiver. This algorithm improves upon NASA's traditional communication approach by maintaining data transmission during times of decreased link quality, albeit at a lower throughput.

Given the successful results of this work, an on-orbit test on-board the ISS is planned to test the performance of the LA algorithm in-flight. Adaptive data rate links are the first step towards a more intelligent system. Coupling cognition with these adaptive waveforms will provide even more efficiency for the communication links. Applications will monitor and learn link behavior, predict propagation losses, recognize and avoid interference, and cooperatively use the spectrum. Capabilities such as these would increase the amount of data returned from NASA missions in the future. 


\section{Appendix A}

\subsection{TLE and SGP4}

In the instantiation of the ISS orbit, two-line element (TLE) data and the Simplified General Perturbations (SGP)-4 model are used to provide an accurate propagation routine. TLEs provide the position of an Earth-orbiting object for a given point in time. From this data, a prediction routine, such as SGP4, can be used to predict the position of the satellite at any point in time.

\subsection{Propagation Modeling Complete Table of Values}

The values used in the link budget analysis of Chapter 3 can be seen in Table A.1. All values were discussed when used in calculations, but the table allows for an overall summary. 
Table A.1: Summary of Propagation Analysis Characteristics and Calculations

\begin{tabular}{llrl}
\hline \multicolumn{3}{l}{ Path Loss Characteristics } & \\
\hline & & Minimum & Maximum \\
$d$ & Range & $396.88 \mathrm{~km}$ & $2328.67 \mathrm{~km}$ \\
$\lambda$ & Wavelength & $0.14 \mathrm{~m}$ & $0.14 \mathrm{~m}$ \\
$n$ & Free-space Loss Exponent & 2 & 2 \\
$L_{P}$ & Path Loss & $150.7 \mathrm{~dB}$ & $166.1 \mathrm{~dB}$
\end{tabular}

\begin{tabular}{llr}
\hline \multicolumn{2}{c}{ GRC Ground Station Specifications } \\
\hline$f$ & S-band Frequency & $2.07 \mathrm{GHz}$ \\
$P_{T X}$ & Transmitter Power & $10 \mathrm{~W}$ \\
$R_{S}$ & Symbol Rate & $2.0 \mathrm{MSps}$ \\
$d$ & Antenna Diameter & $2.4 \mathrm{~m}$ \\
$\eta$ & Efficiency & $70 \%$ \\
\hline
\end{tabular}

\begin{tabular}{llr}
\hline \multicolumn{2}{l}{ Transmitter Antenna Gain } \\
\hline$\eta$ & Efficiency & 0.70 \\
$d$ & Antenna Diameter & $2.4 \mathrm{~m}$ \\
$\lambda$ & Wavelength & $0.14 \mathrm{~m}$ \\
$G_{T X}$ & Transmitter Antenna Gain & $32.5 \mathrm{dBi}$
\end{tabular}

\begin{tabular}{llr}
\hline \multicolumn{2}{l}{ Effective } & Isotropically Radiated Power (EIRP) \\
\hline$P_{T X}$ & Transmitter Power & $10 \mathrm{dBW}$ \\
$G_{T X}$ & Transmitter Antenna Gain & $32.5 \mathrm{dBi}$ \\
$L_{T X}$ & Transmitter Losses & $2 \mathrm{~dB}$ \\
EIRP $_{T X}$ & EIRP & $40.5 \mathrm{dBW}$
\end{tabular}

\begin{tabular}{llr}
\hline \multicolumn{2}{c}{ Received Power Worst-Case } \\
\hline EIRP $_{T X}$ & EIRP & $40.5 \mathrm{dBW}$ \\
$L_{P}$ & Path Loss & $166.1 \mathrm{~dB}$ \\
$L_{\text {atmo }}$ & Atmospheric Loss & $1 \mathrm{~dB}$ \\
$L_{\text {iono }}$ & Ionospheric Loss & $1 \mathrm{~dB}$ \\
$G_{R X}$ & Receiver Antenna Gain & $-6.5 \mathrm{dBi}$ \\
$P_{R X}$ & Received Power & $-134.1 \mathrm{dBW}$ \\
& & \\
\hline System & Temperature \\
\hline$T_{r e f}$ & Reference Temperature \\
NF & Noise Figure & $290 \mathrm{~K}$ \\
$T_{R X}$ & Receiver Temperature & $5 \mathrm{~dB}$ \\
$T_{a n t}$ & Antenna Temperature (estimated) & $200 \mathrm{~K}$ \\
$T_{\text {sys }}$ & System Temperature & $632.5 \mathrm{~K}$ \\
\multicolumn{2}{c}{ Noise Power } \\
\hline$k$ & Boltzmann's Constant \\
$T_{\text {sys }}$ & System Temperature \\
$B_{n}$ & Noise Bandwidth \\
& & $632.5 \mathrm{~K}$ \\
\hline
\end{tabular}




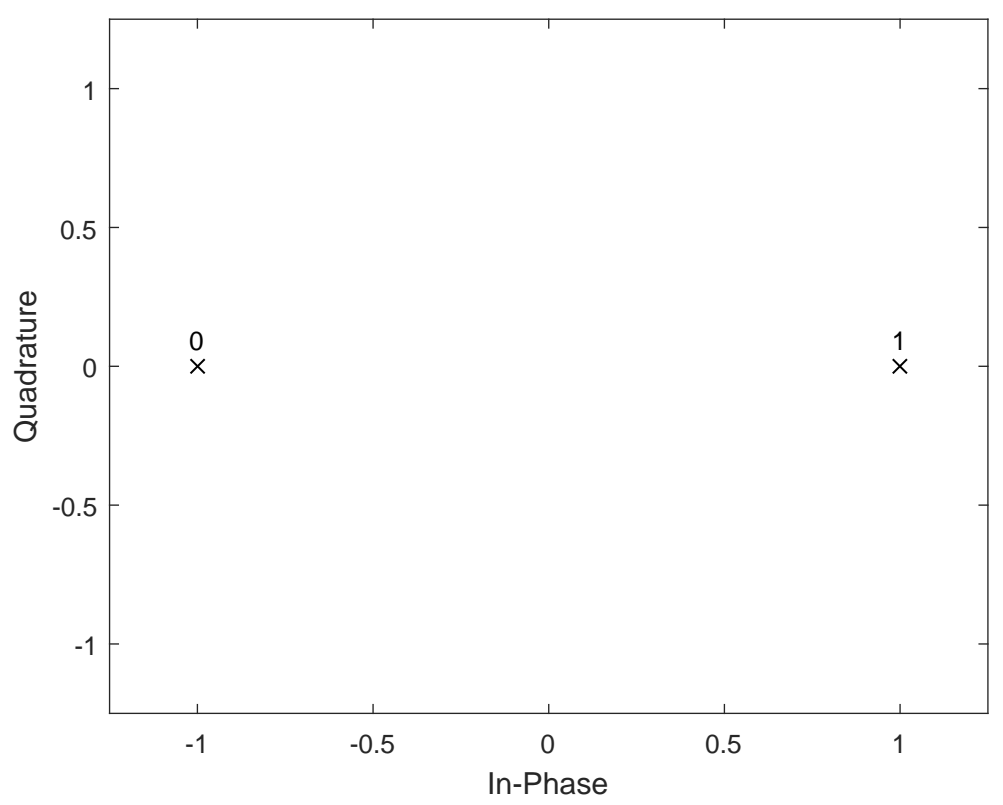

Figure A.1: BPSK Gray Code Arrangement.

\subsection{Gray Coded Modulation Schemes}

Each of the five modulation schemes used within this work are mapped using gray coding. Gray coding specifies that the bit representation of neighboring symbols differ by only one bit. The one bit difference greatly reduces the probability of two bits being in error simultaneously. Figures A.1-A.5 show the gray coding arrangement for BPSK, QPSK, 8PSK, 16QAM, and 64QAM, respectively. 


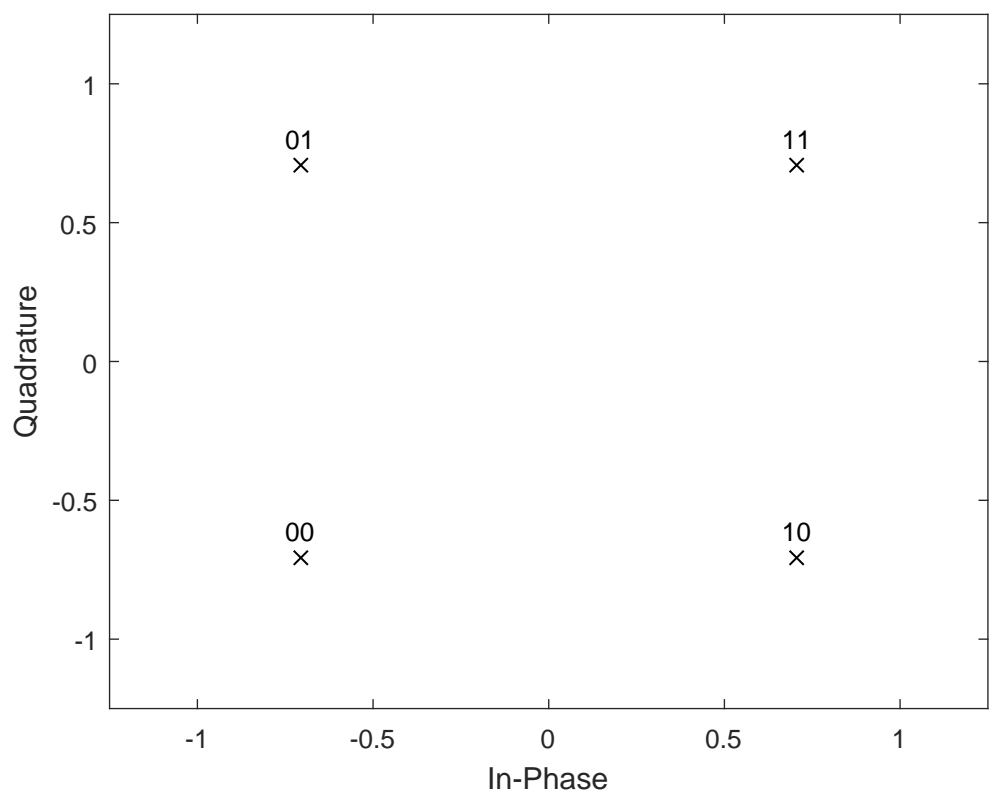

Figure A.2: QPSK Gray Code Arrangement.

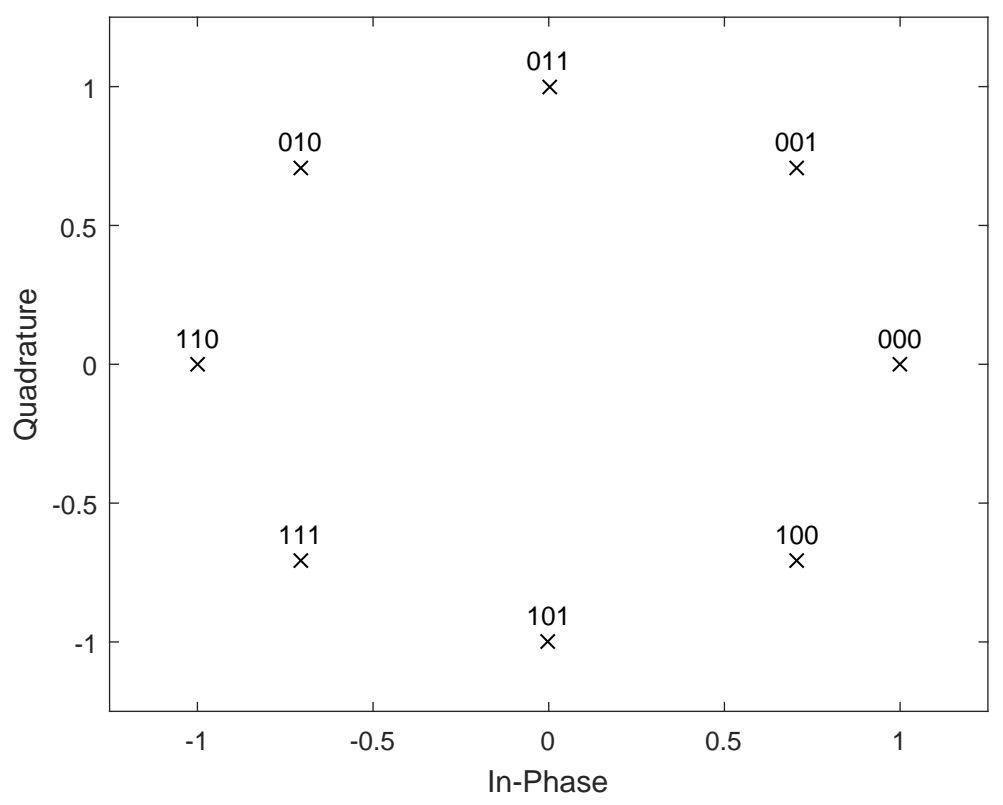

Figure A.3: 8PSK Gray Code Arrangement. 


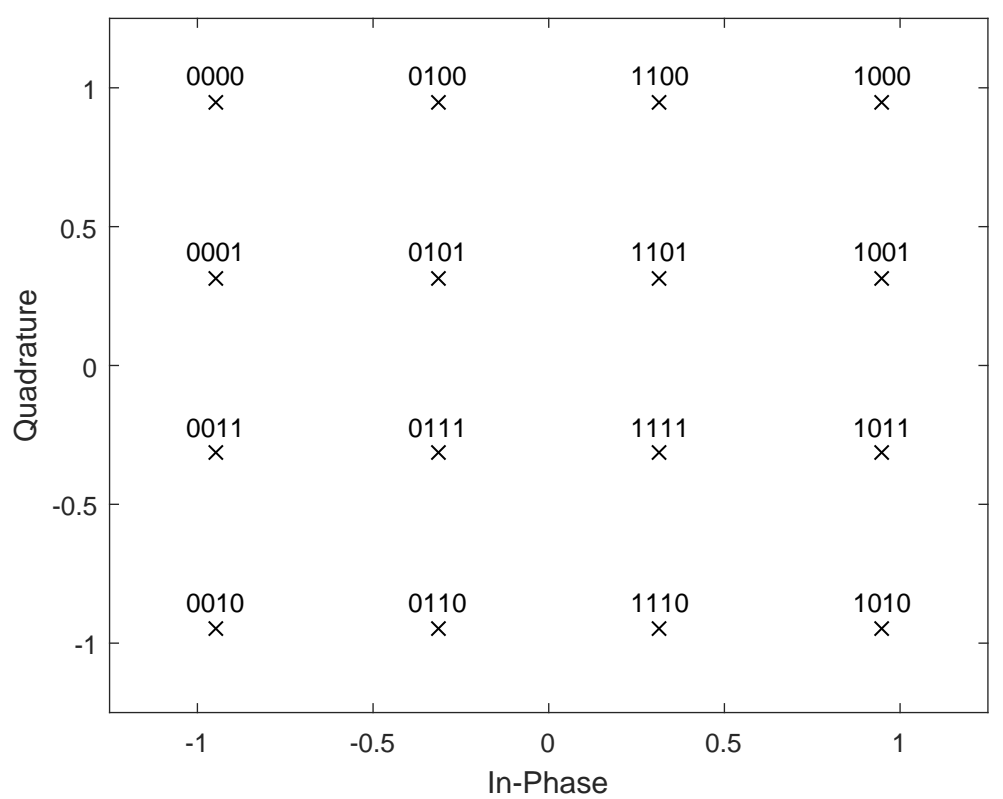

Figure A.4: 16QAM Gray Code Arrangement.

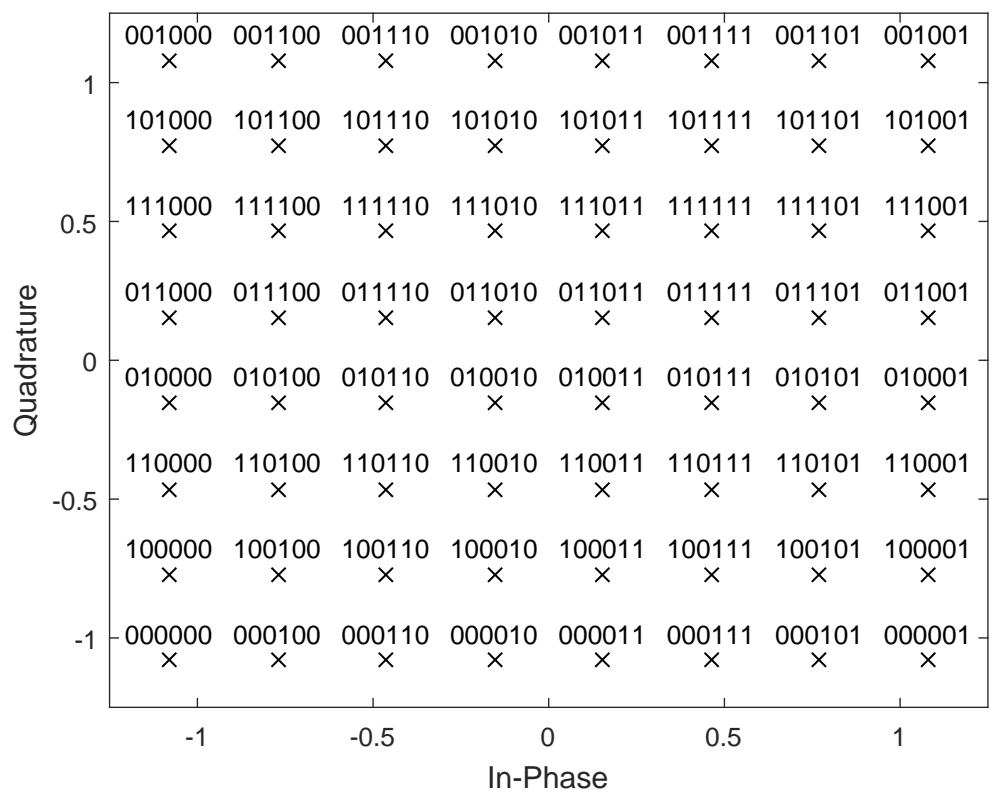

Figure A.5: 64QAM Gray Code Arrangement. 


\section{Bibliography}

[1] A. Arun and T. Sreeja, "An effective downlink budget for 2.24 ghz s-band leo satellites," in Information 8 Communication Technologies (ICT), 2013 IEEE Conference on. IEEE, 2013, pp. 342-345.

[2] J. Deloo, "Analysis of the rendezvous phase of e.deorbit," Master's Thesis, TU Delft, Delft University of Technology, 12015.

[3] K. Baum, T. Kostas, P. Sartori, and B. Classon, "Performance characteristics of cellular systems with different link adaptation strategies," Vehicular Technology, IEEE Transactions on, vol. 52, no. 6, pp. 1497-1507, Nov 2003.

[4] E. Armanious, D. Falconer, and H. Yanikomeroglu, "Adaptive modulation, adaptive coding, and power control for fixed cellular broadband wireless systems: some new insights," in Wireless Communications and Networking, 2003. WCNC 2003. 2003 IEEE, vol. 1, March 2003, pp. 238-242 vol.1.

[5] N. Mehta and A. Goldsmith, "Performance analysis of link adaptation in wireless data networks," in Global Telecommunications Conference, 2000. GLOBECOM '00. IEEE, vol. 3, 2000, pp. 1422-1426 vol.3.

[6] S. Ci and H. Sharif, "An link adaptation scheme for improving throughput in the ieee 802.11 wireless lan," in Local Computer Networks, 2002. Proceedings. LCN 2002. 27th Annual IEEE Conference on, Nov 2002, pp. 205-208.

[7] Z. Katona and J. Bito, "Performance of link adaptation techniques for different service classes through land mobile satellite communication channels," in Satellite and Space Communications, 2006 International Workshop on, Sept 2006, pp. 105-109.

[8] M. Bergmann, W. Gappmair, and O. Koudelka, "Parameter estimation for link adaptation on land-mobile satellite links," in Communication Systems, Networks Digital Signal Processing (CSNDSP), 2014 9th International Symposium on, July 2014, pp. 1139-1143.

[9] S. Boyd and T. Macdonald, "Satellite communications link adaptation design and interaction with tcp," in Military Communications Conference, 2005. MILCOM 2005. IEEE, Oct 2005, pp. 2042-2047 Vol. 4. 
[10] T. El Shabrawy and S. Wahed, "Adaptive modulation and coding for broadcast dvb-h systems," in Personal, Indoor and Mobile Radio Communications, 2009 IEEE 20th International Symposium on, Sept 2009, pp. 1292-1296.

[11] C. Morel, P.-D. Arapoglou, M. Angelone, and A. Ginesi, "Link adaptation strategies for next generation satellite video broadcasting: A system approach," Broadcasting, IEEE Transactions on, vol. PP, no. 99, pp. 1-1, 2015.

[12] L. Abderrahmane, D. Hamed, and M. Benyettou, "Design of an adaptive communication system for implementation on board a future algerian leo satellite," in Aerospace Conference, 2008 IEEE, March 2008, pp. 1-5.

[13] S. Cioni, R. De Gaudenzi, and R. Rinaldo, "Channel estimation and physical layer adaptation techniques for satellite networks exploiting adaptive coding and modulation," International Journal of Satellite Communications and Networking, vol. 26, no. 2, pp. 157-188, 2008. [Online]. Available: http://dx.doi.org/10.1002/sat.901

[14] C. Isheden and G. P. Fettweis, "Energy-efficient link adaptation with shadow fading," in Vehicular Technology Conference (VTC Spring), 2011 IEEE 73rd, May 2011, pp. $1-5$.

[15] "Scan testbed location on ISS," https://spaceflightsystems.grc.nasa.gov/sopo/scsmo/scan-testbed/. [Online]. Available: https://spaceflightsystems.grc.nasa.gov/sopo/scsmo/scan-testbed/

[16] S. J. A. H. W. A. Richards, Mark A. SciTech Publishing, 2010. [Online]. Available: http://app.knovel.com/hotlink/toc/id:kpPMRVIBP8/ principles-modern-radar/principles-modern-radar

[17] D. Chelmins, J. Downey, S. K. Johnson, and J. Nappier, "Unique challenges testing sdrs for space," in Aerospace Conference, 2013 IEEE, March 2013, pp. 1-9.

[18] J. G. Proakis and M. Salehi, Digital Communications, 5th ed.

[19] S. Ford, The ARRL satellite handbook. Newington, Conn: American Radio Relay League, 2008.

[20] "International space station: Solar arrays," 2013. [Online]. Available: https: //www.nasa.gov/mission_pages/station/structure/elements/solar_arrays.html

[21] G. Paynter, W. Burnside, and T. Lee, "A systematic approach to design and analysis of antennas on complex platforms," Antennas and Propagation Magazine, IEEE, vol. 43, no. 6, pp. 38-44, Dec 2001.

[22] A. D. Monk, "Antennas and arrays," in Electronics Engineer's Reference Book, F. F. Mazda, Ed., p. 49/9. 
[23] K. R. Sturley, "Noise and communication," in Electronics Engineer's Reference Book, F. F. Mazda, Ed., p. 51/5.

[24] S. Cakaj, B. Kamo, I. Enesi, and O. Shurdi, "Antenna noise temperature for low earth orbiting satellite ground stations at 1 and s band," in SPACOMM: The Third International Conference on Advances in Satellite and Space Communications, 2011.

[25] S. Haykin, Introduction to analog and digital communications. Hoboken, N.J: J. Wiley \& Sons, 2007.

[26] J. E. Allnutt, Satellite-to-ground radiowave propagation, 2nd ed. London: Institution of Engineering and Technology, 2012; vol. 54.

[27] T. Pratt, C. Bostian, and J. Allnutt, Satellite Communications, 2nd ed.

[28] S. Nanda, K. Balachandran, and S. Kumar, "Adaptation techniques in wireless packet data services," IEEE Communications Magazine, vol. 38, no. 1, pp. 54-64, Jan 2000.

[29] J. Pons and J. Dunlop, "Bit error rate based link adaptation for gsm," in Personal, Indoor and Mobile Radio Communications, 1998. The Ninth IEEE International Symposium on, vol. 3, Sep 1998, pp. 1530-1534 vol.3.

[30] T. S. Rappaport, Wireless communications: principles and practice, 2nd ed. Upper Saddle River, N.J;London;: Prentice Hall PTR, 2001.

[31] M. Lacage, M. H. Manshaei, and T. Turletti, "Ieee 802.11 rate adaptation: a practical approach," in Proceedings of the 7th ACM international symposium on Modeling, analysis and simulation of wireless and mobile systems. ACM, 2004, pp. 126-134.

[32] Z. Dou, Z. Zhao, Q. Jin, L. Zhang, Y. Shu, and O. Yang, "Energy-efficient rate adaptation for outdoor long distance wifi links," in Computer Communications Workshops (INFOCOM WKSHPS), 2011 IEEE Conference on, April 2011, pp. 271-276.

[33] W. Yang, L. Li, G. Wu, and H. Wang, "Joint relay selection and link adaptation for distributed beamforming in regenerative cooperative networks," in Information Theory and its Applications (ISITA), 2010 International Symposium on, Oct 2010, pp. 157-162.

[34] Z. Lin, E. Erkip, and M. Ghosh, "Cth15-5: Rate adaptation for cooperative systems," in Global Telecommunications Conference, 2006. GLOBECOM '06. IEEE, Nov 2006, pp. 1-5.

[35] M. R. Souryal and N. Moayeri, "Joint rate adaptation and channel-adaptive relaying in 802.11 ad hoc networks," in Military Communications Conference, 2006. MILCOM 2006. IEEE, Oct 2006, pp. 1-8. 
[36] H. Khodakarami and F. Lahouti, "Link adaptation for physical layer security over wireless fading channels," IET Communications, vol. 6, no. 3, pp. 353-362, February 2012.

[37] Y. Qiu, Y. Chen, and D. Haley, "Snr estimation and decision making using hypothesis testing in energy-efficient adaptive modulation," in Personal, Indoor, and Mobile Radio Communication (PIMRC), 2014 IEEE 25th Annual International Symposium on, Sept 2014, pp. 1949-1953.

[38] Z. Lin and A. Demir, "Link adaptation on aggregated tvws channels," in Wireless Communications and Networking Conference (WCNC), 2013 IEEE, April 2013, pp. 721-726.

[39] M. B. Pursley and T. C. Royster, "Low-complexity adaptive transmission for cognitive radios in dynamic spectrum access networks," IEEE Journal on Selected Areas in Communications, vol. 26, no. 1, pp. 83-94, Jan 2008.

[40] Q. Xu, H. Ji, and X. Li, "Goodput performance improvement in high-speed railway communication systems: A link adaptation approach," in Communications (ICC), 2014 IEEE International Conference on, June 2014, pp. 1059-1064.

[41] M. Smolnikar, T. Javornik, and M. Mohorcic, "Channel decoder assisted adaptive coding and modulation for hap communications," in Vehicular Technology Conference, $200 \%$. VTC2007-Spring. IEEE 65th, April 2007, pp. 1375-1379.

[42] K.-Y. Lin, H.-P. Lin, and M.-C. Tseng, "Link adaptation of mimo-ofdm systems using hidden markov model for high speed railway," in Communications (APCC), 2010 16th Asia-Pacific Conference on, Oct 2010, pp. 324-328.

[43] R. C. Daniels and R. W. Heath, "Link adaptation with position/motion information in vehicle-to-vehicle networks," IEEE Transactions on Wireless Communications, vol. 11, no. 2, pp. 505-509, February 2012.

[44] Y. Wang, Q. Cui, X. Tao, and M. Zhou, "Robust amc scheme against feedback delay in vehicular environment," in Communications, 2009. ICC '09. IEEE International Conference on, June 2009, pp. 1-5.

[45] A. Narula-Tam, T. Macdonald, E. Modiano, and L. Servi, "A dynamic resource allocation strategy for satellite communications," in Military Communications Conference, 2004. MILCOM 2004. 2004 IEEE, vol. 3, Oct 2004, pp. 1415-1421 Vol. 3 .

[46] J. P. Choi and V. W. S. Chan, "Predicting and adapting satellite channels with weather-induced impairments," IEEE Transactions on Aerospace and Electronic Systems, vol. 38, no. 3, pp. 779-790, Jul 2002. 
[47] A. Morello and V. Mignone, "Dvb-s2: The second generation standard for satellite broad-band services," Proceedings of the IEEE, vol. 94, no. 1, pp. 210-227, Jan 2006.

[48] G. Gardikis, N. Zotos, and A. Kourtis, "Satellite media broadcasting with adaptive coding and modulation," International Journal of Digital Multimedia Broadcasting, vol. 2009, pp. 1-10, 2009.

[49] B. P. Lathi, Modern digital and analog communication systems. New York: Oxford University Press, 2009.

[50] M. Mohammad and R. M. Buehrer, "On the impact of snr estimation error on adaptive modulation," IEEE Communications Letters, vol. 9, no. 6, pp. 490-492, Jun 2005.

[51] C. Yan, H. Wang, N. Wu, and J. Kuang, "Low complexity snr estimation for linear modulations on awgn channel," in Vehicular Technology Conference (VTC Spring), 2012 IEEE 75th, May 2012, pp. 1-5.

[52] D. R. Pauluzzi and N. C. Beaulieu, "A comparison of snr estimation techniques for the awgn channel," IEEE Transactions on Communications, vol. 48, no. 10, pp. 1681-1691, Oct 2000.

[53] M. A. Dangl and J. Lindner, "How to use a priori information of data symbols for snr estimation," Signal Processing Letters, IEEE, vol. 13, no. 11, pp. 661-664, 2006.

[54] M. Bakkali, A. Stephenne, and S. Affes, "Iterative snr estimation for mpsk modulation over awgn channels," in Vehicular Technology Conference, 2006. VTC-2006 Fall. 2006 IEEE 64th, Sept 2006, pp. 1-5.

[55] H. Xiao, Y. Q. Shi, W. Su, and J. Kosinski, "An investigation of non-data-aided snr estimation techniques for analog modulation signals," in Sarnoff Symposium, 2010 IEEE, April 2010, pp. 1-5.

[56] N. B. Carvalho and D. Schreurs, Microwave and wireless measurement techniques. New York; Cambridge, United Kingdom;: Cambridge University Press, 2013.

[57] M. D. McKinley, K. A. Remley, M. Myslinkski, J. S. Kenney, D. Schreurs, and B. Nauwelaers, "Evm calculation for broadband modulated signals," in Microwave Measurements Conference, Fall 2004. 64th ARFTG, Dec 2004, pp. 45-52.

[58] A. Doukas and G. Kalivas, "A modified evm snr estimation method for qam ofdm systems in awgn channel," in Wireless Conference 2006 - Enabling Technologies for Wireless Multimedia Communications (European Wireless), 12th European, April 2006, pp. 1-6.

[59] H. S. Park and Y. O. Park, "Snr estimation in ofdma/tdd based wibro system," in Vehicular Technology Conference, 2008. VTC 2008-Fall. IEEE 68th, Sept 2008, pp. 1-5. 
[60] D. Athanasios and K. Grigorios, "Error vector magnitude snr estimation algorithm for hiperlan/2 transceiver in awgn channel," in Telecommunications in Modern Satellite, Cable and Broadcasting Services, 2005. Tth International Conference on, vol. 2, Sept 2005, pp. 415-418 vol. 2.

[61] D.-J. Shin, W. Sung, and I.-K. Kim, "Simple snr estimation methods for qpsk modulated short bursts," in Global Telecommunications Conference, 2001. GLOBECOM '01. IEEE, vol. 6, 2001, pp. 3644-3647 vol.6.

[62] H. A. Mahmoud and H. Arslan, "Error vector magnitude to snr conversion for nondata-aided receivers," IEEE Transactions on Wireless Communications, vol. 8, no. 5, pp. 2694-2704, May 2009.

[63] R. Shafik, S. Rahman, and R. Islam, "On the extended relationships among evm, ber and snr as performance metrics," in Electrical and Computer Engineering, 2006. ICECE '06. International Conference on, Dec 2006, pp. 408-411.

[64] X. Li, Y. Chi, X. Tan, and Y. Zhao, "A data-aided evm estimator for snr utilizing zadoff-chu sequence as preamble," Procedia Engineering, vol. 29, pp. 573-578, 2012.

[65] R. Chouitem, "Evm based amc for an ofdm system," in Wireless Telecommunications Symposium (WTS), 2010, April 2010, pp. 1-5. 